\title{
Tornado Dynamics Study Using Immersed Boundary (IB) - Lattice \\ Boltzmann Method (LBM) on a 7-Cylinder Building Configuration
}

by

Rangaraj Palanisamy

B.Eng., Aeronautical Society of India, New Delhi, India, 2013

\author{
A thesis \\ presented to Ryerson University \\ in partial fulfillment of the \\ requirements for the degree of \\ Master of Applied Science \\ in the program of \\ Mechanical Engineering
}

Toronto, Ontario, Canada, 2017

(C) Rangaraj Palanisamy 2017 


\section{Author's Declaration}

I hereby declare that I am the sole author of this thesis.

I authorize Ryerson University to lend this thesis to other institutions or individuals for the purpose of scholarly research.

I further authorize Ryerson University to reproduce this thesis by photocopying or by other means, in total or in part, at the request of other institutions or individuals for the purpose of scholarly research.

I understand that my thesis may be made electronically available to the public. 


\title{
Tornado Dynamics Study Using Immersed Boundary (IB) - Lattice Boltzmann Method (LBM) on a 7-Cylinder Building Configuration
}

\author{
Master of Applied Science, 2017 \\ Rangaraj Palanisamy \\ Mechanical and Industrial Engineering \\ Ryerson University
}

\begin{abstract}
Tornadoes are disastrous, naturally occurring atmospheric phenomena; they cause fatalities; they damage properties with an exceptional combination of translational and rotational velocities. Despite many studies on tornado-structure interaction, the research papers on tornado-multi-body interactions are limited. This research studies the effects of a tornadic wind on a 7-cylinder building model at several orientations in 2-D using a powerful Immersed Boundary-Lattice Boltzmann Method (IB-LBM). The tornadic wind was simulated by a customized Rankine Combined Vortex Model (RCVM). The wind-loadings on the seven cylinders were quantified using aerodynamic force and moment coefficients. The essential flow features associated with a vortex-structure interaction was investigated in great detail by doing a case study. Then, a unique optimization procedure was utilized to detect individual safe zones for each aerodynamic coefficient. Finally, an overall safe zone for the complete 7-cylinder building model has been ascertained to be between $29^{\circ}$ and $69^{\circ}$ by analyzing the individual safe zones.
\end{abstract}




\section{Acknowledgements}

I would like to thank Dr. Jun Cao for giving me the opportunity to work on this exciting research project and for his constant encouragement and valuable guidance during this study.

This work is financially supported by Dr. Cao's Discovery Grant awarded by the National Science and Engineering Research Council (NSERC) of Canada.

I also would like to thank my colleague, Mr. Xixiong Guo, who helped me in all the aspects of my research. 


\section{Table of Contents}

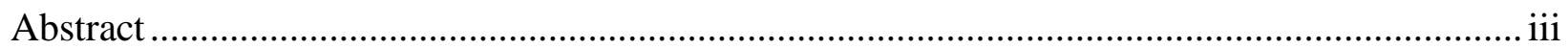

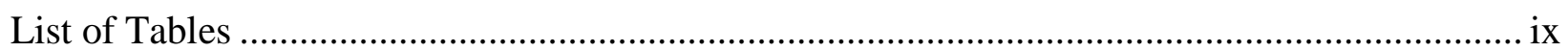

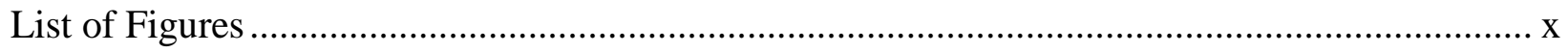

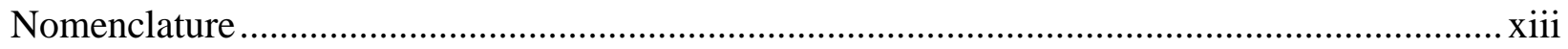

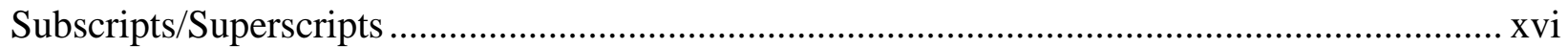

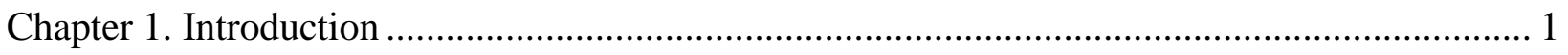

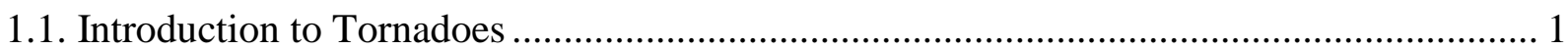

1.2. An Overview of Lattice Boltzmann Method (LBM) ............................................... 4

1.3. Background on Immersed Boundary Method (IBM) .............................................. 4

1.4. The Objectives and Significance of the Thesis ......................................................... 5

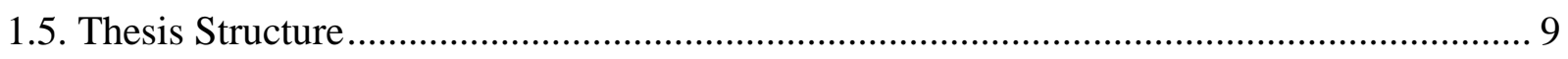

Chapter 2. Tornadic Wind Model .................................................................................... 10

2.1. Rankine - Combined Vortex Model (RCVM) ........................................................ 10

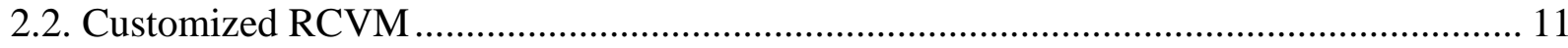

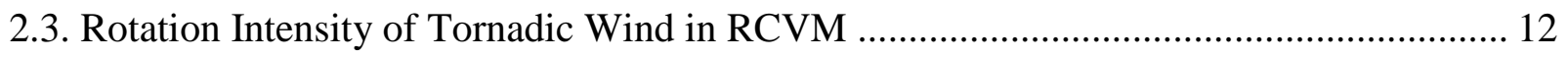

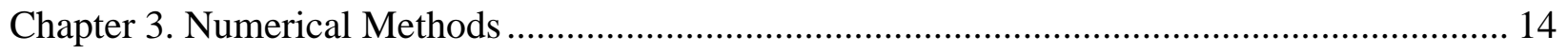

3.1. Lattice Boltzmann Method (LBM) With Multiple-Relaxation Time (MRT) Collision

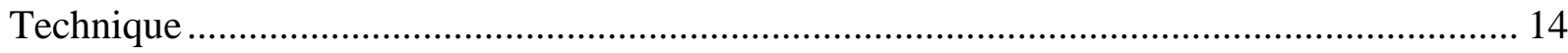


Chapter 4. Important Parameters, Numerical Experiments, and Computational Domain Setup.. 21

4.1. Definitions of Important Parameters ...................................................................... 21

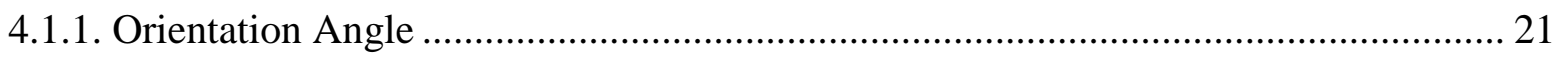

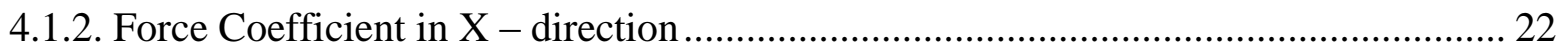

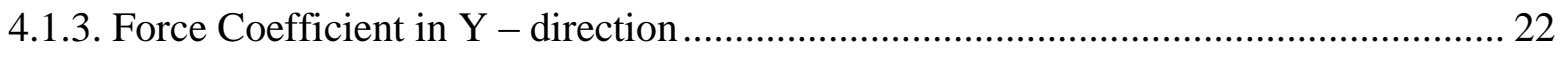

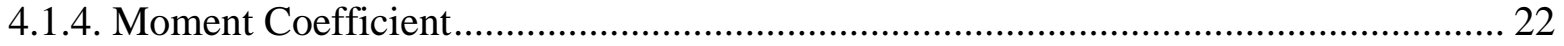

4.1.5. Resultant Coplanar Force Coefficient ............................................................. 23

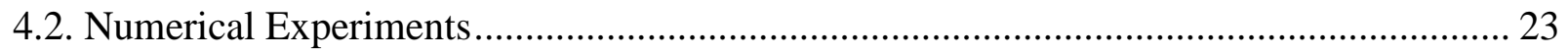

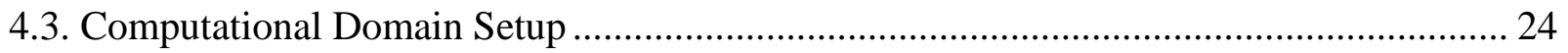

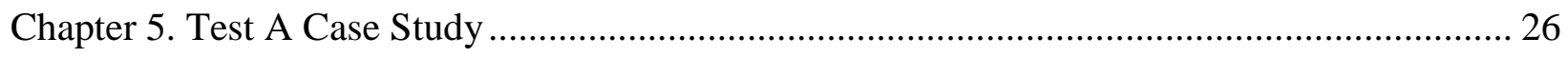

5.1. Three Stages of Tornado - Structure Interaction ................................................... 26

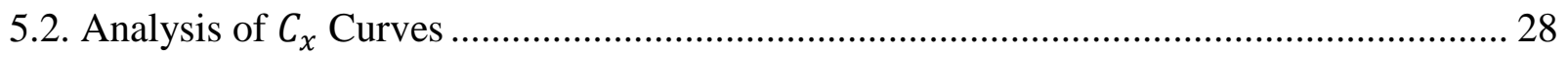

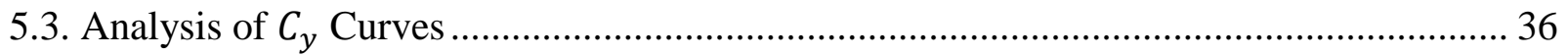

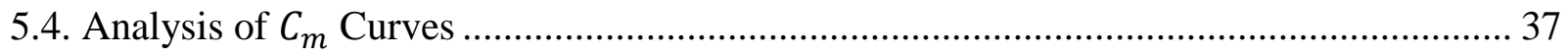

Chapter 6. Summary of the Numerical Results for Case A to G ........................................... 39

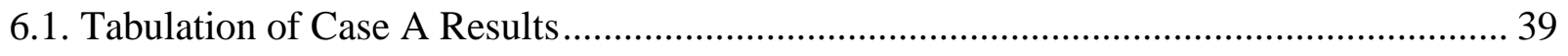

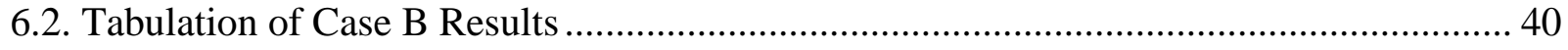

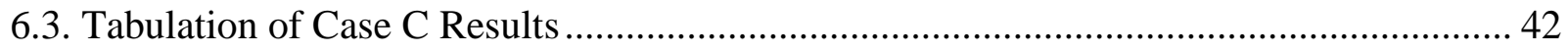

6.4. Tabulation of Case D Results ................................................................................ 45 
6.5. Tabulation of Case E Results .....

6.6. Tabulation of Case F Results ................................................................................ 50

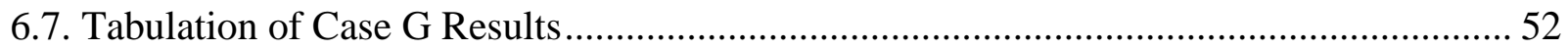

6.8. The Unluckiest or Most Affected Building ............................................................. 53

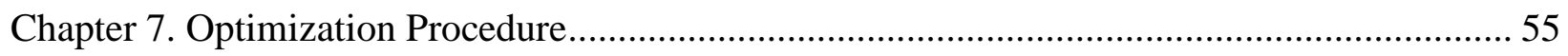

7.1. An Overview of Optimization Procedure Using Polynomial Fitting ............................ 55

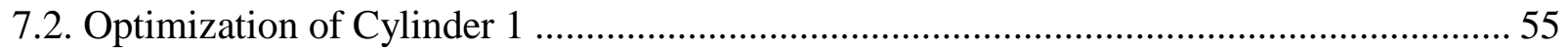

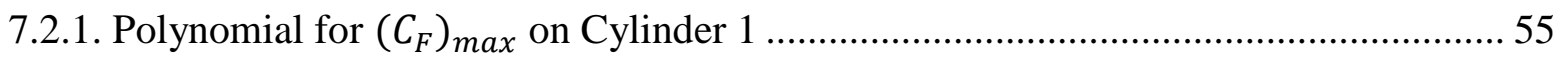

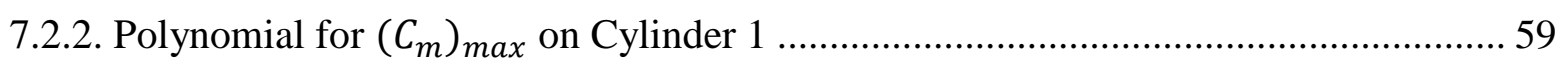

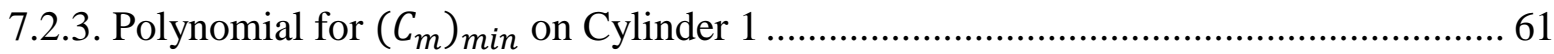

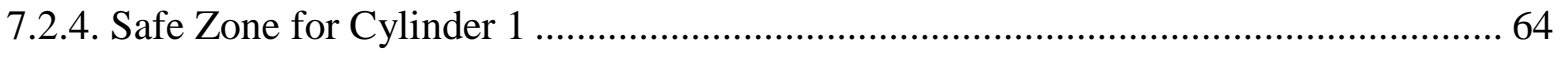

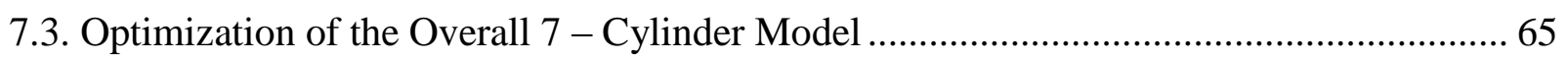

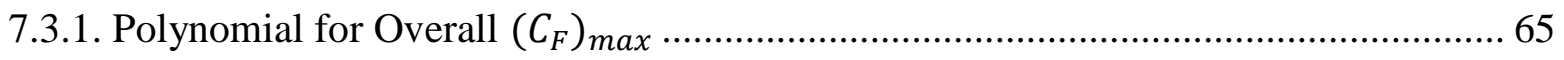

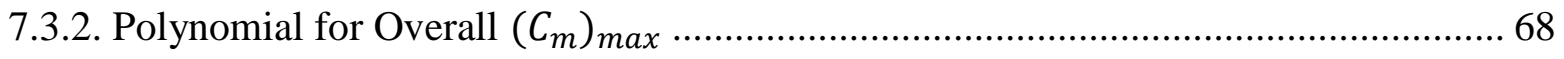

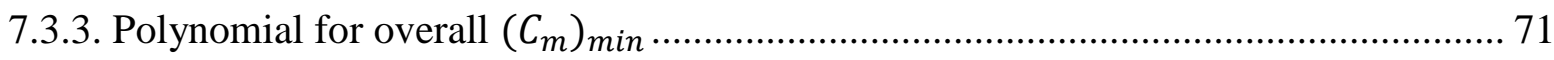

7.3.4. Overall Safe Zone for the 7 - Cylinder Model ..................................................... 73

7.4. Comparison of the Overall and Cylinder 1 Safe Zones ........................................... 75

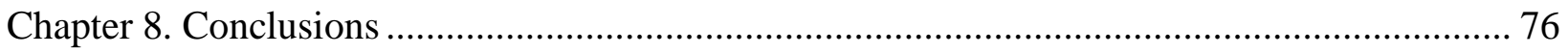

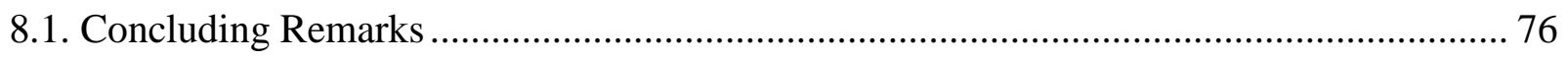

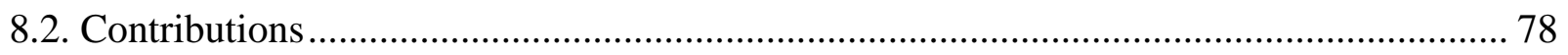


8.3. Future Prospects

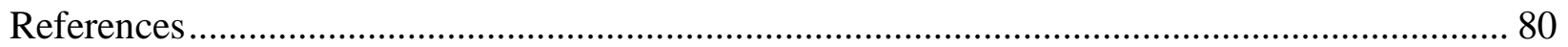




\section{List of Tables}

Table 6.1 Summary of Wind - Loading Coefficients for case A …………………………......... 39

Table 6.2 Summary of Wind - Loading Coefficients for cae B ................................................ 41

Table 6.3 Summary of Wind - Loading Coefficients for case C.................................................. 43

Table 6.4 Summary of Wind - Loading Coefficients for case D ................................................ 46

Table 6.5 Summary of Wind - Loading Coefficients for case E.................................................. 48

Table 6.6 Summary of Wind - Loading Coefficients for case F ................................................ 50

Table 6.7 Summary of Wind - Loading Coefficients for case G .............................................. 53

Table 6.8 The Most affected Cylinders of the test cases A to G ………………………............. 54

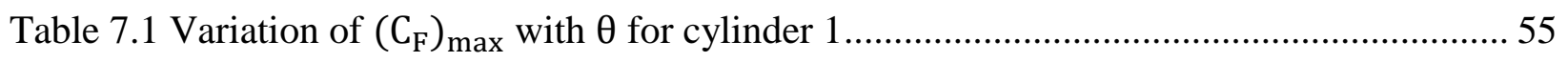

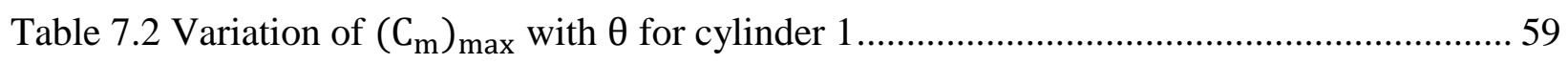

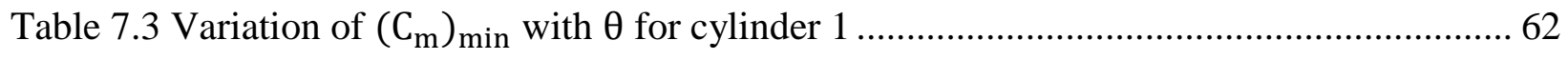

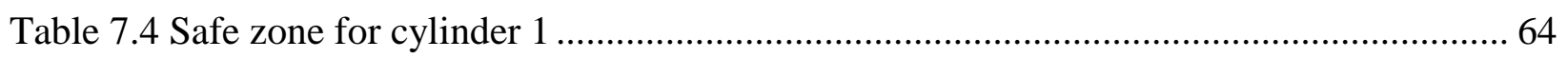

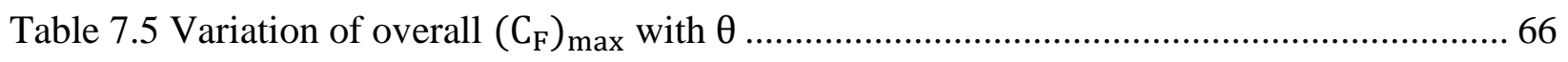

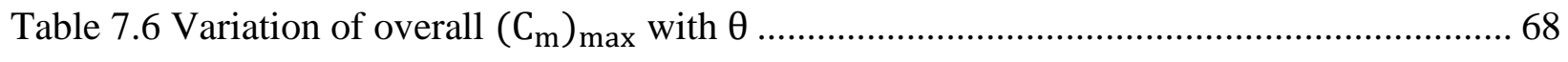

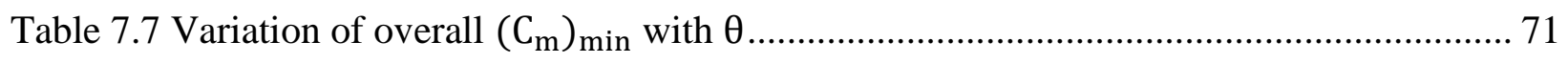

Table 7.8 Overall safe zone for the complete 7 -cylinder model................................................ 74 


\section{List of Figures}

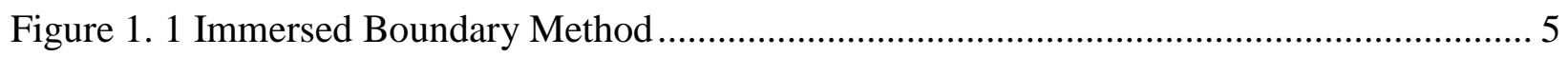

Figure 1. 2 Tornado damage in Louisville, Mississippi on April 29, 2014. Credit: Mississippi

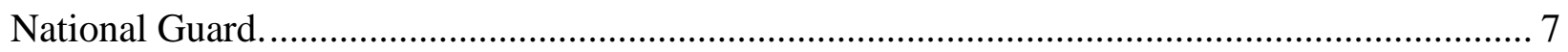

Figure 1. 3 Illustration of 7-cylinder arrangement and tornado incoming direction ..................... 7

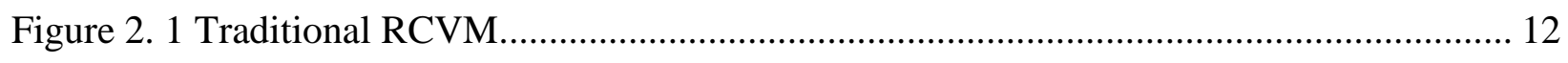

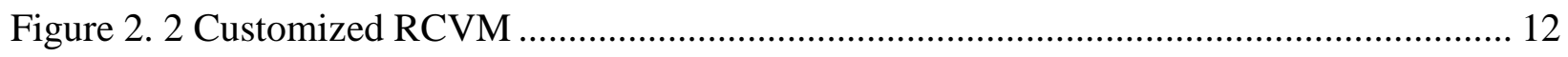

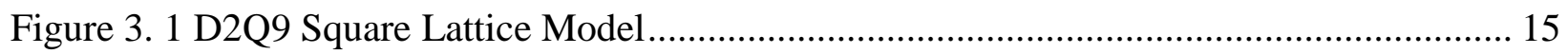

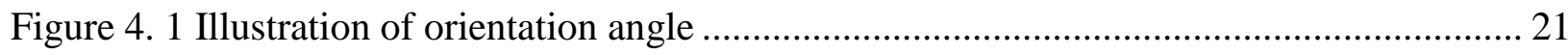

Figure 4. 2 Illustration of 7 test cases A to $\mathrm{G}$ and their orientation angles.................................. 24

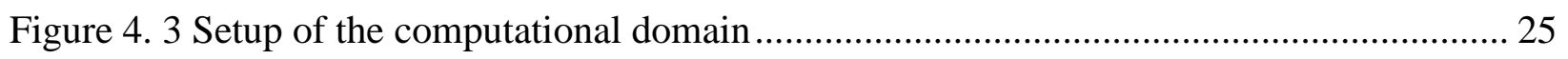

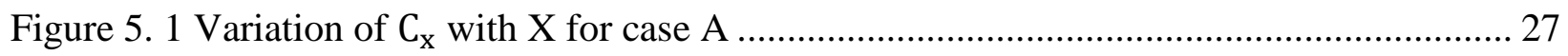

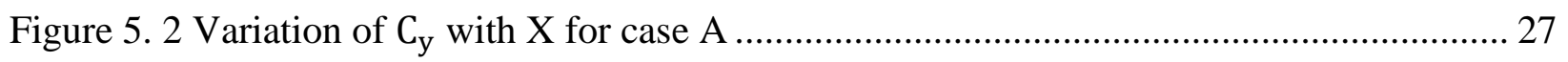

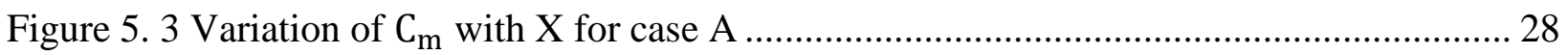

Figure 5. 4 Velocity magnitude contour corresponding to point (1) of figure 5.1 ....................... 30

Figure 5. 5 Velocity magnitude contour corresponding to points 1) (2) of figure 5.12) (b) of figure

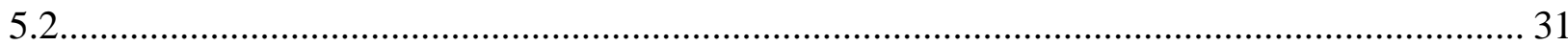

Figure 5. 6 Velocity magnitude contour corresponding to points 1) (3) of figure 5.12) (5)of figure

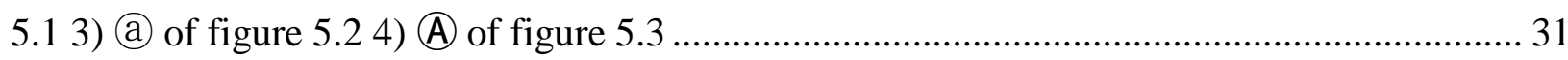

Figure 5. 7 Velocity magnitude contour corresponding to point (4) of figure 5.1 ........................ 32

Figure 5. 8 Velocity magnitude contour corresponding to points 1) (6) of figure 5.12) (e) of figure

5.2 
Figure 5. 9 Velocity magnitude contour corresponding to points 1) (7) of figure 5.12) (d) of figure $5.23)$ (B) of figure 5.3 34

Figure 5. 10 Velocity magnitude contour corresponding to points 1) (8) of figure 5.12) (C) of figure 5.3. 35

Figure 5. 11 Velocity magnitude contour corresponding to points 1) (9) of figure 5.12) (C) of figure

5.2 35

Figure 6. 1 Velocity magnitude contour at $\left(\mathrm{C}_{\mathrm{F}}\right)_{\max }$ on cylinder 1 for case $\mathrm{A}$ 41

Figure 6. 2 Velocity magnitude contour at $\left(\mathrm{C}_{\mathrm{F}}\right)_{\max }$ on cylinder 1 for case $\mathrm{B}$. 42

Figure 6. 3 Velocity magnitude contour at 1$)\left(C_{m}\right)_{\min }$ on cylinder 2 for case $\left.B 2\right)\left(C_{F}\right)_{\max }$ on cylinder 1 for case $\mathrm{C}$ 44

Figure 6. 4 Velocity magnitude contour at $\left(\mathrm{C}_{\mathrm{m}}\right)_{\min }$ on cylinder 2 for case $\mathrm{C}$ 45

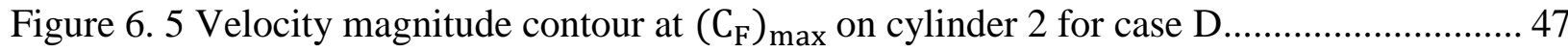

Figure 6. 6 Velocity magnitude contour at $\left(\mathrm{C}_{\mathrm{m}}\right)_{\min }$ on cylinder 3 for case $\mathrm{D}$........................ 49

Figure 6.7 Velocity magnitude contour at $\left(C_{m}\right)_{\min }$ on cylinder 7 for case E....................... 49

Figure 6.8 Velocity magnitude contour at $\left(\mathrm{C}_{\mathrm{F}}\right)_{\max }$ on cylinder 3 for case E ........................ 51

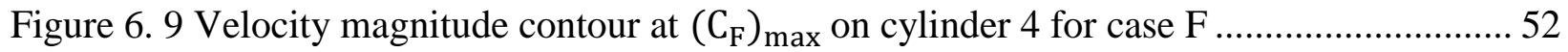

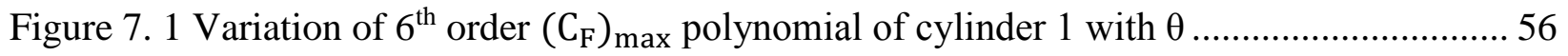

Figure 7. 2 Variation of $7^{\text {th }}$ order $\left(C_{F}\right)_{\text {max }}$ polynomial of cylinder 1 with $\theta \ldots \ldots \ldots \ldots \ldots \ldots \ldots \ldots \ldots . . . . . \ldots \ldots$

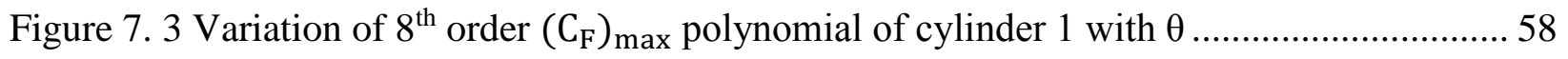

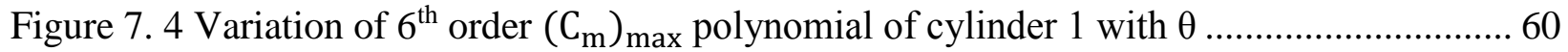

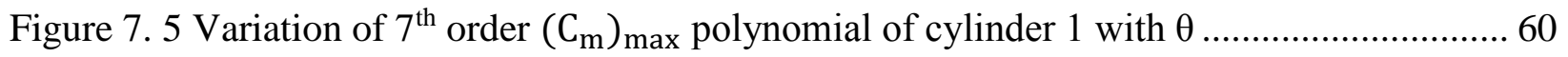

Figure 7. 6 Variation of $8^{\text {th }}$ order $\left(C_{m}\right)_{\max }$ polynomial of cylinder 1 with $\theta \ldots \ldots \ldots \ldots \ldots \ldots \ldots \ldots \ldots . \ldots \ldots \ldots$

Figure 7. 7 Variation of $6^{\text {th }}$ order $\left(C_{m}\right)_{\text {min }}$ polynomial of cylinder 1 with $\theta \ldots \ldots \ldots \ldots \ldots \ldots \ldots \ldots \ldots . . . . \ldots 3$ 
Figure 7.8 Variation of $7^{\text {th }}$ order $\left(C_{m}\right)_{\min }$ polynomial of cylinder 1 with $\theta$ 63

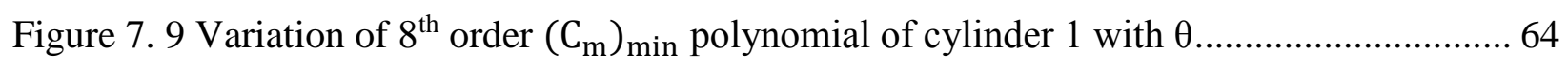

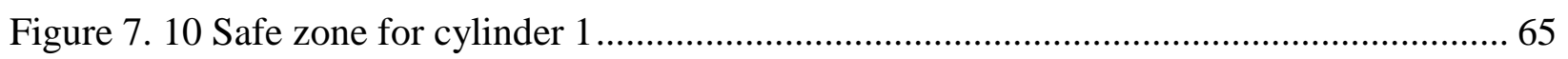

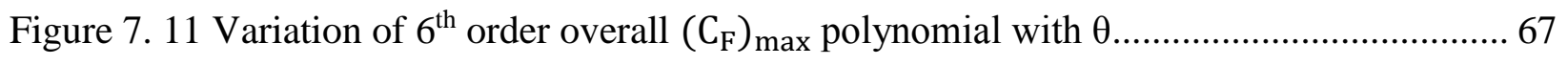

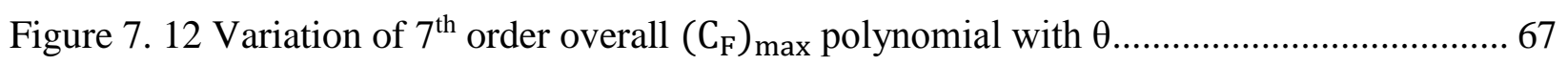

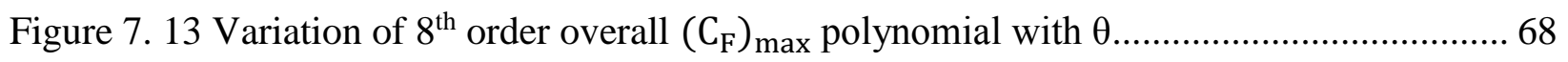

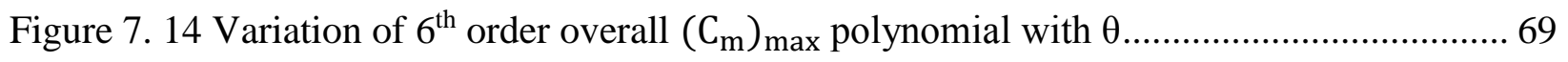

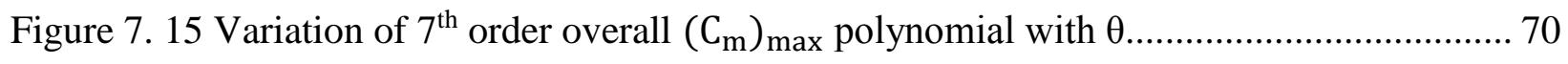

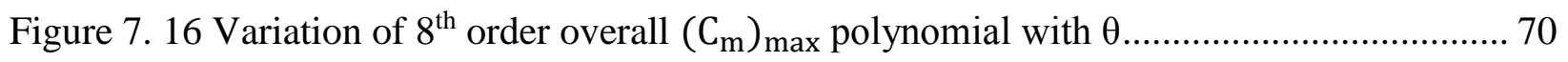

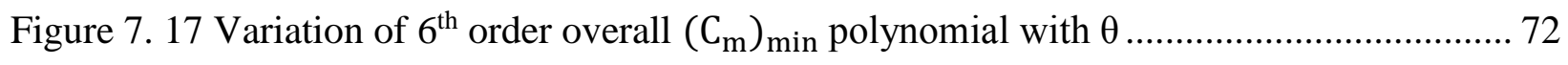

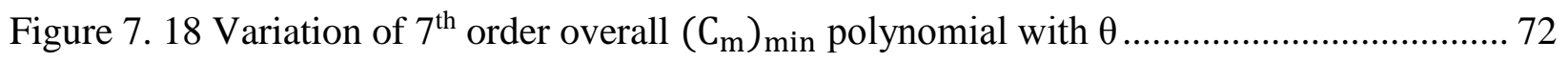

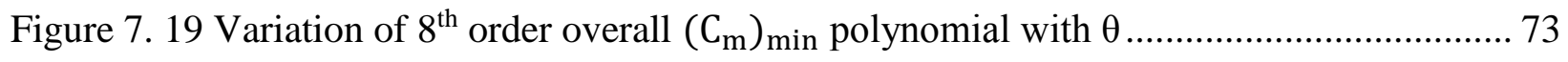

Figure 7. 20 Overall safe zone for the complete 7 -cylinder model ..................................... 74 


\section{Nomenclature}

\section{In English}

$C_{x} \quad$ Force coefficient in the X-direction

$C_{y} \quad$ Force coefficient in the Y-direction

$C_{m} \quad$ Moment coefficient

$C_{F} \quad$ Resultant planar force coefficient

$F_{x} \quad$ Force in the $\mathrm{X}$-direction

$F_{y} \quad$ Force in the Y-direction

M Moment

$\theta \quad$ Orientation angle

$\boldsymbol{V}_{\boldsymbol{t}} \quad$ Translation velocity vector

$V_{t x} \quad$ Translation velocity component in the X-direction

$V_{t y} \quad$ Translation velocity component in the Y-direction

$\boldsymbol{V}_{\boldsymbol{\gamma}} \quad$ Tangential velocity vector

V Resultant or total velocity vector

$r_{c} \quad$ Core radius of the tornado

c $\quad$ Lattice speed

$c_{s} \quad$ Speed of sound in lattice

$c_{i} \quad$ Particle distribution function

f Distribution function vector

$f^{e q} \quad$ Equilibrium distribution function vector

$\boldsymbol{R} \quad$ Moment vector 


$\begin{array}{ll}\boldsymbol{R}^{e q} & \text { Equilibrium moment vector } \\ \boldsymbol{M} & \text { Transformation matrix } \\ \boldsymbol{S} & \text { Diagonal relaxation matrix } \\ w_{i} & \text { Weighing factor } \\ p & \text { Pressure of the fluid } \\ u_{x} & \text { Velocity component of the fluid in the X-direction } \\ u_{y} & \text { Velocity component of the fluid in the Y-direction } \\ \boldsymbol{u} & \text { Resultant velocity vector of the fluid } \\ \mathrm{D} & \text { Diameter of the cylindrical buildings } \\ \boldsymbol{X}_{\boldsymbol{B}}^{l} & \text { Location vector of the Lagrangian points } \\ \boldsymbol{U}_{\boldsymbol{B}}^{l} & \text { Velocity vector at the Lagrangian points } \\ f_{R S T} & \text { Restoring force at the Eulerian points } \\ F_{R S T} & \text { Restoring force at the Lagrangian points } \\ \boldsymbol{U}_{\boldsymbol{I M S}} & \text { Velocity of the immersed body } \\ s & \text { Parametric coordinate of the Lagrangian points }\end{array}$

\section{In Greek}

$\begin{array}{ll}\omega & \text { Angular velocity of the tornado } \\ \beta & \text { Rotation intensity of the tornado } \\ \gamma & \text { Angle between radial line and the horizontal in the tornado } \\ \zeta & \text { Kinematic viscosity of the fluid } \\ \tau & \text { Relaxation rate }\end{array}$


$\Gamma \quad$ Boundary of the immersed body

$\mathbf{E} \quad$ Eulerian points in the flow field

$\rho \quad$ Density of the fluid

$\Delta s_{l} \quad$ Arc length of the boundary segment

$\delta x \quad$ Lattice spacing in the $\mathrm{X}$-direction

Sy Lattice spacing in the Y-direction 


\section{Subscripts/Superscripts}

( $)_{B}^{l} \quad$ At the Lagrangian point and at the boundary of the immersed object

( ) $)_{R S T} \quad$ Restoring force

( ) $)_{\max } \quad$ Maximum coefficient

( ) $)_{\min } \quad$ Minimum coefficient

()$_{i j} \quad$ At the Eulerian point $(\mathrm{i}, \mathrm{j})$ 


\section{Chapter 1 - Introduction}

\subsection{Introduction to Tornadoes}

Tornadoes are inherently destructive; short-lived; capable of producing unimaginable damage to both properties and lives. It is a type of vortex airflow that combines translational, vertical and rotational velocities. This complex combination makes the flow unique and more lethal compared to straight-line flows. Moreover, the combination is also responsible for tornado's unpredictable and highly precipitous nature in terms of speed and direction.

Over the last two to three decades, the improved warning systems and preparedness have reduced the casualties and injuries caused by tornadoes to a marked extent. But the number of tornadoes reported every year around the globe have been increasing at an alarming rate. For instance, an average of 1253 tornadoes occur every year in the United States alone as reported by National Climatic Data Center[1]. Therefore, a thorough understanding of the dynamics of tornado and it's interaction with the structures are essential not only to reduce the destruction, but also to construct the buildings to be inordinately wind-resistant. There are two ways to do so; either to create the tornado-structure interaction in the laboratory or to simulate it numerically using computational techniques.

Construction of a tornadic wind with all its basic elements in the laboratory involves numerous onerous tasks. In particular, imparting a high translation velocity to the tornado in the laboratory poses a great risk too. However, many researchers have tried to produce the tornadic wind physically and succeeded to some degree. Ward[2] first designed a tornado model using Euler's Momentum theorem, which was capable of producing vortices of different sizes as well as multiple vortices. But the main drawback with the model was that it was not equipped to provide 
translational velocity to the vortex. Wan and Chang[3] made use of a 3-D velocity probe and created comprehensive mappings of the velocity fields for two different swirl ratios. Subsequently, Church et al.[4] configured the transition points at which the single-cell tornado transformed into double-cell structures. In addition, they demonstrated those points to be functions of swirl ratio and Reynolds number. Davies-Jones[5] investigated the dependency of core radius on swirl ratio and showed that, for a given circulation and updraft radius of the tornado simulator, narrow vortices require high volume flow rates for its sustainability. During the same period, Diamond and Wilkins[6] modified tornado simulator to impart translation and demonstrated that the translation locally increases swirl ratio and thereby produces a bigger core compared to stationary vortex. Mitsuta and Monji[7] modified the simulator to induce circulation and found out that the maximum horizontal velocity occurs near the ground's surface and the height of maximum velocity is independent of swirl ratio. Matsui and Tamura[8] showed that the floor roughness plays an effective role on tornadoes having lower swirl ratios than the higher ones. Zhang and Sarkar[9] investigated the vortex structure near the ground surface and established the dominance of tangential velocity component over the radial component by three times. Recently, Tari et al.[10] showed that the core radius, radial, and tangential components increase for higher swirl ratios. While the physical simulations generate productive results, they have a myriad of reservations too; they are laborious, expensive; difficult to redesign; and they may become futile. Thus, the simulation of tornadoes using numerical techniques has emerged as a prominent alternative to study the vortex structures.

Several researches have been performed by many scholars on the numerical simulation of tornadoes as well as tornado-structure interactions. Harlow and Stein[11] numerically simulated vortices of various intensities and investigated many flow related parameters. Rotunno[12]-[13] 
numerically simulated Ward's tornado simulator and showed that the core radius is independent of the Reynolds number at higher Reynolds numbers. Moreover, his numerical surface pressure patterns are in accord with the Ward's experimental results. Nolan and Ferrell[14] explored the structure and dynamics of axisymmetric vortices and demonstrated that the vortex Reynolds number controls the structure and maximum wind speed of the tornado. Lewellen and Lewellen[15]-[16] numerically simulated a 3-D tornado and studied the intensity of the vortex near the ground surface. Hangan and Kim[17] analyzed the dependency of flow dynamics on swirl ratio and its relation with the Fujita scale. Ishihara et al.[18] used the numerical Large Eddy Simulation (LES) turbulence model and simulated one-celled and two-celled vortices with two different swirl ratios. They further showed that the peak vertical velocity occurs at the center for one-celled vortex, whereas it appears near the radius of maximum tangential velocity for two-celled vortex. Natarajan and Hangan[19] also used LES model to study the effects of surface roughness and translation on the mean tangential velocity of the tornadic wind for different swirl ratios. They reported that the translation reduces the maximum mean tangential velocity for lower swirl ratios, whereas it faintly increases for higher swirl ratios. Moreover, the mean tangential velocity was also found to decrease for all swirl ratios because of surface roughness.

In addition to the aforementioned numerical methods, there are plenty of other numerical techniques available for the simulation of tornadic wind in the conventional CFD approaches of solving the Navier-Stokes equations. But, the Immersed Boundary-Lattice Boltzmann Method (IBLBM) framework has been a revelation in the fluid flow problems in the recent years. In this paper, the IB-LBM framework along with Rankine Combined Vortex Model (RCVM) is utilized to simulate the vortex-structure interaction and are introduced in the following passages. 


\subsection{An Overview of Lattice Boltzmann Method (LBM)}

Lattice Boltzmann method, shortly known as LBM, has been demonstrated as an effective and efficient alternative method to the orthodox computational approach using Navier-Stokes equations to simulate fluid flow problems[20]. LBM, having its roots in the kinetic theory, examines the dynamics of fictitious particles using a density distribution function and it is applied on a Cartesian mesh. The standard LBM involves collision and streaming process for every time step and the variables such as density and velocity are calculated using the distribution function from the laws of conservation of mass and momentum. The cardinal advantages of LBM are simplicity, easy execution, and innate parallel computation capacity. Because of these advantages, LBM has drawn lot of applications and Succi et al.[21] presented a variety of applications from laminar to fully turbulent flows in 2D and 3D using Lattice Boltzmann equations and concluded that the LBM is an adequate computational tool to analyze fluid flows on various flow regimes.

\subsection{Background on Immersed Boundary Method (IBM)}

The immersed boundary method (IBM), as the name suggests, is used to simulate the moving boundaries of the objects in a fluid flow and it is first proposed by Peskin[22] in the 1970s. Being a non-boundary conforming method, the IBM governing equations are solved on a fixed Cartesian grid and the boundary points do not coincide with the grid points. Figure 1.1 shows the schematic of the immersed boundary method. As illustrated in the Figure 1.1, the flow field is represented by a set of Eulerian or fixed Cartesian points and the boundary of the immersed object is represented by a set of Lagrangian points. This idea of representing the flow field using fixed Cartesian points gives rise to the integration of IB and LBM techniques, called IB-LBM. Feng and Michaelides[23] first successfully merged the IB method with the LBM and substantiated its advantages on the simulation of $2 \mathrm{D}$ and $3 \mathrm{D}$ particulate flows[24]. IBM models the physical boundary to be 
deformable and the restoring forces on the boundary, which is created while being distorted by the fluid flow over and around it, are distributed on the affected Eulerian points as a body force by using a discrete delta function. The major issue associated with the IBM is the computation of restoring force. There are so many ways to calculate the restoring force such as penalty method[22], direct forcing method[25], and momentum exchange method[26]. But the major drawback with all the three methods is that the restoring force is pre-calculated and this precalculated restoring force does not guarantee the satisfaction of no-slip condition at the boundary. Therefore, a more powerful and more accurate variation of IB-LBM is proposed by Jie Wu et al.[27] , in which the restoring force is not pre-calculated and is determined by enforcing the noslip on the boundary and no flow penetration is observed in the results. In the field of fluid-solid interaction, this innovative IB-LBM finds lot of applications[28]-[32].

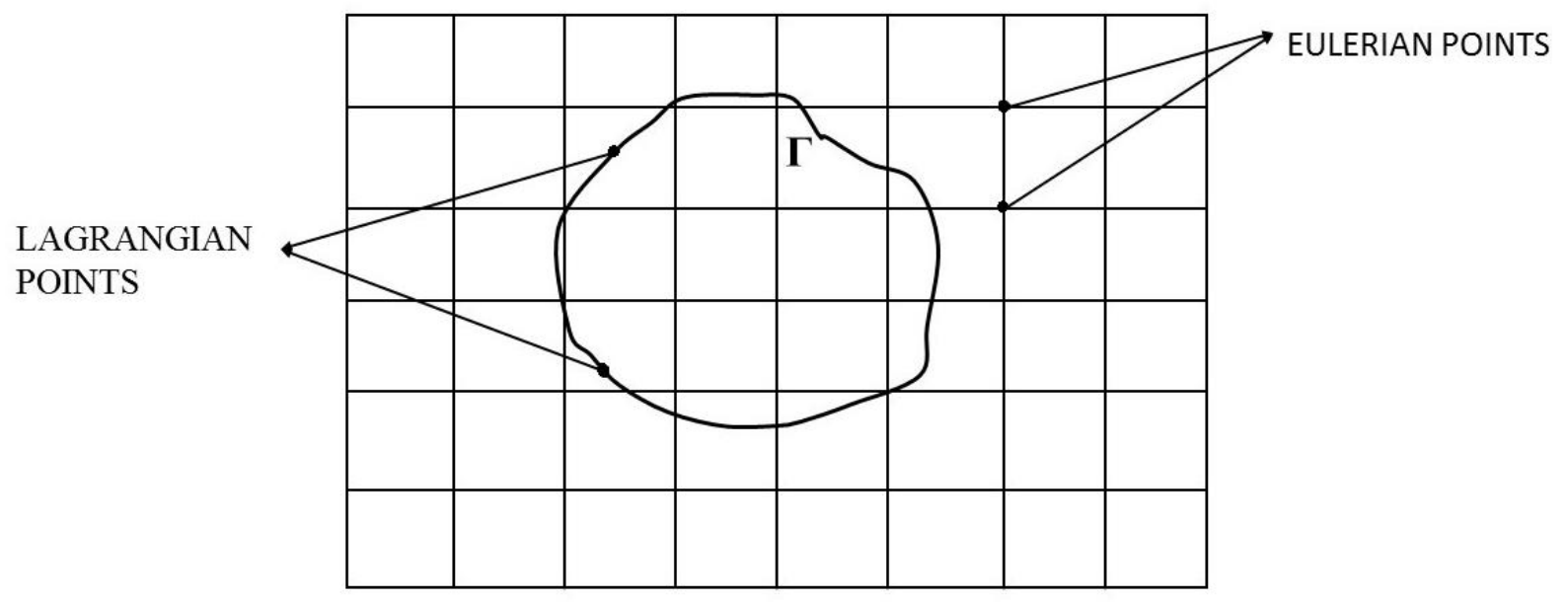

Figure 1.1 Immersed Boundary Method

\subsection{The Objectives and Significance of the Thesis}

When a tornado crosses a group of buildings, the severity of tornado is not the same on all the buildings; it is harsh on some buildings and gentle on some buildings. Figure 1.2 shows an after- 
tornado picture of a cluster of buildings in which some buildings are completely destroyed and some are slightly affected. This dual nature, cruelty and tenderness, of the tornadoes is attributed to the orientation of the buildings with respect to the direction of the path of tornadoes. But, predicting the path of tornadoes accurately is highly impractical. However, analysis of the tornadoprone places would reveal the history of the direction of the path of tornadoes. This paper focuses on identifying the cylinders which are affected the highest and the lowest in the group of a seven cylinder building model that has the cylinders arranged in a semicircular pattern, for a given tornado direction and orientation of the buildings, using the novel IB-LBM framework in two dimensions. A lot of researchers have investigated the wind-loadings on a single structure either using 2-D or 3-D numerical techniques[33]-[35]. Recently, Guo et al.[36] examined the tornado wind-loadings on a two-cylinder model with the two cylinders arranged in horizontal, vertical and 45 degree orientations using the IB-LBM framework. What makes the current research different and unique from others is that it deals with wind-loadings on an arrangement with more than two structures. The tornado-flow model is simulated using a modified Rankine-Combined Vortex Method (RCVM), which is explained in the subsequent sections. Figure 1.3 shows the arrangement of the seven cylinder building model, with the cylinders numbered from 1 to 7 , and the incoming initial flow direction of the tornado-like airflow towards the model. As it can be seen, the cylinders are arranged in a semi-circular pattern, which is the essential case study of this research. There are many reasons behind taking up the semi-circular model to do the case study; the cylinders in the middle are exposed to the perturbed core of the tornado, in comparison with the straight line model, since the tornado core reaches the cylinders at the extreme ends before it reaches the middle cylinders; the orientation can be varied in order to find ways to reduce the wind-loadings on the buildings, which is not possible with the straight-line model. The basic idea of this study is multi- 
fold: a) to understand the tornado dynamics using a 7-cylinder building model, for a given tornado direction, b) to determine the maximum affected cylinder in the model, which needs to be built stronger to withstand the brutality of the tornado, c) to determine a safe zone for the maximum affected cylinder in the model, d) to assess the individual safe zones for each aerodynamic coefficients, and e) to determine an overall safe zone for the complete 7-cylinder model with the help of the individual safe zones of the aerodynamic coefficients.

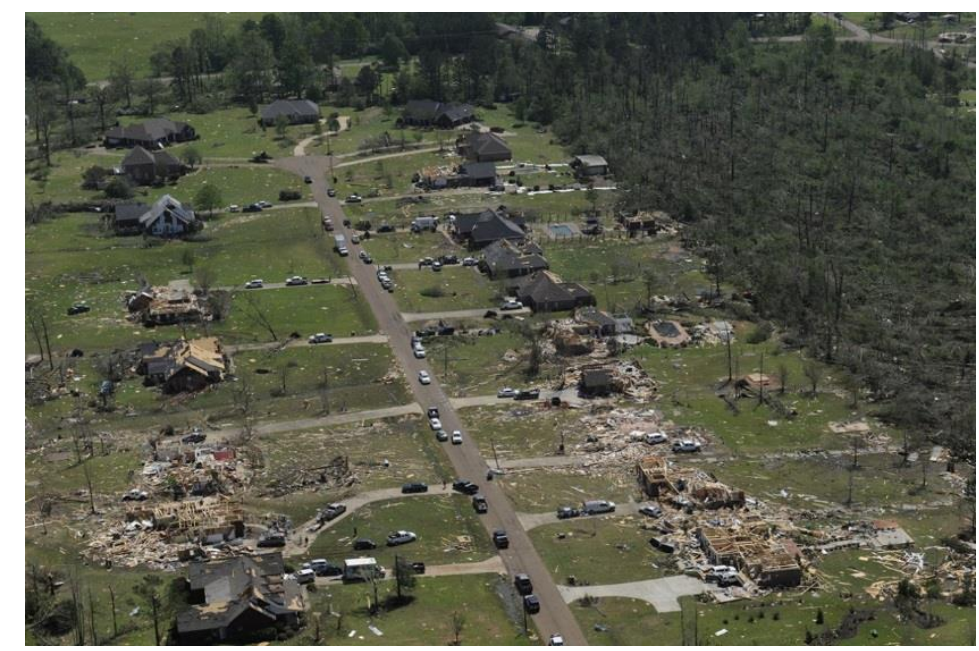

Figure 1.2 Tornado damage in Louisville, Mississippi on April 29, 2014. Credit: Mississippi National Guard.

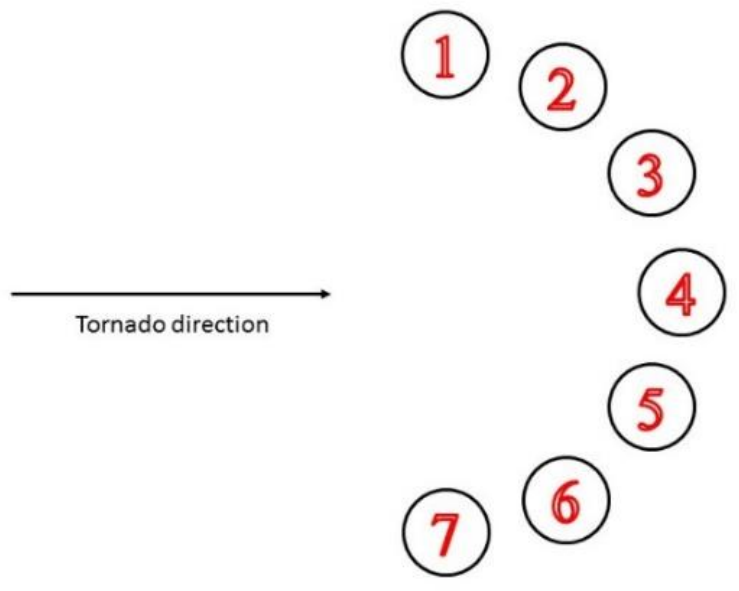

Figure 1.3 Illustration of 7-cylinder arrangement and tornado incoming direction 
The frequency of tornadoes are high in certain places and there is a high possibility that the tornadoes may reoccur in the same location and in the same direction. Historically, there are many places that are hit by the tornadoes coming essentially from one direction. This study intends to reduce the damages caused by suggesting some building orientations that result in lower wind loading coefficients, provided the tornadoes reoccur in the same direction. For this purpose, this paper places a seven building arrangement in a tornado at seven different orientation angles and these seven test cases are named as A to G, which will be explained later in Chapter 4.

The wind loading coefficients obtained from the seven test cases have been used to optimize the building orientation for a given tornado direction using an optimization procedure which is explained in Chapter 7 in great detail. The optimization has been performed on the basis of two wind loading coefficients, resultant planar force coefficient, $C_{F}$, and moment coefficient, $C_{m}$, and they are strong functions of the orientation angle. The optimization procedure involves fitting polynomials of higher orders as a function of orientation angle to each of the coefficients, $\left(C_{F}\right)_{\max }$, $\left(C_{m}\right)_{\max }$, and $\left(C_{m}\right)_{\min }$. The polynomials have been investigated to find the safe zones, a range of orientation angles at which the aforementioned three coefficients achieve lower magnitudes. For demonstrating the safe zone, the polynomials are plotted on a graph and eventually becoming evidence to the higher wind-loading coefficients in the non-safe zones. With the help of the graphs, safe zones can be identified easily and better orientations can be found which would result in the least damage caused by the tornado.

The first half of the study would explain the test case A, as illustrated in Figure 1.3 and all the flow features associated with the tornado-structure interaction with the help of flow visualization pictures at various times steps. The wind loadings on the buildings have been quantified with the help of coefficient curves and the coefficient values of all the seven cylinders involved have been 
tabulated, which in turn identified the cylinder 1 to be the most affected one. The next part of the study is solely intended to optimize the building orientation and to identify and suggest the safe zones with better orientations which suffer the least. This study deals with a practical tornadostructure interaction model for a wind engineering application.

\subsection{Thesis Structure}

The rest of the thesis is unrolled in the following pattern: Chapter 2 presents the standard RCVM and its customization used in this study followed by a summary of the numerical technique IB-LBM in Chapter 3. Chapter 4 defines the important parameters used in the study to quantify the wind-loadings on the building model. It also explains the seven cylinder building arrangement as well as the orientation angles used for the test cases A to G. Further, it gives the details used to set up the computational domain. Chapter 5 presents and explains the series of numerical results obtained for case A and analyses the wind-loadings on the individual cylinders. Chapter 6 is completely dedicated to tabulate the summary of wind-loadings for all the seven test cases. Chapter 7 carries out the optimization procedure used to find the safe zones and discusses the crucial results of the procedure. Finally, remarks are mentioned in Chapter 8, addressing the conclusions and possible extensions of the study. 


\section{Chapter 2 - Tornadic Wind Model}

\subsection{Rankine - Combined Vortex Model (RCVM)}

The Rankine Combined Vortex Model (RCVM) is the most straightforward and uncomplicated model to simulate a tornado-like airflow domain. In RCVM, the velocity profile is broken down into two velocity components, $V_{t}$, the translation velocity component, and $V_{\gamma}$, the tangential velocity component. The RCVM tornado flow domain is composed of two regions, the forced-vortex and the free-vortex. In the forced-vortex region, $V_{\gamma}$ increases linearly with radius, and in the free-vortex region, $V_{\gamma}$ decreases inversely with the radius. The radius of the forcedvortex region, $r_{c}$, is usually considered to be the core region of the tornado and the free-vortex region is considered to be the outer region of the tornado. The mathematical expressions for the RCVM can be formulated as given below in Eq. (2.1):

$$
V_{\gamma}= \begin{cases}r \omega, & r \leq r_{c} \\ \frac{r_{c}^{2} \omega}{r}, & r>r_{c}\end{cases}
$$

where $r$ is the radial distance from the center of the tornado and $\omega$ is the constant angular velocity.

Even though this conventional RCVM has a specific advantage of satisfying Navier-Stokes equations[33] in both the regions, it has a limitation that it can only be applied to a 2-D obstacle free domain. But in this numerical model, the flow domain is initialized using the traditional RCVM through some modifications. In this RCVM model, at any time, $t$, the resultant anticlockwise velocity at any point $(x, y)$ is given by:

$$
V=V_{t}+V_{\gamma}
$$


The translational velocity is given by:

$$
\boldsymbol{V}_{\boldsymbol{t}}=V_{t x} \boldsymbol{i}+V_{t y} \boldsymbol{j}
$$

The tangential velocity is given by:

$$
\boldsymbol{V}_{\gamma}=-V_{\gamma} \sin \gamma \boldsymbol{i}+V_{\gamma} \cos \gamma \boldsymbol{j}
$$

where $\gamma$ is given by $\gamma=\tan ^{-1}\left[\frac{y-y_{c}(t)}{x-x_{c}(t)}\right]$, the angle between the line passing through the point $(\mathrm{x}, \mathrm{y})$ and the horizontal line and $x_{c}(t)=x_{c 0}+V_{t x} t$ and $y_{c}(t)=y_{c 0}+V_{t y} t$, where $\left(x_{c 0}, y_{c 0}\right)$ represents the center of the tornado. Eq. (2.2), (2.3), and (2.4) reveal the time-dependent nature of the velocity of the tornado in the RCVM model. In short, $\boldsymbol{V}=\boldsymbol{V}(x, y, t)$, since $\gamma=\gamma(x, y, t)$.

\subsection{Customized RCVM}

The time-dependent nature of the velocity in the customary RCVM model is basically due to its translational component. The translational component requires updating the boundary conditions every time step, which is very tedious and complicated. To overcome this problem, the "relative motion" concept is used here, in which the translation component is detached from the tornado and is re-attached to the buildings thereby creating a "virtual translation" of the buildings towards the tornado. The velocity of the buildings is equal to the translation component of the tornado in magnitude but opposite in direction. The center of the tornado is frozen at its center $\left(x_{c 0}, y_{c 0}\right)$ and the translation velocity components, $V_{t x}$ and $V_{t y}$ are taken to be zero. Therefore the different terms become $x_{c t}=x_{c o}, y_{c t}=y_{c 0}, \gamma=\tan ^{-1}\left[\frac{y-y_{c 0}}{x-x_{c 0}}\right]$, and $\boldsymbol{V}_{\boldsymbol{t}}=\mathbf{0}$. In short, $\boldsymbol{V}=$ $\boldsymbol{V}(x, y)$, since $\gamma=\gamma(x, y)$. The "virtual translation" of the building is modelled by the immersed boundary approach which will be explained in the next chapter. The time-independent nature of this new, innovative, and customized RCVM is simple and more efficient. Figure 2.1 illustrates 
the nature of traditional RCVM with both the translation and rotation velocity components attached to the tornado, whereas Figure 2.2 shows the essence of the customization process with only the rotation velocity component attached to the tornadic wind.

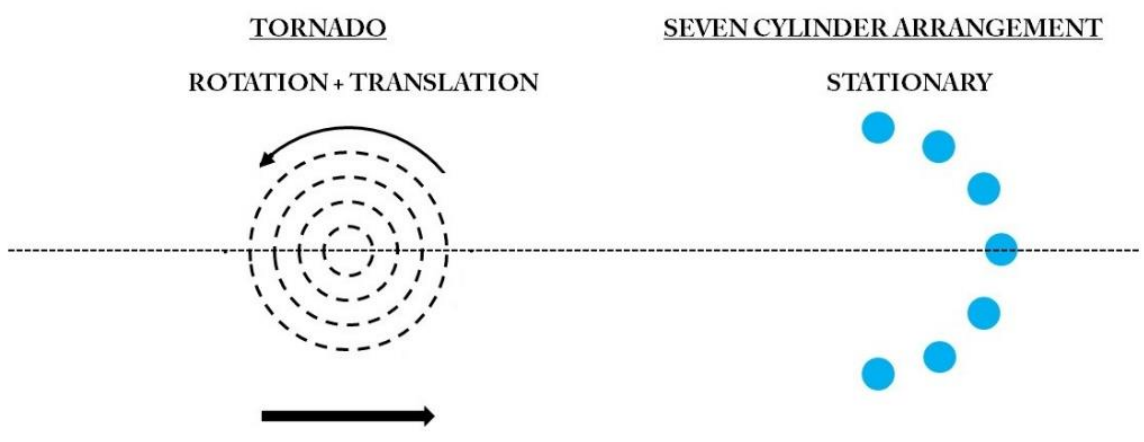

Figure 2.1 Traditional RCVM

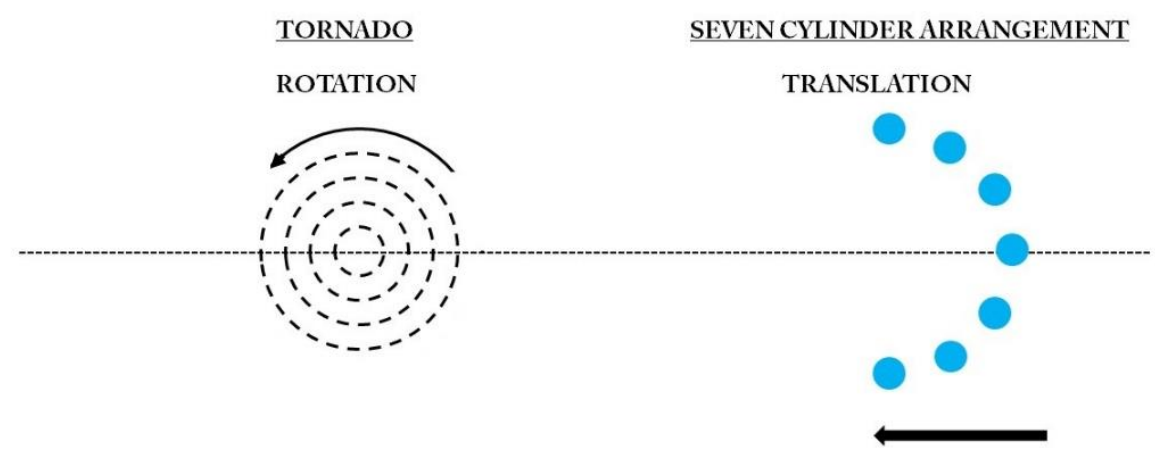

Figure 2.2 Customized RCVM

\subsection{Rotation Intensity of Tornadic Wind in RCVM}

An important parameter associated with the tornadic wind is the rotation intensity, denoted by $\beta$, which is the ratio of the maximum tangential velocity, $\left(V_{\gamma}\right)_{\max }$, to the translational velocity, $V_{t}$, of the tornado and is given by the following equation : 


$$
\left(V_{\gamma}\right)_{\max }=r_{c} \omega=\beta V_{t}
$$

The Eq. (2.5) clearly shows, the greater the value of $\beta$, greater is the dominance of the rotational component in the overall tornadic flow. 


\section{Chapter 3 - Numerical Methods}

\subsection{Lattice Boltzmann Method (LBM) With Multiple-Relaxation Time (MRT) Collision Technique}

Lattice Boltzmann Method (LBM) has emerged as a more successful and promising alternative to the conventional CFD approach of solving Navier-Stokes in the last two decades or so. LBM, having its root in the kinetic theory, is based on microscopic approach. Even the NavierStokes equations can be recovered from the Boltzmann equations by the use of collision operators such as single-relaxation time (SRT), multiple-relaxation time (MRT), and so on. This method consists of a large number of fictitious particles placed discretely at the nodes on a lattice mesh and the exchange of mass and momentum conservations is guided by the streaming and collision processes. In the streaming process, each fluid particle moves to the nearest node, whereas in the collision process, the particles coming to a node collide and change their velocity directions. LBM can be operated on different kind of lattices such as triangular, cubic, square, and so on.

In this study, the popular D2Q9[20] model is selected to operate on a square lattice and the collision is taken to be MRT technique. D2Q9 stands for two dimension, nine-velocity model. One of the important parameters in the LBM is the lattice speed, $c=\delta x / \delta t$, where $\delta x$ is the lattice spacing and $\delta t$ is the time step. Figure 3.1 represents the schematic of the D2Q9 square lattice model. Figure 3.1 illustrates that there is a total of 9 nodes, and a particle residing at a node can travel to any one of the nearest 8 neighbor nodes along any one of the eight connecting links for every time step. For every time step, the two processes, streaming and collision, would be implemented. 


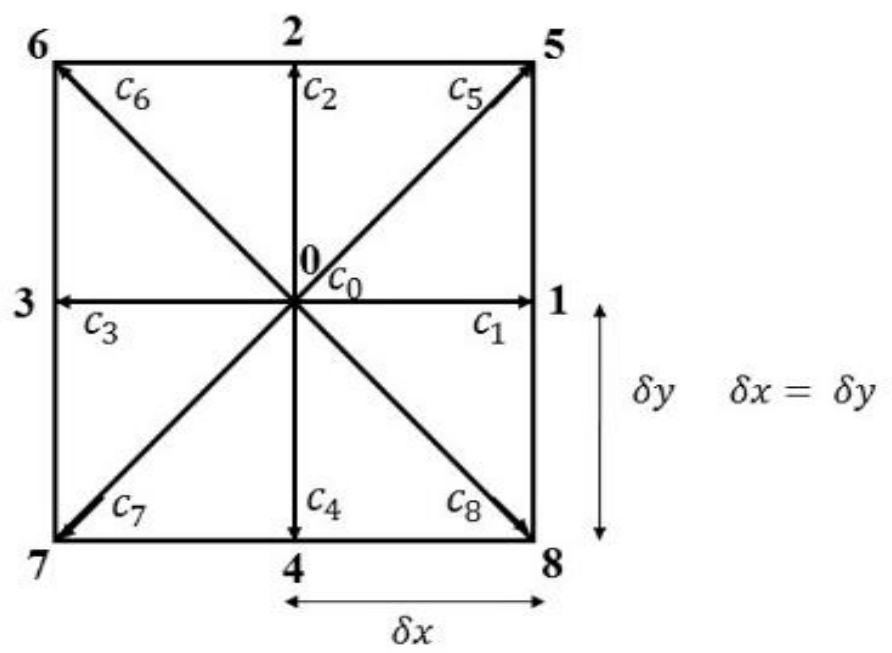

Figure 3.1 D2Q9 Square Lattice Model

The particle velocity set in the D2Q9 model is described by the particle distribution function[27] given below:

$$
c_{i}= \begin{cases}(0,0) & i=0 \\ (\cos [(i-1) \pi / 2], \sin [(i-1) \pi / 2]) c & i=1,2,3,4 \\ (\cos [(2 i-9) \pi / 4], \sin [(2 i-9) \pi / 4] \sqrt{2} c & i=5,6,7,8\end{cases}
$$

where $i$ represents the velocity direction in the lattice. Eq. (3.1) reveals that there are three types of particles in the D2Q9 model with the magnitudes of the velocity to be zero, $c$, and $\sqrt{2 c}$ respectively.

The governing equation of the MRT-LBM is given by Eq. (3.2):

$$
\boldsymbol{f}\left(x+c_{i} \delta_{t}, t+\delta_{t}\right)-\boldsymbol{f}(x, t)=-\boldsymbol{M}^{-1} \boldsymbol{S}\left[\boldsymbol{R}(x, t)-\boldsymbol{R}^{e q}(x, t)\right]
$$

where $\boldsymbol{f}$ is the distribution function vector, $\boldsymbol{R}$ is the moment vector, $\boldsymbol{R}^{e q}$ is the corresponding equilibrium moment vector, $\boldsymbol{M}$ is the transformation matrix, and $\boldsymbol{S}$ is the diagonal relaxation matrix. 
The relaxation matrix is given by Eq. (3.3):

$$
\boldsymbol{S}=\operatorname{diag}\left(s_{0}, s_{1}, \ldots \ldots \ldots \ldots, s_{6}, s_{7}, s_{8}\right)=\operatorname{diag}\left(0, s_{e}, s_{\varepsilon}, 0, s_{q}, 0, s_{q}, s_{v}, s_{v}\right)
$$

Here to reduce the cost of computation, $s_{0}=s_{3}=s_{5}=0$ is used. The values of the relaxation rates, $s_{e}, s_{v}$, and $s_{\varepsilon}$ can be adjusted within the interval $(0,2)$. The relation between the relaxation rates and the viscosities are given by Eq. (3.4) and (3.5):

$$
\begin{aligned}
& v=c_{s}{ }^{2}\left[\frac{1}{s_{v}}-\frac{1}{2}\right] \delta_{t} \\
& \zeta=c_{s}{ }^{2}\left[\frac{1}{s_{e}}-\frac{1}{2}\right] \delta_{t}
\end{aligned}
$$

where $v$ and $\zeta$ are the kinematic and bulk viscosities respectively, $c_{s}$ is the speed of sound and its value is taken to be one-third of the magnitude of velocity, c[27].

The distribution function vector is given by Eq. (3.6):

$$
\boldsymbol{f}=\left(f_{0}, f_{1}, f_{2}, f_{3}, f_{4}, f_{5}, f_{6}, f_{7}, f_{8}\right)^{\boldsymbol{T}}
$$

The moment and equilibrium moment vectors are given in the next two expressions as follows:

$$
\begin{gathered}
\boldsymbol{R}=\left(\rho, e, \varepsilon, j_{x}, q_{x}, j_{y}, q_{y}, p_{x x}, p_{x y}\right)^{\boldsymbol{T}} \\
\boldsymbol{R}^{e q}=\rho\left(1,-2+3 u^{2}, 1-3 u^{2}, u_{x},-u_{x}, u_{y},-u_{y}, u_{x}{ }^{2},-u_{y}{ }^{2}, u_{x} u_{y}\right)^{T}
\end{gathered}
$$

As the name suggests, the transformation matrix is used to transform the distribution function vectors in the forms given below:

$$
\begin{gathered}
\boldsymbol{R}=\boldsymbol{M} \boldsymbol{f} \\
\boldsymbol{R}^{e q}=\boldsymbol{M} \boldsymbol{f}^{e q}
\end{gathered}
$$


The transformation matrix itself is given by:

$$
\mathbf{M}=\left(\begin{array}{lllllllll}
1 & 1 & 1 & 1 & 1 & 1 & 1 & 1 & 1 \\
-4 & -1 & -1 & -1 & -1 & 2 & 2 & 2 & 2 \\
4 & -2 & -2 & -2 & -2 & 1 & 1 & 1 & 1 \\
0 & 1 & 0 & -1 & 0 & 1 & -1 & -1 & 1 \\
0 & -2 & 0 & 2 & 0 & 1 & -1 & -1 & 1 \\
0 & 0 & 1 & 0 & -1 & 1 & 1 & -1 & -1 \\
0 & 0 & -2 & 0 & 2 & 1 & 1 & -1 & -1 \\
0 & 1 & -1 & 1 & -1 & 0 & 0 & 0 & 0 \\
0 & 0 & 0 & 0 & 0 & 1 & -1 & 1 & -1
\end{array}\right)
$$

From Eq. (3.10), the corresponding equilibrium distribution function is found and is given by:

$$
f_{i}^{e q}=w_{i} \rho\left[1+\frac{\boldsymbol{c}_{i} \cdot \boldsymbol{u}}{c_{s}^{2}}+\frac{\left(\boldsymbol{c}_{i} \cdot \boldsymbol{u}\right)^{2}}{2 c_{s}^{4}}-\frac{u^{2}}{2 c_{s}^{2}}\right], i=0,1, \cdots, 8
$$

where

$$
w_{i}= \begin{cases}4 / 9 & i=0 \\ 1 / 9 & i=1,2,3,4 \\ 1 / 36 & i=5,6,7,8\end{cases}
$$

The density, velocity of the fluid, and the pressure can be found from the expressions below:

$$
\begin{gathered}
\rho=\sum_{i=0}^{8} f_{i} \\
\boldsymbol{u}=\frac{1}{\rho} \sum_{i=0}^{8} f_{i} c_{i} \\
p=\rho c_{s}^{2}
\end{gathered}
$$




\subsection{Integration of Immersed Boundary (IB) Scheme in LBM}

As already mentioned, while integrating the IB and LBM, the flow field $\boldsymbol{\Omega}$ is represented by a set of Eulerian points, which are fixed Cartesian points in the LBM framework. The boundary of the immersed object, denoted by $\boldsymbol{\Gamma}$, is represented by a set of Lagrangian points, $\boldsymbol{X}_{\boldsymbol{B}}^{\boldsymbol{l}}$, where $l$ and $B$ represent the Lagrangian point and the boundary of the immersed object respectively. An external force term reflecting the fluid-solid interaction, $f_{R S T}$, allocated at the Eulerian points within the neighborhood of the immersed boundary, should be numerically determined and then seamlessly added to the LBM framework. The IB and MRT-LBM incorporation is carried out through the following procedure:

Step 1: obtain the velocity $U_{B}^{l}$ at the Lagrangian points $\boldsymbol{X}_{B}^{l}$

To measure the closeness between an Eulerian point, $\boldsymbol{x}_{i j}=\left(x_{i}, y_{j}\right)^{t}$, and a Lagrangian point, $\boldsymbol{X}_{B}^{l}=\left(X_{B}^{l}, Y_{B}^{l}\right)^{t}$, the following continuous kernel distribution is required:

$$
D_{i j}\left(\boldsymbol{x}_{i j}-\boldsymbol{X}_{B}^{l}\right)=\delta\left(x_{i}-X_{B}^{l}\right) \delta\left(y_{j}-Y_{B}^{l}\right)
$$

where

$$
\delta(r)= \begin{cases}\frac{1}{4}\left(1+\cos \left(\frac{\pi|r|}{2}\right)\right), & |r| \leq 2 \\ 0, & |r|>2\end{cases}
$$

and the distribution is proposed by Peskin[37]. While $\boldsymbol{u}(\boldsymbol{x}, t)$ is known at all Eulerian points, the velocity of the fluid adhered to a Lagrangian point $\boldsymbol{X}_{B}^{l}$ can be determined by: 


$$
\boldsymbol{U}_{B}^{l}\left(\boldsymbol{X}_{B}^{l}, t\right)=\boldsymbol{u}\left(\boldsymbol{X}_{B}^{l}, t\right)=\sum_{i, j} \boldsymbol{u}(\boldsymbol{x}, t) D_{i j}\left(\boldsymbol{x}_{i j}-\boldsymbol{X}_{B}^{l}\right) \delta x \delta y
$$

with $\delta x=\delta y=1$ in the present LBM framework. Eq. (3.19) indicates that the fluid velocity at a boundary point is equal to the boundary velocity at the same position, which ensures no-slip condition at the boundary and no flow penetration.

Step 2: Determine the restoring force $\boldsymbol{F}_{R S T}^{l}$ at Lagrangian points $\boldsymbol{X}_{B}^{l}$

After $U_{B}^{l}$ is obtained at Lagrangian points, the restoring force per unit volume exerted at a Lagrangian point can be easily computed using the following feedback-forcing model[38]:

$$
\boldsymbol{F}_{R S T}^{l}=\alpha_{1} \int_{0}^{t}\left(\boldsymbol{U}_{B}^{l}-\boldsymbol{U}_{I M S}\right) d t+\alpha_{2}\left(\boldsymbol{U}_{B}^{l}-\boldsymbol{U}_{I M S}\right)
$$

where $\alpha_{1}$ and $\alpha_{2}$ are two negative free constants with dimensions of $M L^{-3} T^{-2}$ and $M L^{-3} T^{-1}$, respectively. According to [38], $\alpha_{1}$ and $\alpha_{2}$ are stable for moderate values within [-100,-1], and insensitive to their exact values. Also, note that $U_{I M S}$ in Eq. (3.20) represents the velocity of the immersed body, which can be defined by:

$$
\boldsymbol{U}_{I M S}(s, t)=\frac{\partial \boldsymbol{X}(s, t)}{\partial t}
$$

$U_{I M S}$ can be numerically determined using:

$$
\boldsymbol{U}_{I M S}(s, t)=\frac{\boldsymbol{X}(s, t)-\boldsymbol{X}(s, t-\delta t)}{\delta t}
$$

with $S$ denoting the Lagrangian parametric coordinate. Note that, for a stationary immersed body, $\boldsymbol{U}_{I M S} \equiv \mathbf{0}$ as $\boldsymbol{X}=\boldsymbol{X}(s)$ when the body does not move in the fluid. 
Step 3: Find the restoring force $f_{R S T}$ at Eulerian points $\boldsymbol{x}_{i j}$

After $\boldsymbol{F}_{R S T}^{l}$ is available at the Lagrangian points, the restoring force $f_{R S T}$ at Eulerian points, $\boldsymbol{x}_{i j}$ can be computed with the aid of continuous kernel distribution, as described in Eq. (3.17) and (3.18), as shown below:

$$
\boldsymbol{f}_{R S T}\left(\boldsymbol{x}_{i j}, t\right)=\sum_{l} \boldsymbol{F}_{R S T}^{l}\left(\boldsymbol{X}_{B}^{l}, t\right) D_{i j}\left(\boldsymbol{x}_{i j}-\boldsymbol{X}_{B}^{l}\right) \Delta s_{l}
$$

where $\Delta s_{l}$ is the arc length of the boundary segment.

Step 4: Embed the restoring force $f_{R S T}$ in LBM

In order to invoke $f_{R S T}$ in the LBM framework, the following transformation is employed:

$$
\tilde{f}_{i}=\left(1-\frac{1}{2 \tau}\right) w_{i}\left(\frac{c_{i}-u}{c_{S}^{2}}+\frac{\boldsymbol{c}_{\boldsymbol{i}} \cdot \boldsymbol{u}}{c_{S}^{4}}\right) \cdot \boldsymbol{f}_{R S T}
$$

where $w_{i}$ represents the coefficients in the equilibrium distribution function mentioned in Eq. (3.13).

The inclusion of $\widetilde{f}_{l}$ leads to the following revised LBE[39]:

$$
f_{i}\left(x+c_{i} \delta t, t+\delta t\right)-f_{i}(x, t)=-\frac{1}{\tau}\left[f_{i}(x, t)-f_{i}^{e q}(x, t)\right]+\widetilde{f}_{l} \delta t
$$

Finally, the velocity in the LBM framework has to be updated by including $f_{R S T}$ as follows:

$$
\rho \boldsymbol{u}=\sum_{i=0}^{8} f_{i} \boldsymbol{c}_{i}+\frac{1}{2} \boldsymbol{f}_{R S T} \delta t
$$




\section{Chapter 4 - Important Parameters, Numerical Experiments, and}

\section{Computational Domain Setup}

\subsection{Definitions of Important Parameters}

\subsubsection{Orientation Angle}

One of the major subjects of this research is to investigate the wind-loadings on the 7-cyinder model at different orientation angles. Before the investigation, the orientation angle has to be defined first. Figure 4.1 shows the arrangement of seven cylinders and the definition of the orientation angle, $\theta$. The orientation angle is defined as the angle between the line of symmetry of the 7-cylinder arrangement passing through the center of middle cylinder and the initial translational direction of the path of tornado and is measured counter-clockwise. Due to concurrent translation and rotation, the translation direction of the tornado keeps varying. As the tornado rotates anticlockwise and translates to the right as shown in the Figure 4.1, it would be moving upwards and to the right. Therefore, the translation direction of the tornado during its initial setup in the computational domain has been taken as the reference for the calculation of orientation angle.

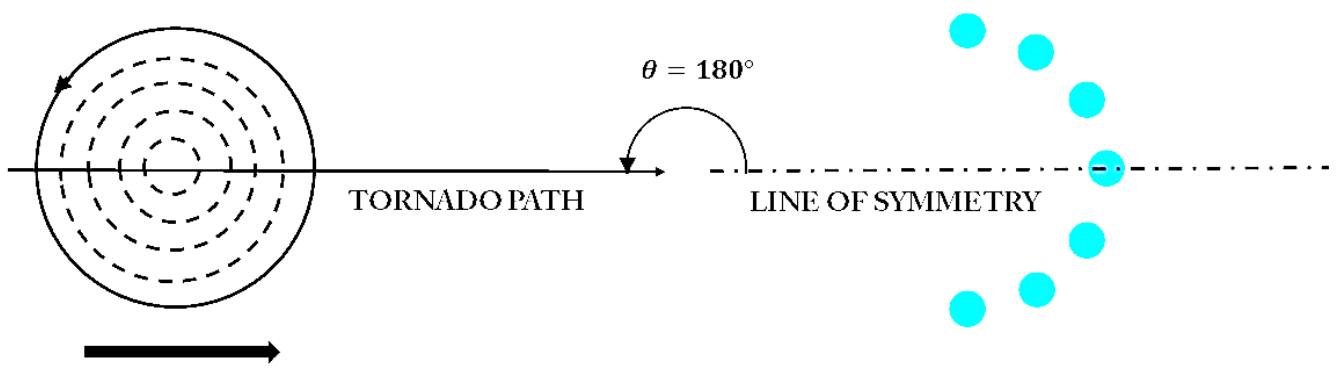

Figure 4.1 Illustration of orientation angle 


\subsubsection{Force Coefficient in $\mathrm{X}$ - direction}

Force coefficient in $\mathrm{X}$-direction, $C_{x}$, is defined as the ratio of the summation of forces acting on the body in the X-direction, $F_{x}$, to the product of the dynamic pressure and the reference length of the body. Here, the diameter of the cylinders, D, used in the model is taken as the reference length. Eq. (4.1) shows the mathematical definition of $C_{x}$ as given below:

$$
C_{x}=\frac{F_{x}}{0.5 \rho V_{t}^{2} D}
$$

\subsubsection{Force Coefficient in $\mathrm{Y}$ - direction}

Eq. (4.2) shows the definition of the force coefficient in Y-direction, $C_{y}$, as given below:

$$
C_{y}=\frac{F_{y}}{0.5 \rho V_{t}^{2} D}
$$

where $F_{y}$ is the summation of forces on the body in the Y-direction.

\subsubsection{Moment Coefficient}

The definition of moment coefficient, $C_{m}$, is given by the Eq. (4.3) and it differs from the definition of force coefficients in such a way that the reference area of the body has replaced the reference length of the body. Here, the reference area is taken as the cross-sectional area of the cylinders used in the model. The resultant moment, $\mathrm{M}$, acting on the body is termed positive when it is counter-clockwise and vice-versa. The resultant moment on the 7 cylinders are calculated about their own centers.

$$
C_{m}=\frac{M}{0.5 \rho V_{t}^{2}\left(\pi D^{2} / 4\right)}
$$




\subsubsection{Resultant Coplanar Force Coefficient}

Another important parameter that needs be defined and discussed which concerns the total force acting on the buildings is the resultant coplanar force coefficient, $C_{F}$, and is given by the following Eq. (4.4):

$$
C_{F}=\sqrt{\frac{F_{x}^{2}+F_{y}^{2}}{\left(0.5 \rho V_{t}^{2} D\right)^{2}}}
$$

The higher the value of $C_{F}$ in Eq. (4.4), the greater is the value of resultant force acting on the building. The greater resultant force is an indication of the severity of the tornado on the building and it necessitates the buildings to be constructed with a proper design which accommodates both the rotational and translational components of the tornado.

\subsection{Numerical Experiments}

As already mentioned, the building model used in the numerical experiments of this research is a 7-cylinder model with the seven cylinders arranged in a semi-circle and are separated by an angle of 30 degrees. The cylinders are numbered clockwise from 1 to 7 with line of symmetry

passing through the center of cylinder 4 . The numerical experiments include seven test cases with the model placed at orientation angles from $0^{\circ}$ to $180^{\circ}$. Figure 4.2 shows the schematic of the test cases A to $\mathrm{G}$ and the initial direction of the path of the tornado along with the value of orientation angles for each case. It is obvious that the initial translation direction of the tornado is parallel to one cylinder in every test case. In the series of numerical experiments, all the four wind-loading coefficients have been recorded for all the seven test cases A to G. 


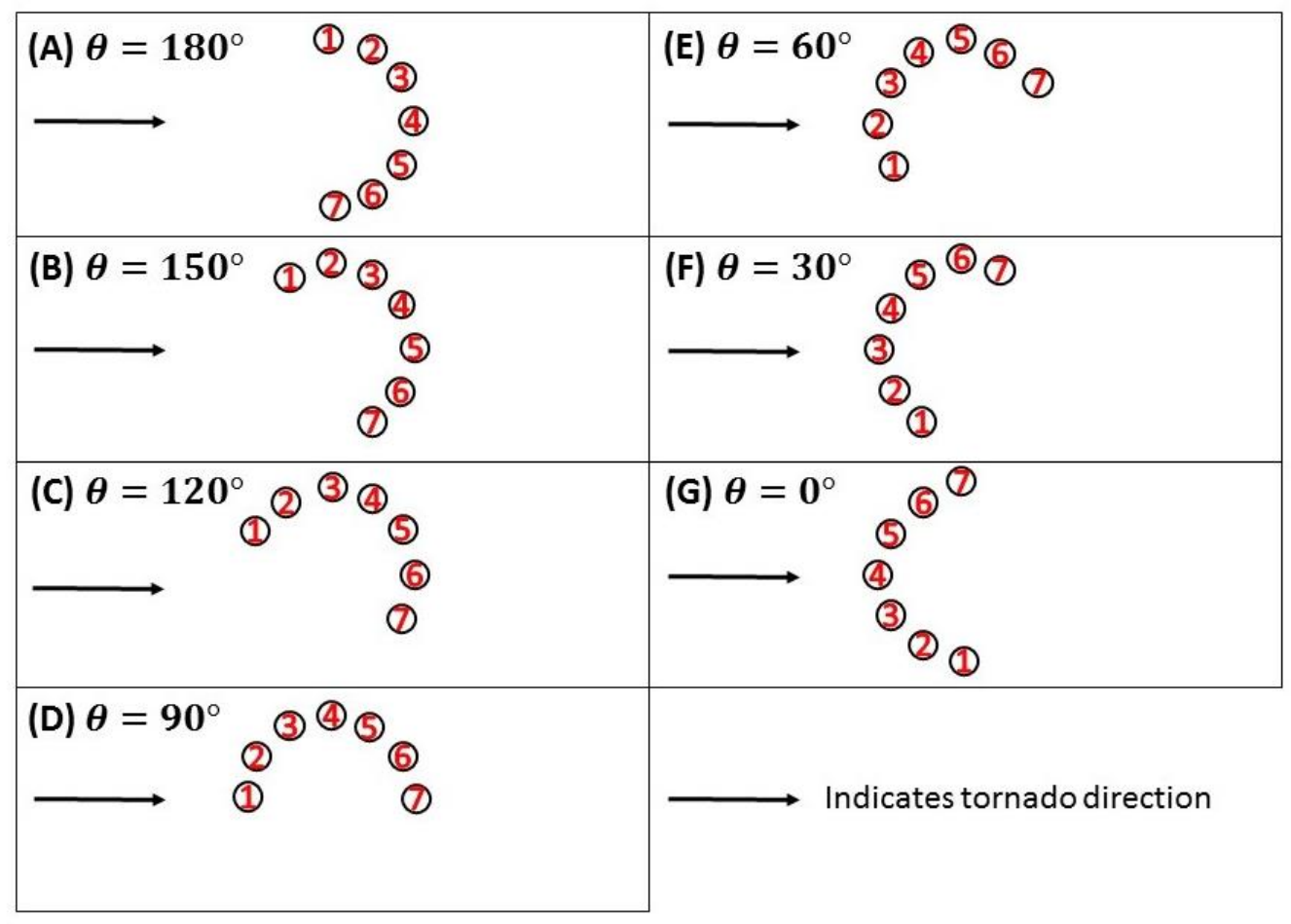

Figure 4.2 Illustration of 7 test cases $A$ to $G$ and their orientation angles

\subsection{Computational Domain Setup}

Figure 4.3 illustrates the setup of the computational domain. It is important to realize that five mesh resolutions at $1 / 40,1 / 50,1 / 60,1 / 80$, and $1 / 100$ were tested to confirm that the $1 / 50$ grid resolution remains a suitable choice, reiterating the conclusion the conducted grid independence study[40]. The LBM framework is operated on a $[-40,40] \times[-15,15]$ square lattice. The flow field parameters Reynolds number, defined based on the translational velocity of the tornado and the diameter of the cylinder, and density have been assigned the values of 1000, and 1 unit respectively. The radius of the core of the tornado, $r_{c}$, is chosen as 150 units with the center fixed at $(0,0)$. The angular velocity, $\omega$, of the tornado is taken as $0.00068 \mathrm{rad} / \mathrm{s}$ in the anticlockwise direction in order to obtain the rotation intensity of 3.0. Using the immersed boundary approach, the seven cylindrical buildings are constructed with a diameter, D, of 50 units each and there are 
200 Lagrangian points taken around each building, which is given a translational velocity, $V_{t}$, of 0.034 units/s towards the tornado. As per the numerical procedure, the translational velocity of the building is equal in magnitude and opposite in direction to the translational velocity of the tornado. The seven buildings are arranged in a semi-circle with the center initially placed at $(34,0)$ and a diameter of '3D' units, which is equal to the diameter of the tornado.

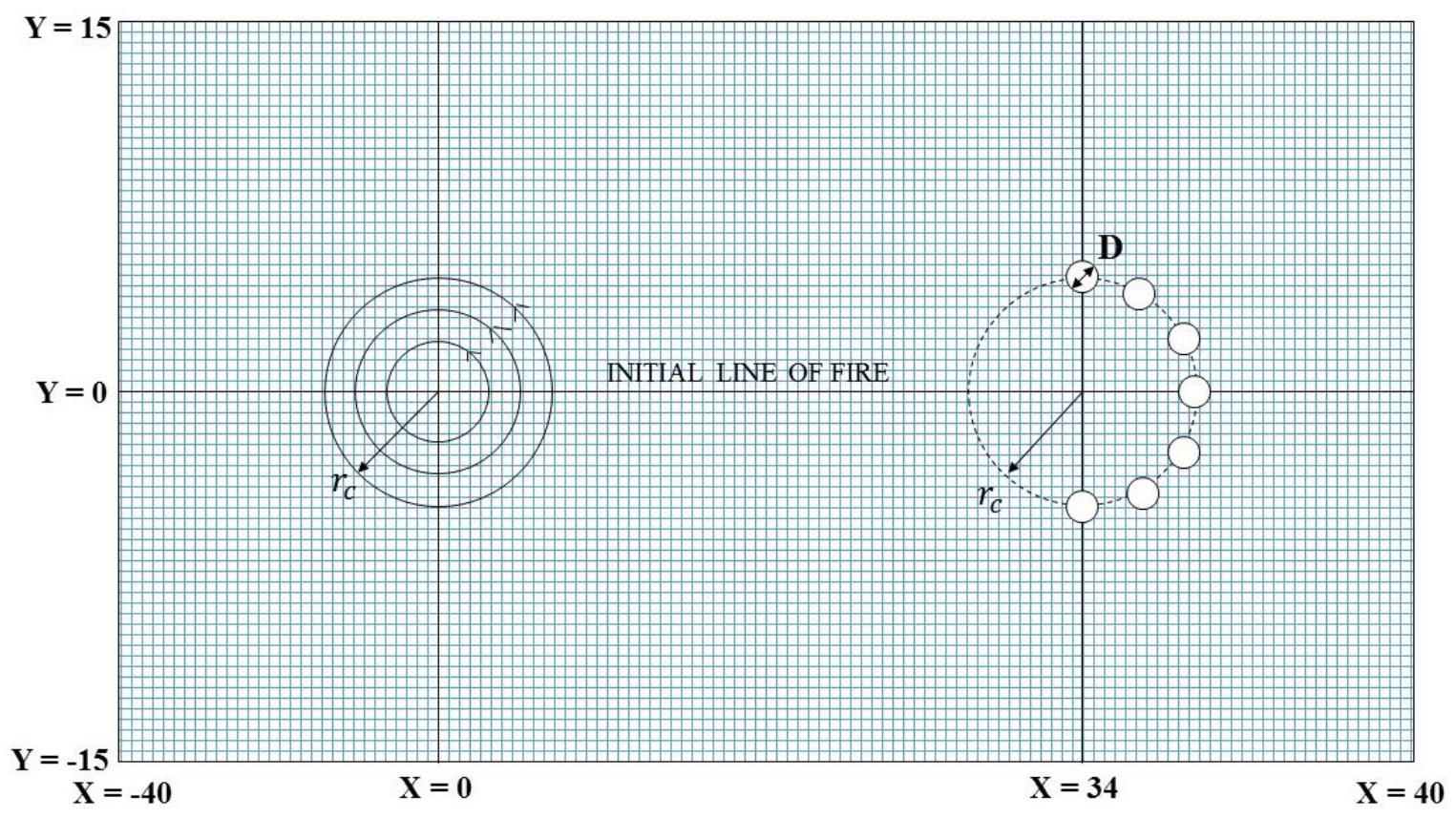

Figure 4.3 Setup of the computational domain 


\section{Chapter 5 - Test A Case Study}

\subsection{Three Stages of Tornado - Structure Interaction}

Before explaining the numerical results, three important stages of the tornado-building interaction have to be defined, which is the key to the understanding of the interaction. The three stages are as follows: 1) Pre-Interaction stage, 2) Primary interaction stage, and 3) Post-Interaction stage. Pre-Interaction stage, as the name suggests, represents the time period before the buildings enter into the core of the tornado. The primary interaction stage represents the time period in which the building is encircled by the core of the tornado. In this stage, the building is expected to experience the higher wind-loadings that makes this stage to be analyzed more in detail. It is important to remember that the actual interaction between the tornado and the buildings commences well before the primary interaction stage, i.e., in the pre-interaction stage itself. Finally, the post-interaction stage starts once the building comes out of the core of the tornado. In this stage, the effect of tornado on the building subsides and its deteriorating action is likely to be abated.

Figure 5.1, Figure 5.2, and Figure 5.3 show the variation of the aerodynamic coefficients, $C_{x}$, $\mathrm{C}_{\mathrm{y}}$ and $\mathrm{C}_{\mathrm{m}}$, with $\mathrm{X}$, respectively, for the test case, where ' $\mathrm{X}$ ' indicates the distance between the center of the tornado and the center of the cylindrical building; $\mathrm{X}=0$ indicates the time instant at which the centers of the tornado and the virtual circle on which the 7 cylinders are placed coincide when the translational velocity of the tornado alone is taken into account; in the intervals $\mathrm{X}<0$ and $\mathrm{X}>0$, the tornado is approaching and leaving the building, respectively. 


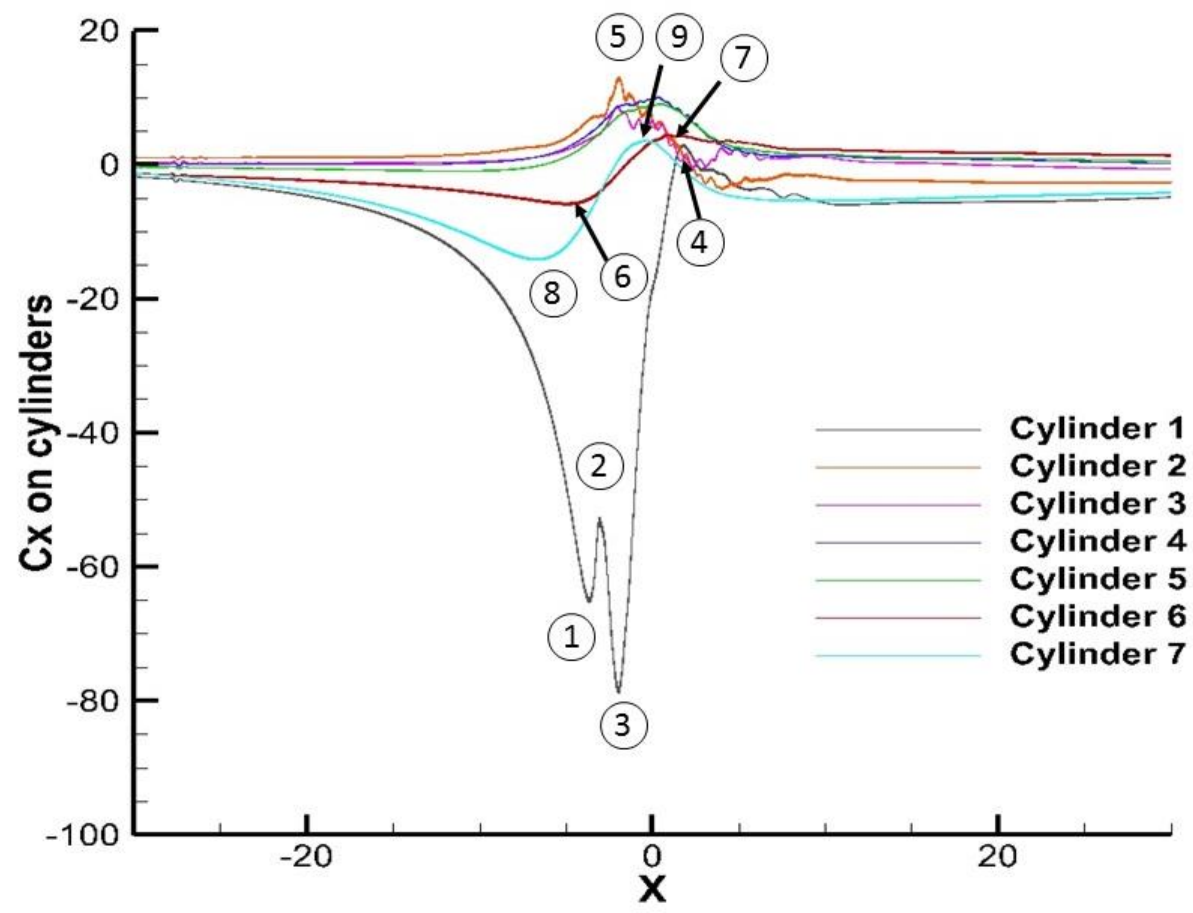

Figure 5.1 Variation of $C_{x}$ with $X$ for case $A$

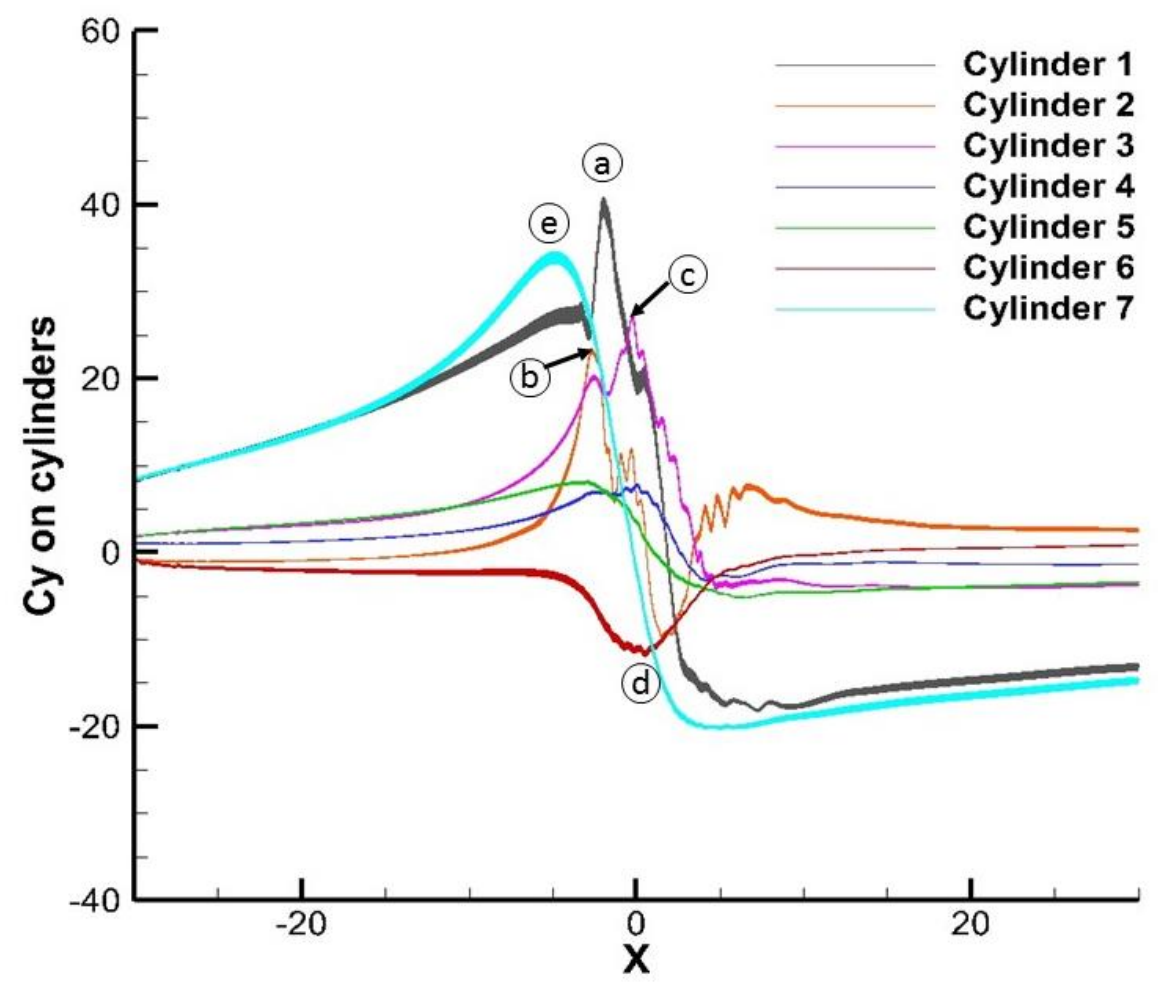

Figure 5.2 Variation of $C_{y}$ with $X$ for case $A$ 


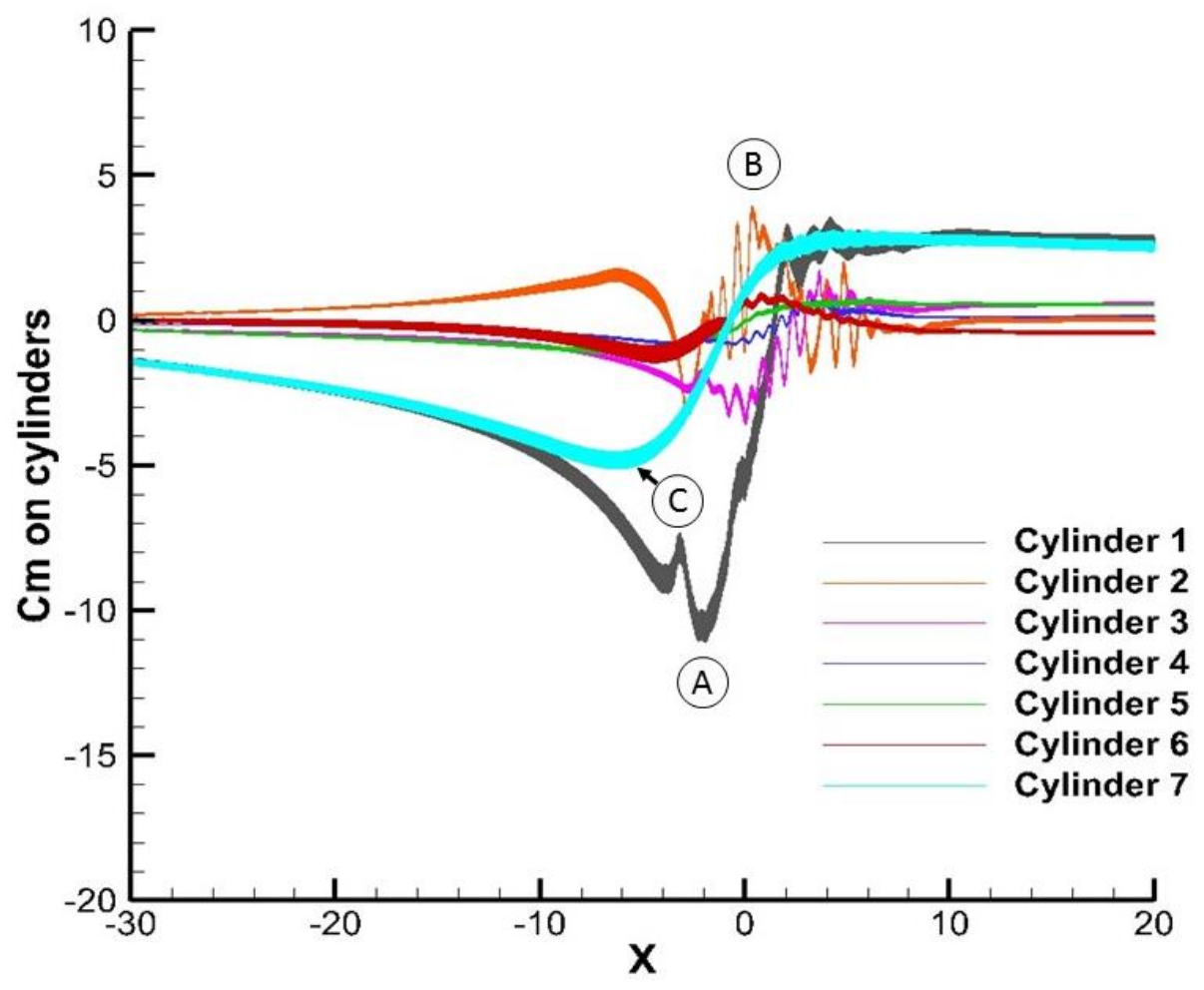

Figure 5.3 Variation of $C_{m}$ with $X$ for case $A$

\subsection{Analysis of $C_{x}$ Curves}

In Figure 5.1, $\mathrm{C}_{\mathrm{x}}$ vs $\mathrm{X}$ curve, it is observed that the cylinder 1 experiences the highest magnitude of $\mathrm{C}_{\mathrm{x}}$ compared to other cylinders and there are four major turning points on cylinder 1 curve marked as points (1) to (4). In the pre-interaction stage $(X<-3)$, the $C_{x}$ curve of cylinder 1 decreases very gradually in the initial stages $(X<-10)$ and then decreases abruptly, as the cylinder 1 gets closer to the tornado in the final stages $(-10<X<-3)$ and reaches the local minimum at point (1), just before $X=-3$. This difference in slopes in the pre-interaction stage can be explained according to the RCVM Eq. (2.1) and (2.5), $V_{\gamma}=\frac{r_{c}^{2} \omega}{r}=\beta V_{t} \frac{r_{c}}{r}$, where $r_{c}, \beta$, and $V_{t}$ are constants and, $V_{\gamma}$ is inversely proportional to $r$. In the pre- interaction stage, $r>r_{c}$; when $\mathrm{X}=-3, r$ becomes equal to $r_{c}$, approximately. As the cylinder 1 moves towards the tornado, $r$ 
value decreases and $V_{\gamma}$ increases. Thus, the increase in upward tangential velocity of the tornado increases the magnitude of $\mathrm{C}_{\mathrm{x}}$ abruptly that produces the steep slope in the final phase of the preinteraction stage. Negative value of $\mathrm{C}_{\mathrm{x}}$ indicates that the cylinder is pulled towards the center of the tornado in the negative $\mathrm{X}$-direction. After reaching the local minimum at point (1), the $\mathrm{C}_{\mathrm{x}}$ curve increases and achieves a local maximum at point (2), after which $\mathrm{C}_{\mathrm{x}}$ drops again to attain a global minimum at point (3), where $X=-2$; then, it re-rises monotonically to achieve a local maximum value at point (4), just a bit before $X=2$. After the point (4), the curve decreases for a shorter while and then becomes almost steady. The four turning points, (1) to (4), are examined on the basis of flow visualization using velocity magnitude contours represented in the Figure 5.4, Figure 5.5, Figure 5.6, and Figure 5.7 respectively.

In Figure 5.4, Figure 5.5, and Figure 5.6, it can be easily seen that the cylinder 1 enters the right upper core region of the tornado. The left half of the cylinder 1 encounters a higher tangential velocity than the right half as per the Eq. (2.1) in the RCVM and therefore the cylinder 1 experiences a negative $C_{x}$ at the three points (1), (2), and (3). But the values of $C_{x}$ at these three points are not same and that can be explained on the basis of the relative positions of cylinder 1 with the tornado and the interference effects of other cylinders on cylinder 1. A careful comparison of the Figure 5.4 and Figure 5.5 unveils that the cylinder 1 at point (2) is closer to the tornado center than at point (1). It is expected that the magnitude of $C_{x}$ increases further from point (1) to (2), but the scenario is exactly opposite. The decrease in magnitude of $C_{x}$ at point (2) is attributed to the interference effects of essentially the cylinders 2 and 7 on cylinder1, which can be corroborated from Guo[41], in which the $C_{x}$ curve does not show any bump in the primary interaction stage for a cylinder in a tornado. The more tornado core flow rate deflected off the cylinders 2 and 7 has passed around cylinder 1 and created a vaguely-defined, wide-spread loop 
of relatively lower velocity region in front of cylinder 1 in Figure 5.5 when compared to a welldefined loop of relatively higher velocity region in Figure 5.4. This difference in the high velocity loop region is responsible for the higher $\mathrm{C}_{\mathrm{x}}$ magnitude associated with cylinder 1 at point (1). An observation of the Figure 5.6 tells that the cylinder 1 is very closer to the center of the tornado and there is a clear cut existence of high velocity and low velocity wake regions in the front and rear of the cylinder 1 , which leads to the global minimum $C_{x}$ at point (3). The magnitude of the velocity in the wake region behind the cylinder 1 is slightly lower in the case of point (3), when compared to point (1), and this little velocity difference gives a higher pull on cylinder 1 at point (3) and makes it the global minimum point. Finally, in Figure 5.7, corresponding to point (4), the cylinder 1 lies in the lower left core portion of the tornado. The left portion of the cylinder 1 is exposed to a lower tangential velocity than the right portion as per Eq. (2.1) and this is clearly evident in Figure 5.7 and thereby, cylinder 1 experiences a push force in the positive X-direction at point (4). But the distorted velocity pattern of the tornado core shows its inability to produce higher loading, but a very low positive value of $\mathrm{C}_{\mathrm{x}}$.

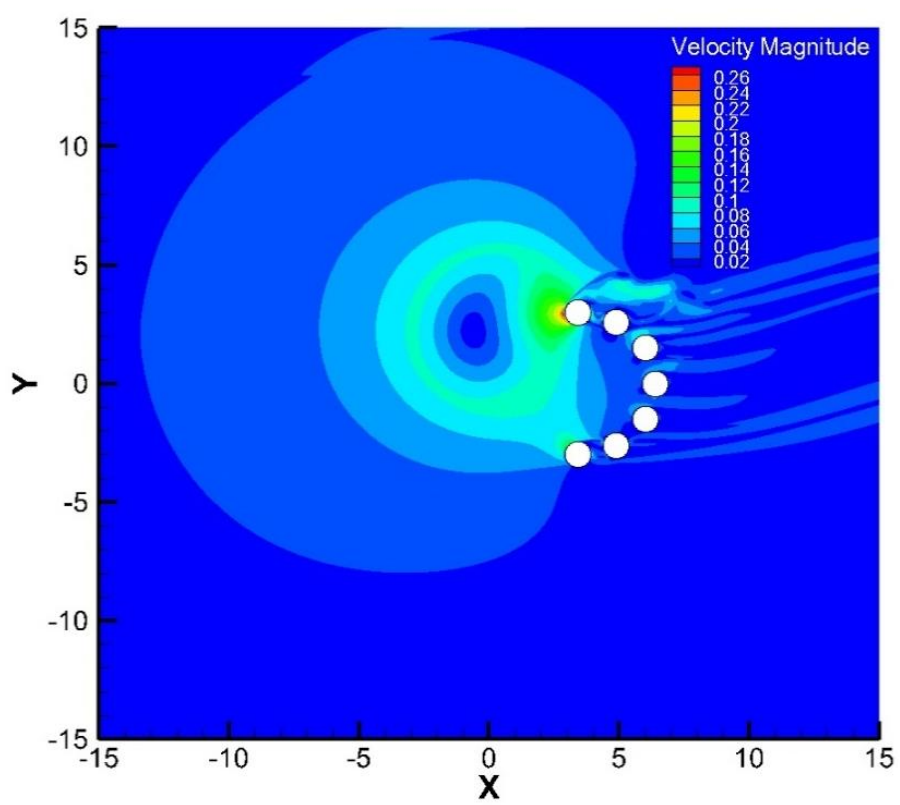

Figure 5.4 Velocity magnitude contour corresponding to point (1) of Figure 5.1 (Pg. No. 27) 


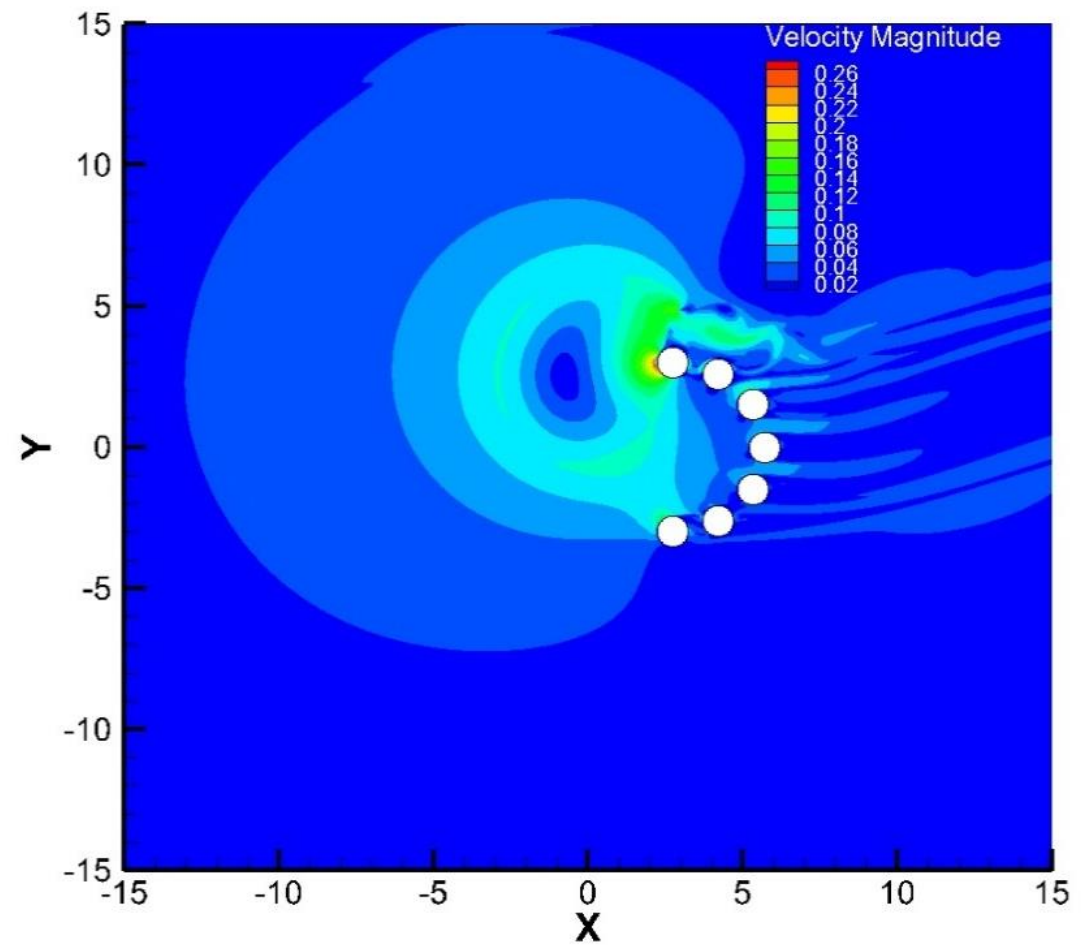

Figure 5.5 Velocity magnitude contour corresponding to points 1) (2) of Figure 5.1 (Pg. No. 27) 2) (b) of Figure 5.2 (Pg. No. 27)

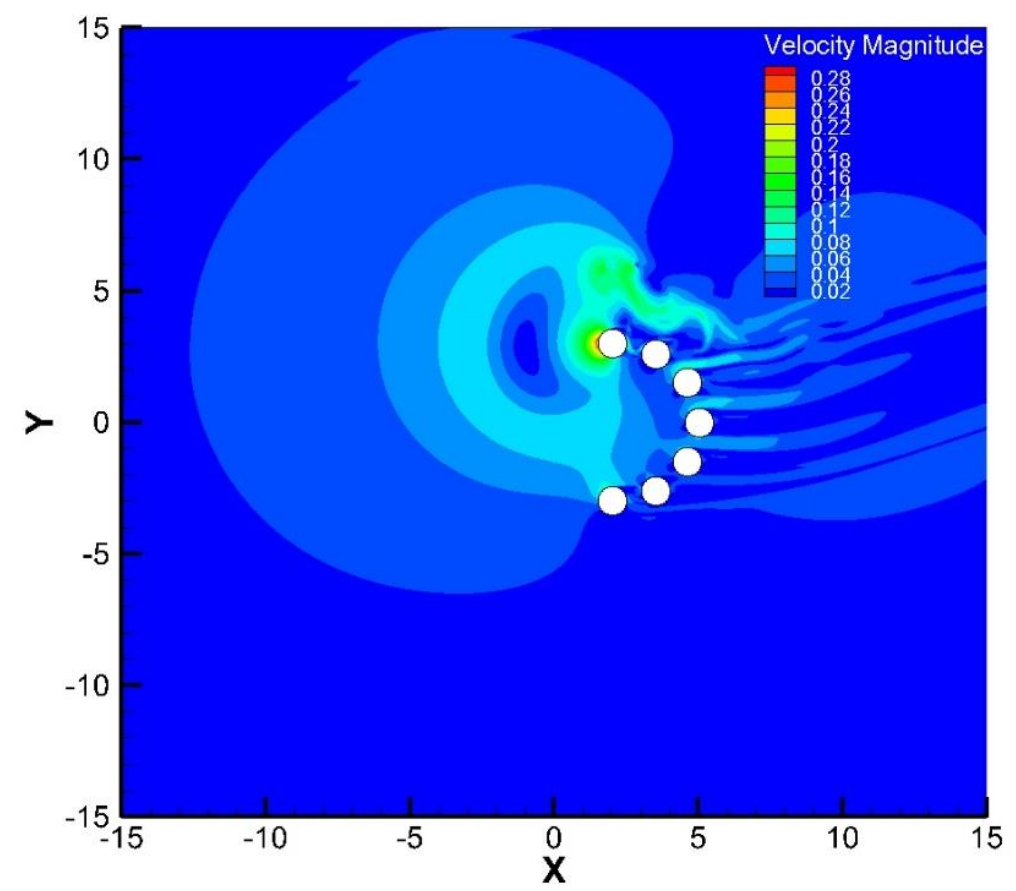

Figure 5.6 Velocity magnitude contour corresponding to points 1) (3) of Figure 5.1(Pg. No. 27) 2) (5) of Figure 5.1(Pg. No. 27) 3) (a) of Figure $5.2(P g . N o .27) 4)$ (A) of Figure 5.3 (Pg. No. 28) 


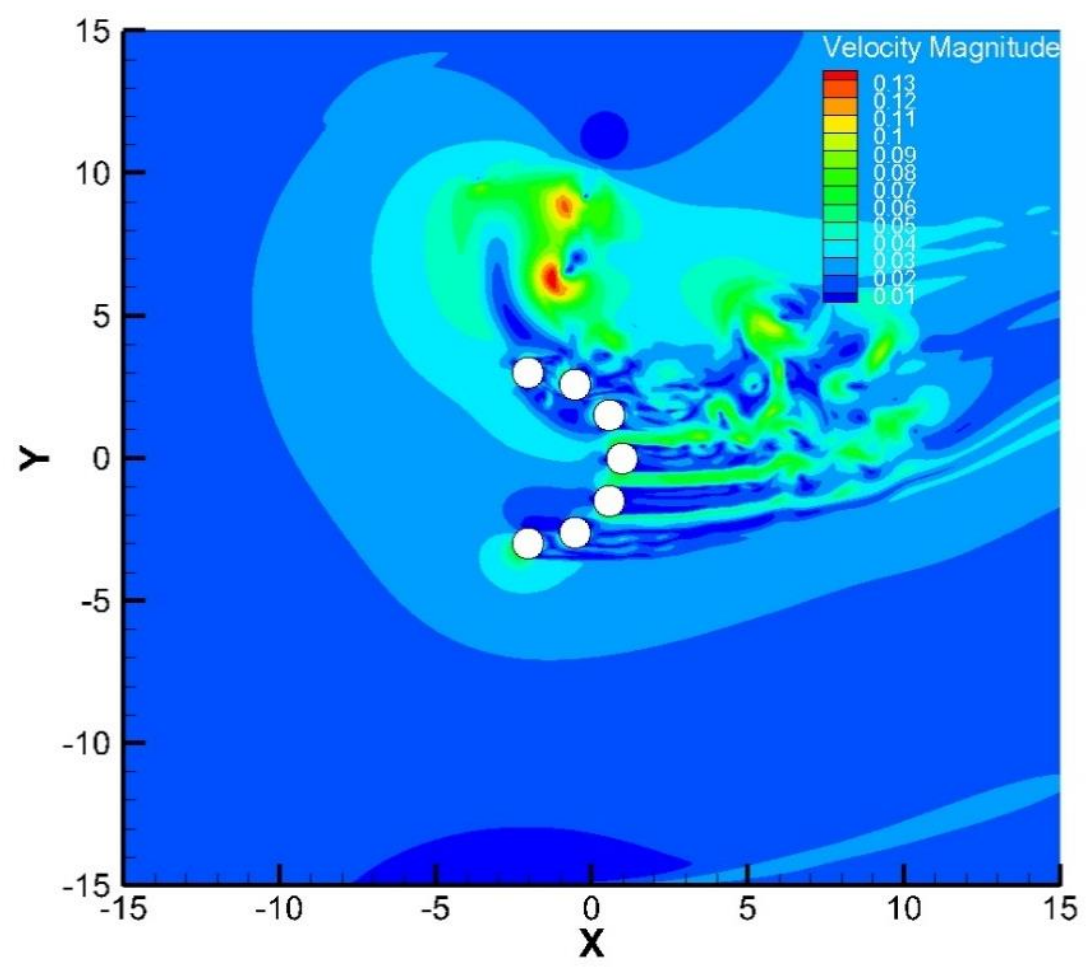

Figure 5.7 Velocity magnitude contour corresponding to point (4) of Figure 5.1 (Pg. No. 27)

After investigating the $C_{x}$ characteristics of cylinder 1, the focus is now moved on to the $C_{x}$ characteristic of cylinder 2. In Figure 5.1, the $\mathrm{C}_{\mathrm{x}}$ curve of cylinder 2 does not show any trace of the characteristics shown by cylinder 1 . The $C_{x}$ curve increases very slowly and achieves global maximum at point (5) and then decreases to a local minimum, after which it attains a steady value. An observation of the cylinder 2 curve divulges a lot of oscillations on it and these oscillations are attributed to the interference effects of the other cylinders on cylinder 2, especially cylinder 1 . Moreover, these oscillations are achieved by the incorporation of Large Eddy Simulation (LES)[42] turbulence model in the Lattice Boltzmann Method. Recalling Figure 5.6, corresponding to point (5), shows the cylinder 2 lying in the upper right core portion of the tornado. Cylinder 2 is anticipated to experience higher pull force as per the Eq. (2.1), but it experiences a push force of smaller magnitude at point (5). A careful observation of Figure 5.6 clearly shows 
some right portion of the core of tornado completely distorted and destroyed by the interference of cylinders 1 and 7 and moreover, the cylinder 2 lies in the wake of the cylinder 1, which created the push force.

A similar examination of the $C_{x}$ curves of the cylinders 3 to 5 also reveals a plethora of oscillations on those curves, which is a clear indication of the interference phenomenon. An examination of the Figure 5.4 and Figure 5.7 discloses the fact there are no appreciable velocity differences created on the front and rear of the cylinders 3 to 5 . This is due to the fact that the core of the tornado has been completely distorted by the other cylinders before the cylinders 3 to 5 entered into the core.

After analyzing the cylinders 3 to 5 , the attention is now on the $\mathrm{C}_{\mathrm{x}}$ characteristics of the cylinders 6 and 7. In Figure 5.1, the $C_{x}$ curves of cylinder 6 and 7 follows a similar trend of decreasing gradually in the pre-interaction stage; after which they reach local minimum at points (6) and (8); then, they increase and attain local maximum at points (7) and (9); but, the cylinder 6 experiences lesser $C_{x}$ values than the cylinder 7. Figure 5.8 and Figure 5.9 represent the velocity magnitude contours at the points (6) and (7) of Figure 5.1, respectively. A careful examination of the Figure 5.8 shows the cylinder 6 lying in the lower right portion of the core of tornado and experiences a pull force as expected at point (6). But the very low $C_{x}$ value is due to the fact that the cylinder 6 has not entered the core of the tornado and the interference of cylinder 7 that deflects the core flow away from the cylinder 6 which is evident in Figure 5.8. In Figure 5.9, corresponding to point (7), the cylinder 6 lying away from the core of the tornado experiences a very small push force in the positive X-direction. In Figure 5.10, the cylinder 7 occupies the lower right portion of the core. As per Eq. (2.1), the cylinder 7 experiences higher pull force towards the core of the tornado at point 
(8). In Figure 5.11, corresponding to point (9), the cylinder 7 is approximately lying at the edge of the core and right below the center of tornado and very low positive $C_{x}$ is experienced.

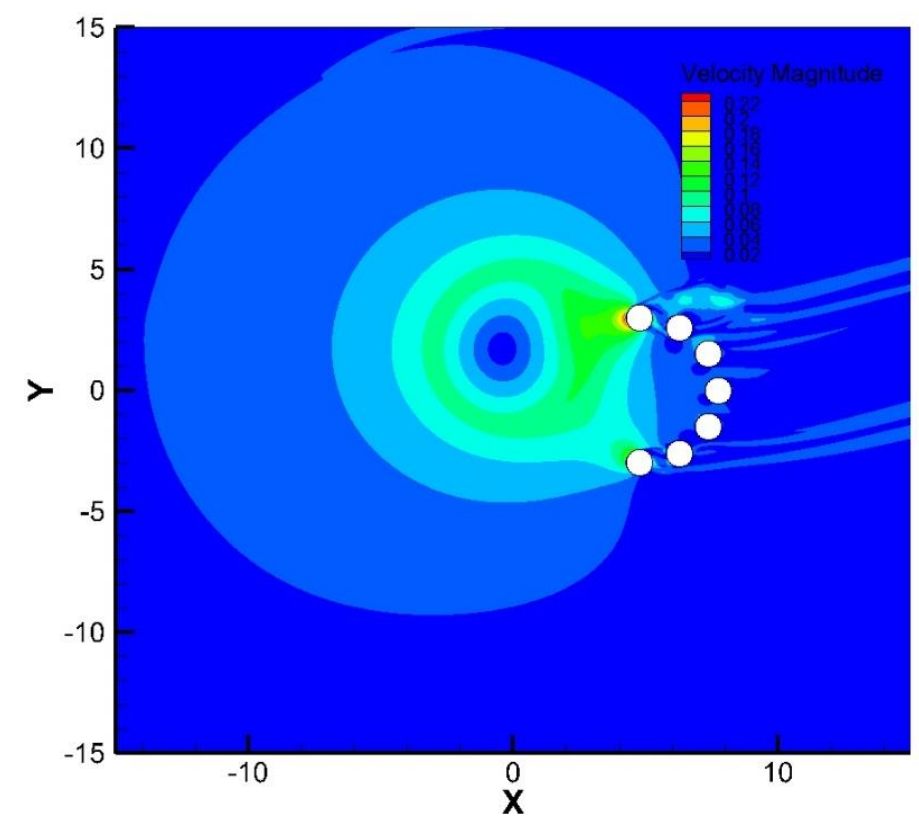

Figure 5.8 Velocity magnitude contour corresponding to points 1) (6) of Figure 5.1 (Pg. No. 27) 2) (e) of Figure 5.2 (Pg. No. 27)

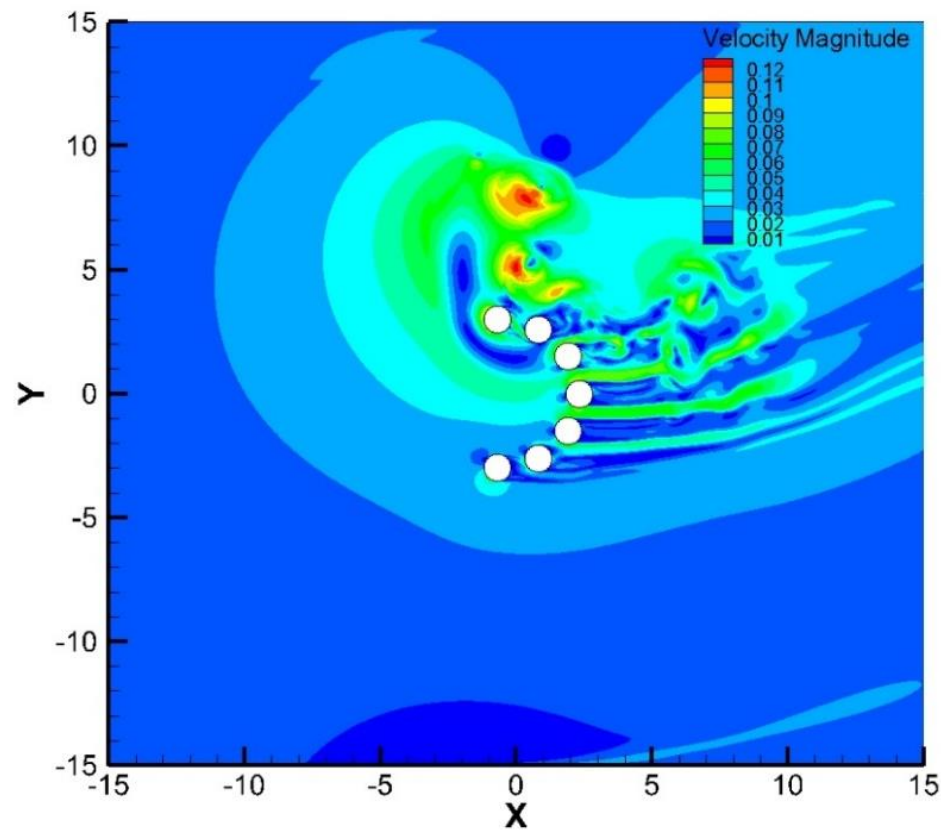

Figure 5.9 Velocity magnitude contour corresponding to points 1) (7) of Figure 5.1 (Pg. No. 27) 2)

(d) of Figure 5.2 (Pg. No. 27) 3) (B) of Figure 5.3 (Pg. No. 28) 


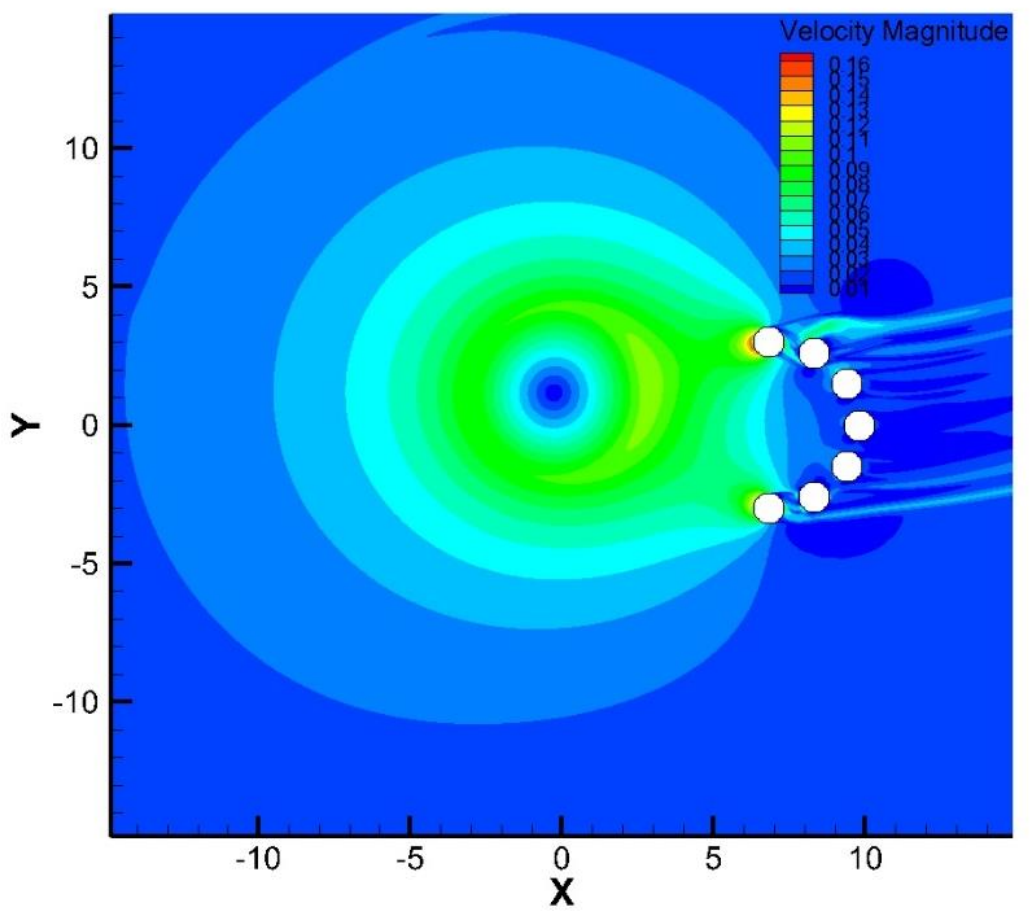

Figure 5.10 Velocity magnitude contour corresponding to points 1) 8) of Figure 5.1 (Pg. No. 27) 2) (C) of Figure 5.3 (Pg. No. 28)

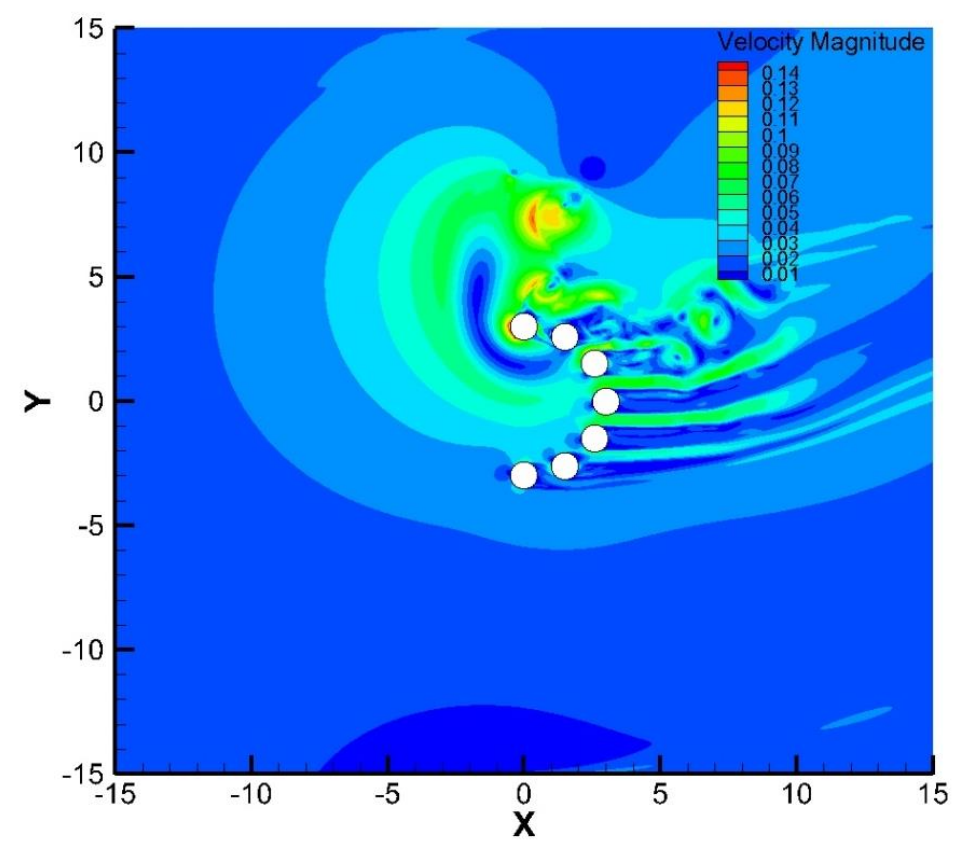

Figure 5.11 Velocity magnitude contour corresponding to points 1) (9) of Figure 5.1 (Pg. No. 27) 2) (C) of Figure 5.2 (Pg. No. 27) 


\subsection{Analysis of $C_{y}$ Curves}

After explaining the salient points in the $C_{x}$ vs $X$ graph, the focus is now on the $C_{y}$ vs $X$ curve depicted in Figure 5.2. An observation of the curves in Figure 5.2 just reveals that the overall trend of the $\mathrm{C}_{\mathrm{y}}$ curves is in complete contrast with the trend observed by the $\mathrm{C}_{\mathrm{x}}$ curves. The $\mathrm{C}_{\mathrm{y}}$ curves, in general, increases gradually in the pre-interaction stage and then rises steeply to achieve local maximum in the primary interaction stage, after which the curve falls and finally flattens out in the post-interaction stage. A careful study of the curves tells that the cylinder 1 again has suffered the maximum followed by cylinder 7 with the second highest maximum. As already seen with $C_{x}$ curves of cylinders 3 to 5 , the $\mathrm{C}_{\mathrm{y}}$ curves of cylinders 3 to 5 also have lot of fluctuations, but the fluctuations are more severe in the case of $\mathrm{C}_{\mathrm{y}}$ curves that corroborates the interference phenomenon explained already in the previous section.

An observation of Figure 5.6, corresponding to point (a) in Figure 5.2, shows the cylinder 1 in the upper right portion of the core of the tornado. Due to the anti-clockwise rotation of the tornado, the cylinder 1 experiences upward tangential velocity on its lower portion that produces high pressure region at its bottom which in turn creates an uplift in the positive Y-direction at point. It is noted that the global minimum $\mathrm{C}_{\mathrm{x}}$ and global maximum $\mathrm{C}_{\mathrm{y}}$ values experienced by cylinder 1 at points (3) and (a) respectively happen at the same time step.

After looking at the cylinder 1 characteristics, the attention is now on the $\mathrm{C}_{\mathrm{y}}$ characteristics of cylinder 2. An observation of the cylinder 2 curve in Figure 5.2 unveils a local maximum point, marked as (b). An examination of the Figure 5.5, corresponding to point (b), clearly shows the upper portion of the cylinder 2 lying in the wake region of the cylinder 1 . This wake region creates a low pressure region on top of cylinder 2 that creates an uplift on cylinder 2 at point (b). 
An inspection of the cylinder 3 curve reveals a local maximum at point (c) of Figure 5.2. Figure 5.11, corresponding to point (C), apparently shows a low pressure region on top of cylinder 3 in the form of a vortex created by cylinder 2 and this accounts for an uplift in the Y-direction. After cylinder 3, the analysis is made on the cylinder 6 curve in which the fall characteristic is more pronounced than the rise characteristic and a salient point is marked as point (d), a local minimum point. After scrutinizing the Figure 5.9, corresponding to point @), it is noted that the cylinder 6 lies outside the core of the tornado and in the region below the tornado. As per Eq. (2.1), the bottom portion of the cylinder 6 is exposed to higher tangential velocity than the top portion, thus creating a down lift on cylinder 6 at point (d).

A careful observation of the cylinder 7 curve in Figure 5.2 reveals a local maximum point at point (e). Figure 5.8, corresponding to point $(e$, shows cylinder 7 lying in the lower right portion of the core of the tornado. As per Eq. (2.1), the top portion of the cylinder 7 is exposed to the higher tangential velocity than the bottom portion and this creates an uplift on cylinder 7 at point (e)

\subsection{Analysis of $C_{m}$ Curves}

The $C_{m}$ curves, in general, follows the same trend as the $C_{x}$ curves. The $C_{m}$ curve decreases gradually in the pre-interaction stage and then decreases abruptly in the primary interaction stage; after which it increases to achieve maximum and then decreases again for a while before it becomes steady.

The $\mathrm{C}_{\mathrm{m}}$ curve of cylinder 1 shows a global minimum point marked as point (A) in Figure 5.6. It shows the cylinder 1 lying in the upper right portion of the core of the tornado. As per Eq. (2.1), 
the local upward tangential velocity component (due to counterclockwise rotation of the tornado) for the left half of cylinder 1 is larger than the right half, leading to a larger uplifting force exerted in the left half of the cylinder than that for the right half. This yields the overall clockwise twisting effect at point (A).

An inspection of $C_{m}$ curve of cylinder 2 opens up a global maximum at point (B) of Figure 5.3. With the Figure 5.9 showing cylinder 2 on the upper right portion of the core of the tornado, it is expected to experience clockwise moment. But due to the interference of cylinders 1 and 3 , an anticlockwise moment of higher magnitude is been produced on cylinder 2 at point $(B)$. The $C_{m}$ curve of cylinder 7 displays a local minimum point marked as (C). With the Figure 5.10, corresponding to point $(\mathbb{C}$, showing the cylinder 7 in the lower right core region of the tornado, an anticlockwise moment is been created as expected as per Eq. (2.1). 


\section{Chapter 6 - Summary of the Numerical Results for Case A to G}

\subsection{Tabulation of Case A Results}

After the detailed study on test case A, the numerical values of the maximum and minimum aerodynamic coefficients obtained on each cylinder of case A have been summarized and the case A, as already mentioned, corresponds to the orientation angle of $180^{\circ}$. Table 6.1 clearly affirms that the cylinder 1 is the most affected one experiencing both the highest magnitudes of force and moment coefficients. It also indicates that the cylinder 2 holds the second highest magnitudes of the same coefficients. A careful examination of the numbers tells that the force on cylinder 1 is more than twice on cylinder 2 and almost 9 times bigger than cylinder 5, which experiences the least force coefficient. Since the tornado moves towards the upper half of the model due to its anticlockwise rotation, the wind-loadings are higher on the top 3 cylinders in the case A orientation, i.e., cylinders 1, 2, and 3. But, the cylinder 7, which is at bottom, also experiences higher coefficients because it is exposed to tornado's unperturbed velocity pattern.

Table 6.1 Summary of Wind - Loading Coefficients for case A

\begin{tabular}{|c|c|c|c|}
\hline Cylinder & $\boldsymbol{C}_{\boldsymbol{F}}$ Maximum & $\boldsymbol{C}_{\boldsymbol{m}}$ Maximum & $\boldsymbol{C}_{\boldsymbol{m}}$ Minimum \\
\hline Cylinder 1 & 88.8559 & 3.5600 & -11.0728 \\
\hline Cylinder 2 & 24.8198 & 3.9407 & -3.2458 \\
\hline Cylinder 3 & 27.8759 & 1.7370 & -3.5837 \\
\hline Cylinder 4 & 12.7055 & 0.7059 & -0.8964 \\
\hline Cylinder 5 & 10.4635 & 0.7471 & -1.1765 \\
\hline Cylinder 6 & 12.5392 & 0.9629 & -1.4437 \\
\hline Cylinder 7 & 36.7258 & 3.1099 & -5.1005 \\
\hline
\end{tabular}




\subsection{Tabulation of Case B Results}

The numerical values of the wind-loading coefficients of case B, which has the orientation angle of $150^{\circ}$, have been encapsulated in the Table 6.2 It is obvious from the numbers that the cylinder 1 again experiences the highest values of the force and moment coefficients. But, this time, the cylinder 2 becomes the second most affected as opposed to cylinder 7 in case A. Further, the force coefficients on the top 3 cylinders exhibit higher values compared to case A. In particular, the maximum force coefficient that occurs on cylinder 1 has shown a tremendous increase of $15 \%$ approximately.

Table 6.2 Summary of Wind - Loading Coefficients for case B

\begin{tabular}{|c|c|c|c|}
\hline Cylinder & $\boldsymbol{C}_{\boldsymbol{F}}$ Maximum & $\boldsymbol{C}_{\boldsymbol{m}}$ Maximum & $\boldsymbol{C}_{\boldsymbol{m}}$ Minimum \\
\hline Cylinder 1 & 102.6730 & 4.3316 & -12.2445 \\
\hline Cylinder 2 & 45.2495 & 3.7761 & -6.7055 \\
\hline Cylinder 3 & 30.7134 & 1.5234 & -4.8149 \\
\hline Cylinder 4 & 16.0812 & 2.6964 & -2.2625 \\
\hline Cylinder 5 & 10.0977 & 0.9218 & -1.7163 \\
\hline Cylinder 6 & 7.0389 & 0.4312 & -1.8488 \\
\hline Cylinder 7 & 18.7627 & 2.1354 & -3.3987 \\
\hline
\end{tabular}

Figure 6.1 and Figure 6.2 represent the velocity magnitude contours at time instances when the total force coefficients on cylinder 1 peaked for the cases A and B respectively. An investigation on both the figures reveals the fact that cylinder 1 in case $\mathrm{B}$, corresponding to Figure 6.2, has higher velocity in its front portion compared to cylinder 1 in case A, corresponding to 
Figure 6.1 This difference is mainly due to two factors; the undistorted velocity pattern of the tornado in case B, relative to case A, when the cylinder 1 achieved its maximum force coefficient; reduced interference effects of cylinder 7 on cylinder 1 in the event of case B. The above-stated two factors can be indubitably ascribed to the decrease in the orientation angle from $180^{\circ}$ to $150^{\circ}$. The $30^{\circ}$ decrease in the orientation angle has completely altered the flow features of the tornado's interaction with the individual cylinders as well their interference effects on one another. Therefore, the decrease in the orientation angle initially from case A to case B has produced an increase in the magnitudes of the force as well as moment coefficients. The remaining test cases will explore the characteristics of the coefficients by further decreasing the orientation angle until it reaches $0^{\circ}$ in case $\mathrm{G}$.

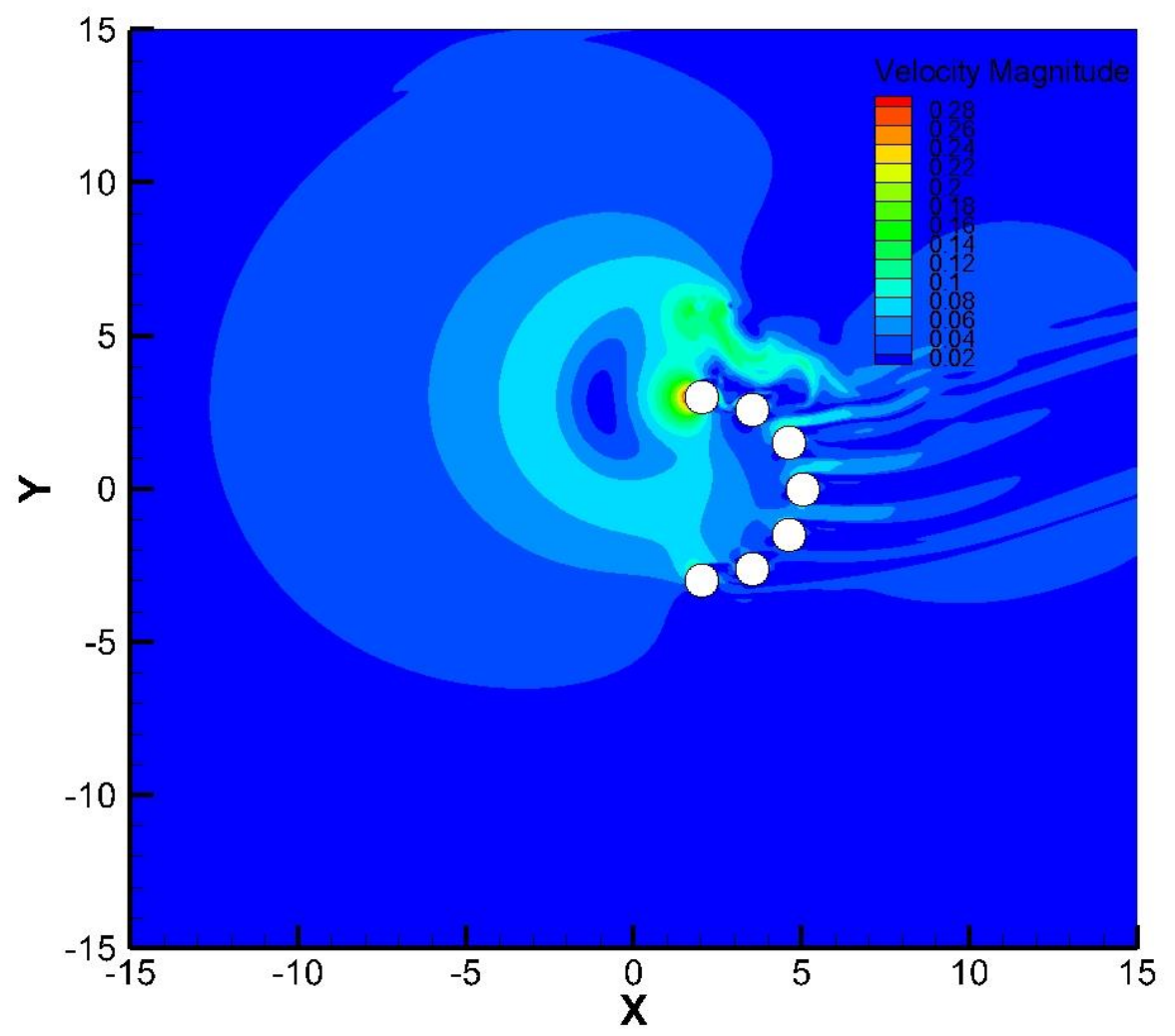

Figure 6.1 Velocity magnitude contour at $\left(C_{F}\right)_{\max }$ on cylinder 1 for case $A$ 


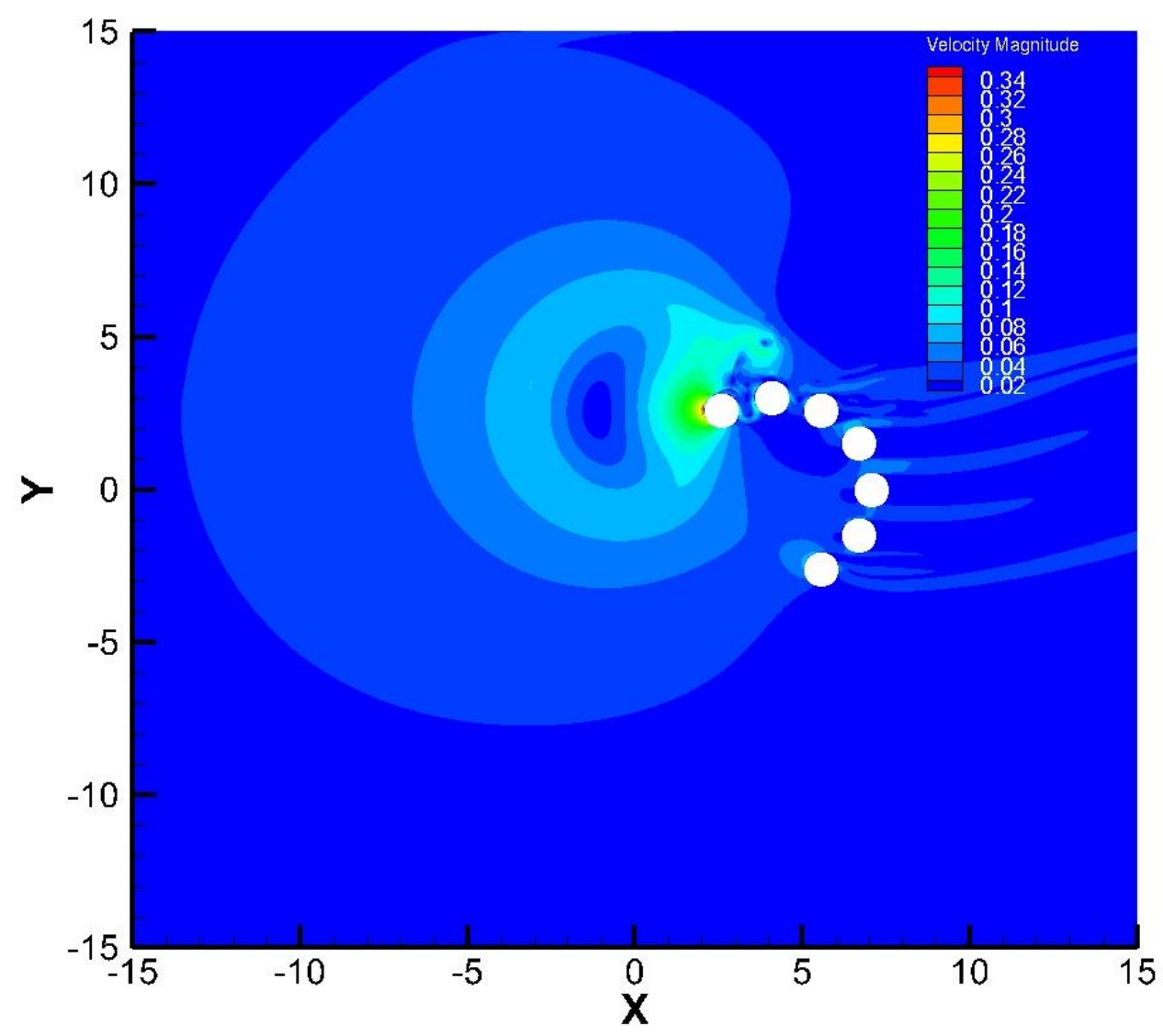

Figure 6.2 Velocity magnitude contour at $\left(C_{F}\right)_{\max }$ on cylinder 1 for case $B$

\subsection{Tabulation of Case C Results}

Table 6.3 summarizes the numerical values of the coefficients on each cylinder of case $\mathrm{C}$ that pertains to the orientation angle of $120^{\circ}$. From the data available in Table 6.3 it is found that the maximum force coefficient persists with the cylinder 1, despite another decrease in the orientation angle of $30^{\circ}$. But the value of maximum force coefficient on cylinder 1 is lower compared to both the cases A and B. As far as the maximum force coefficient on cylinder 1 is concerned, it initially increased (case A to B) and then decreased (case B to C) when the orientation angle was decreased from $180^{\circ}$ to $120^{\circ}$. Similar to case A and B, the top 3 cylinders again experiences higher force coefficients compared to the rest of the cylinders. An interesting trend that can be observed in the 
cases $\mathrm{B}$ and $\mathrm{C}$ is that the maximum force coefficient decreases from cylinder 1 to 6 and then increases on the cylinder 7.

Table 6.3 Summary of Wind - Loading Coefficients for case C

\begin{tabular}{|c|c|c|c|}
\hline Cylinder & $\boldsymbol{C}_{\boldsymbol{F}}$ Maximum & $\boldsymbol{C}_{\boldsymbol{m}}$ Maximum & $\boldsymbol{C}_{\boldsymbol{m}}$ Minimum \\
\hline Cylinder 1 & 81.7222 & 4.3647 & -10.1254 \\
\hline Cylinder 2 & 59.5170 & 2.6335 & -13.5647 \\
\hline Cylinder 3 & 38.8101 & 3.4255 & -8.9511 \\
\hline Cylinder 4 & 25.6097 & 4.5111 & -5.9899 \\
\hline Cylinder 5 & 18.6537 & 4.7871 & -6.4698 \\
\hline Cylinder 6 & 15.6025 & 7.7926 & -5.9221 \\
\hline Cylinder 7 & 18.0256 & 1.9169 & -1.9828 \\
\hline
\end{tabular}

Turning to the consideration of the moment coefficient, the greatest magnitude occurs on the cylinder 2, as opposed to cylinder 1 in the cases A and B. It is important to realize that the greatest magnitude has not only jumped from cylinder 1 to cylinder 2, but also achieved a higher value in comparison to the first two test cases A and B. Further, the magnitude of the moment coefficient on cylinder 2 has shown an enormous increase of $102 \%$ approximately from case B to case C, i.e., for an increase of $30^{\circ}$ orientation angle.

Figure 6.3 and Figure 6.4 represent the velocity contours that correspond to time instances when the cylinder 2 achieves its highest magnitude of moment coefficients for the cases B and C respectively. As per Eq. (2.1), the local upward tangential velocity on the left portion of cylinder 2 is higher than on the right portion and therefore a clockwise moment has been produced on 
cylinder 2 in both the cases. But the clockwise moment produced in the event of case $\mathrm{C}$ is higher, for the difference in orientation angle has modified the amount of interference caused by cylinder 1 on cylinder 2. The cylinder 1 in Figure 6.4 is seen to be obstructing more core flow from the bottom region of the tornado than in Figure 6.3. Consequently, a large local region of low velocities can be apparently seen on the right portion of the cylinder 2 in Figure 6.4 and this leads to the higher clockwise moment in case $\mathrm{C}$.

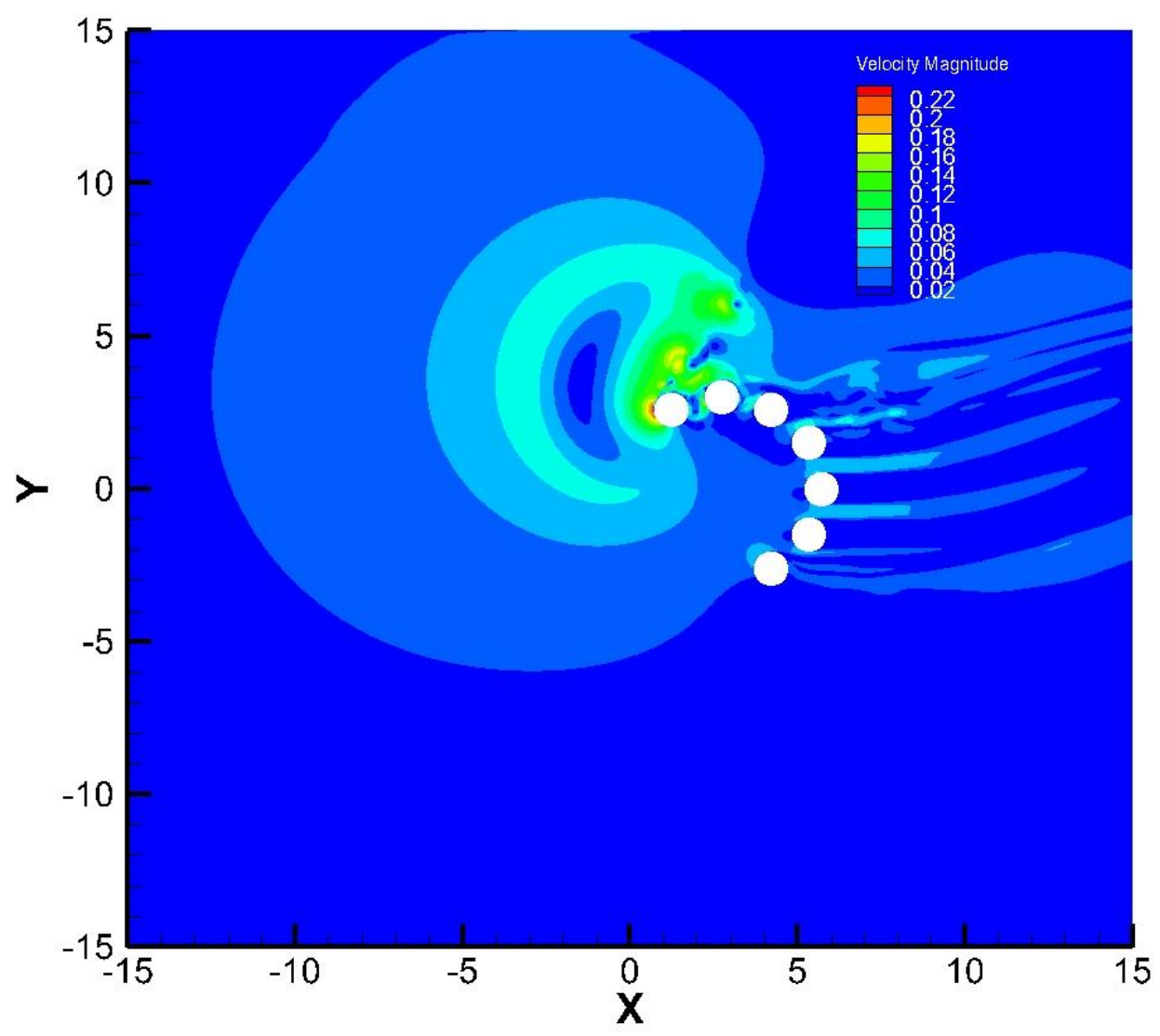

Figure 6.3 Velocity magnitude contour at 1) $\left(C_{m}\right)_{\min }$ on cylinder 2 for case $\left.B 2\right)\left(C_{F}\right)_{\max }$ on cylinder 1 for case $C$ 


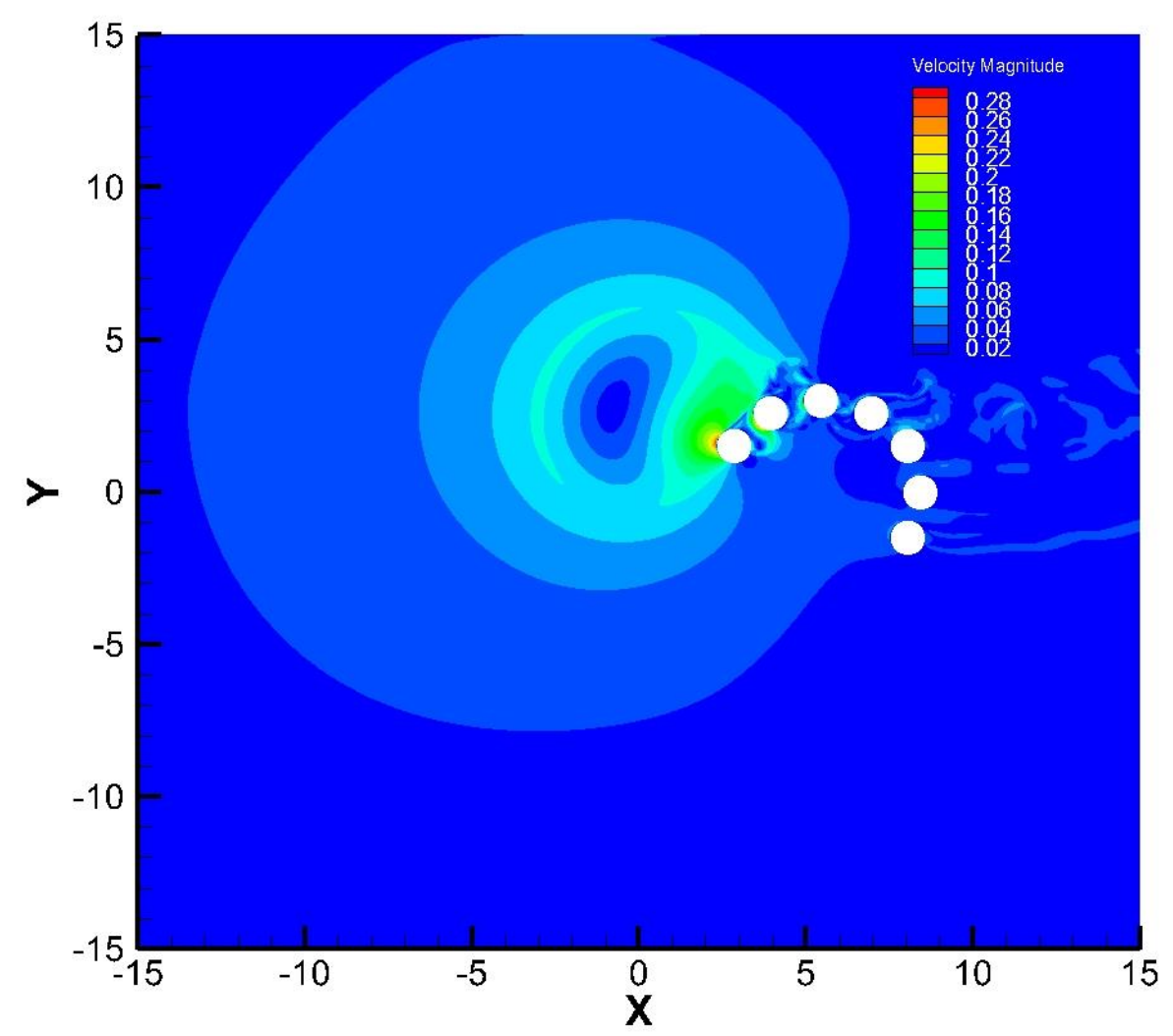

Figure 6.4 Velocity magnitude contour at $\left(C_{m}\right)_{\min }$ on cylinder 2 for case $C$

\subsection{Tabulation of Case $D$ Results}

Table 6.4 summarizes the numerical values of the wind-loading coefficients on each cylinder of case $\mathrm{D}$ that has the orientation angle of $90^{\circ}$. It clearly shows that the maximum force coefficient has been shifted to cylinder 2, as opposed to cylinder 1 in all the previous three cases A to C. But, the magnitude of the force coefficient has achieved a lower value when compared to the cases A to C. For a decrease in orientation angle from $120^{\circ}$ to $90^{\circ}$, the maximum force coefficient has exhibited a massive decrease of $25 \%$. It is also noted that the moment coefficient of greatest magnitude has been displaced from cylinder 2, as in case $\mathrm{C}$, to cylinder 3 and has shown a slight decrease. 
Table 6.4 Summary of Wind - Loading Coefficients for case D

\begin{tabular}{|c|c|c|c|}
\hline Cylinder & $\boldsymbol{C}_{\boldsymbol{F}}$ Maximum & $\boldsymbol{C}_{\boldsymbol{m}}$ Maximum & $\boldsymbol{C}_{\boldsymbol{m}}$ Minimum \\
\hline Cylinder 1 & 48.8437 & 3.4320 & -7.3155 \\
\hline Cylinder 2 & 61.5281 & 3.2932 & -6.2029 \\
\hline Cylinder 3 & 46.1234 & 2.2248 & -11.9023 \\
\hline Cylinder 4 & 52.0965 & 2.1562 & -9.7535 \\
\hline Cylinder 5 & 34.4174 & 2.9705 & -7.6407 \\
\hline Cylinder 6 & 21.8735 & 6.6159 & -7.2873 \\
\hline Cylinder 7 & 21.4915 & 8.0970 & -6.1180 \\
\hline
\end{tabular}

Figure 6.3 and Figure 6.5 represent the velocity magnitude contours which correspond to $\left(C_{F}\right)_{\max }$ on cylinders 1 and 2 for the test cases $C$ and D respectively. As per Eq. (2.1) and (2.5), the tangential velocity, $V_{\gamma}$, is inversely proportional to the radial distance from the centre of the core of the tornado. The cylinder 2 in Figure 6.5 is closer to the core than the cylinder 1 in Figure 6.3 and hence higher force coefficient is expected on cylinder 2. But the lesser orientation angle in case $\mathrm{D}$ has reformed the flow around cylinder 2 by improving the interference effects of cylinder 1 on cylinder 2. Also, the concurrent translation and counterclockwise rotation moves the core of the tornado more towards the cylinder 3 than cylinder 2 and thereby creates a lesser maximum force coefficient on cylinder 2. It is important to note that the cylinders that are exposed to core of the tornado, cylinders 1 to 4, experience the higher force coefficients. It is clearly evident that the change in orientation angle, besides modifying the maximum force coefficient, brings about the change in the force coefficients on all the cylinders in the model. 


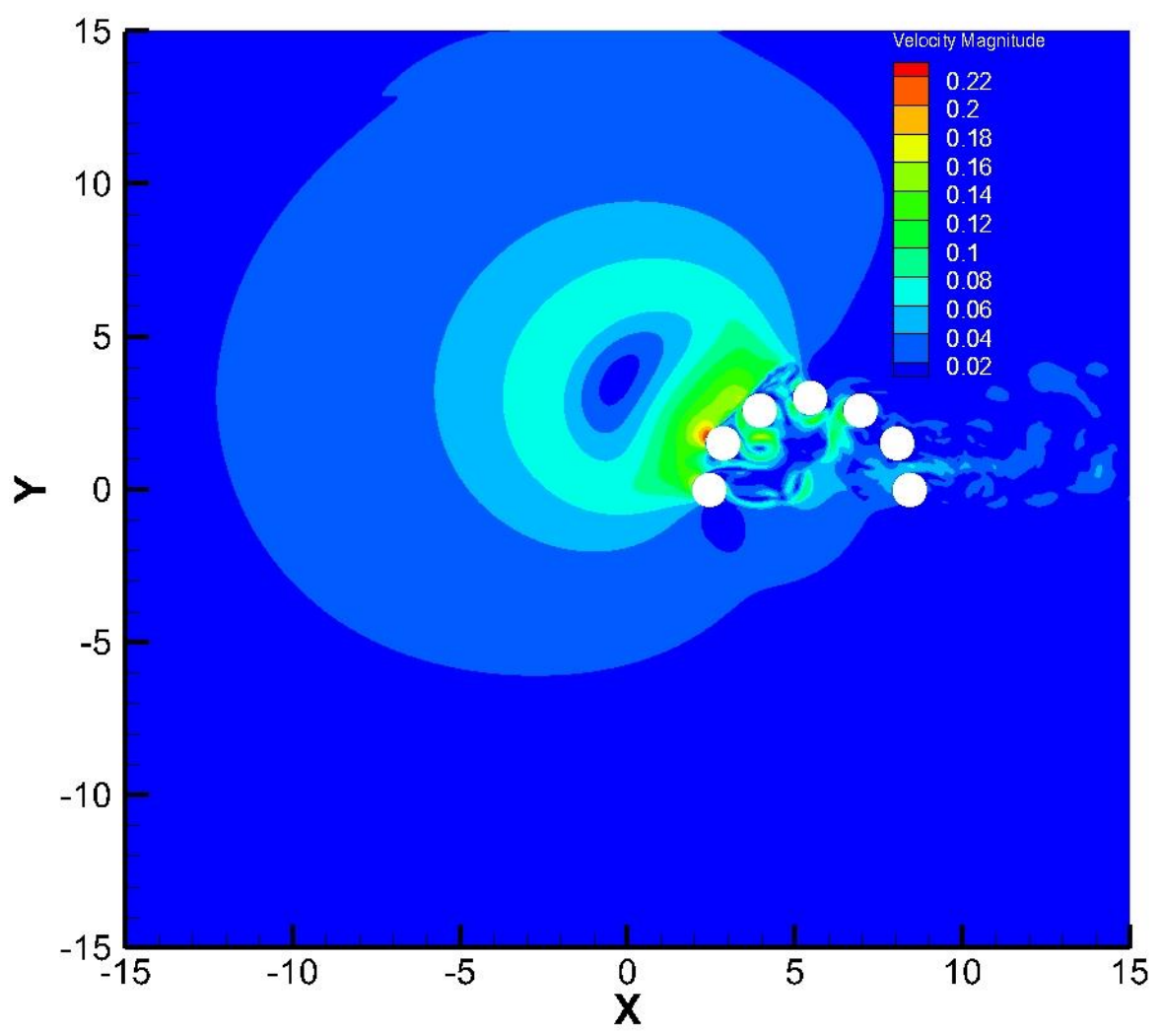

Figure 6.5 Velocity magnitude contour at $\left(C_{F}\right)_{\max }$ on cylinder 2 for case $D$

\subsection{Tabulation of Case E Results}

Table 6.5 gives the summary of the numerical values of the wind-loading coefficients of each cylinder of case $\mathrm{E}$ which pertains to the orientation angle of $60^{\circ}$. It can be easily seen that the maximum force coefficient has been transferred to occur on cylinder 3 , as opposed to cylinder 2 in case $\mathrm{D}$, with a slight decrease in magnitude. Again, the cylinders lying above the initial line of fire of the tornado, cylinders 2 to 5 , experience the higher force coefficients which is evident from the data in Table 6.5 Despite a decrease in the orientation angle of $30^{\circ}$ from case $\mathrm{D}$ to $\mathrm{E}$, the maximum force coefficient almost remains unchanged. Therefore, it is expected that the maximum force coefficient remains lower in the range of $60^{\circ}$ to $90^{\circ}$. 
Table 6.5 Summary of Wind - Loading Coefficients for case E

\begin{tabular}{|c|c|c|c|}
\hline Cylinder & $\boldsymbol{C}_{\boldsymbol{F}}$ Maximum & $\boldsymbol{C}_{\boldsymbol{m}}$ Maximum & $\boldsymbol{C}_{\boldsymbol{m}}$ Minimum \\
\hline Cylinder 1 & 15.9242 & 2.0148 & -3.4926 \\
\hline Cylinder 2 & 48.8331 & 2.7955 & -6.7715 \\
\hline Cylinder 3 & 61.0558 & 3.6812 & -5.8865 \\
\hline Cylinder 4 & 35.6085 & 2.7397 & -7.7835 \\
\hline Cylinder 5 & 30.3534 & 1.9423 & -7.2055 \\
\hline Cylinder 6 & 19.2962 & 3.7754 & -5.5305 \\
\hline Cylinder 7 & 25.9808 & 5.8935 & -8.3162 \\
\hline
\end{tabular}

As far as the moment coefficient is concerned, the occurrence of greatest magnitude has moved from cylinder 3 to cylinder 7 from case D to E. Also, it has shown a decrease of $30 \%$ approximately for a decrease of $30^{\circ}$ in the orientation angle. Figure 6.6 and Figure 6.7 depict the velocity magnitude contours that correspond to greatest moment coefficient on cylinders 3 and 7 for the test cases D and E respectively. The decrease in moment coefficient on cylinder 7 of case E is principally due the completely distorted velocity pattern of the tornado observed in Figure 6.7 produces the lower upward tangential velocity on the left portion of cylinder 7 compared to the higher upward tangential velocity on the left portion of cylinder 3 in Figure 6.6. The distorted velocity pattern in Figure 6.7 is primarily created by the interference effects of the cylinders 1,2 , and 3 on cylinder 7 , which in turn is attributed mainly to the decrease in the orientation angle from $90^{\circ}$ to $60^{\circ}$. 


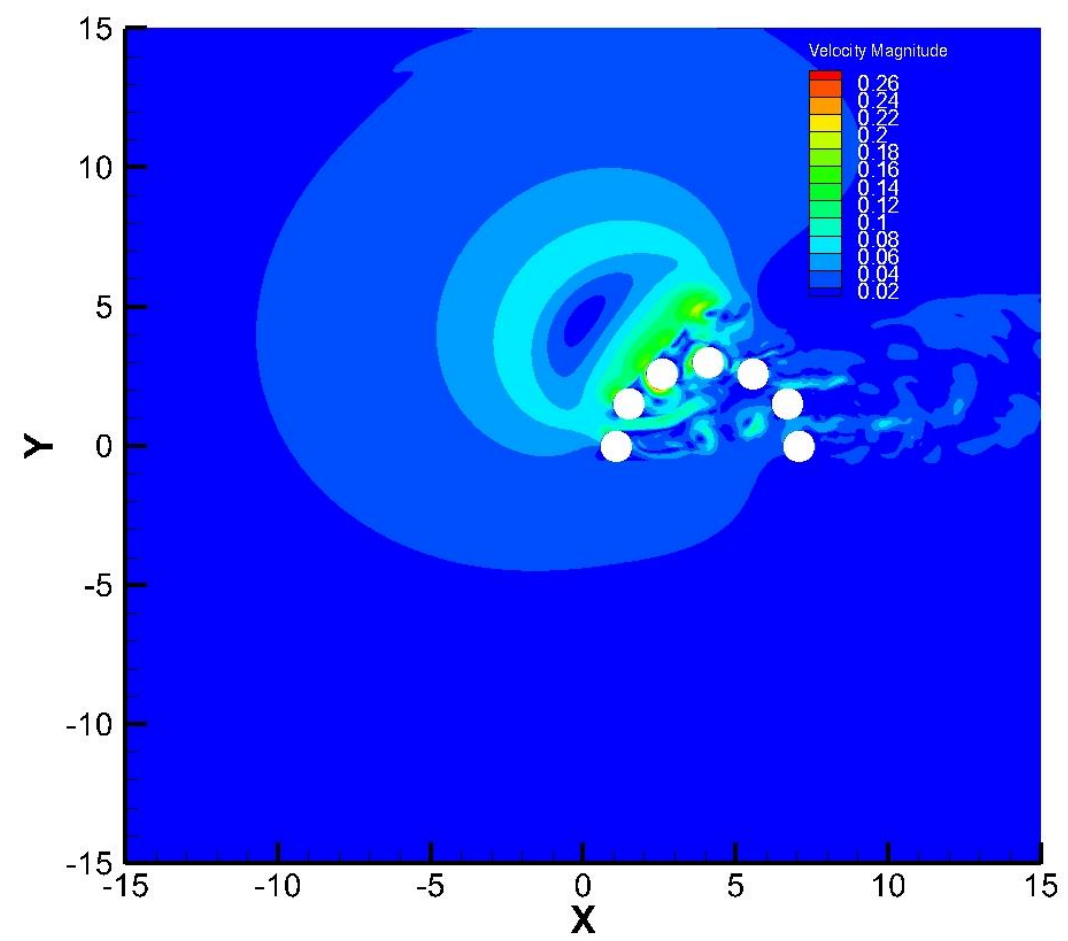

Figure 6.6 Velocity magnitude contour at $\left(C_{m}\right)_{\min }$ on cylinder 3 for case $D$

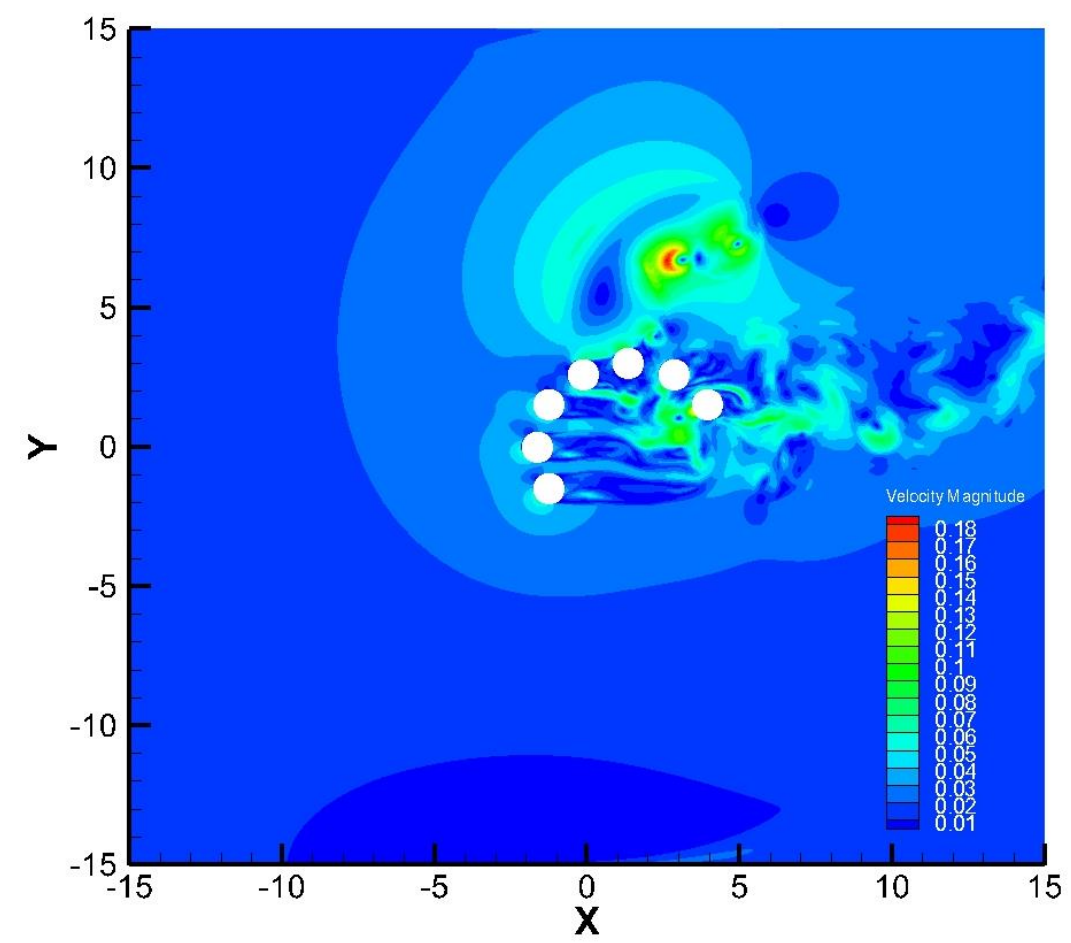

Figure 6.7 Velocity magnitude contour at $\left(C_{m}\right)_{\min }$ on cylinder 7 for case $E$ 


\subsection{Tabulation of Case F Results}

The numerical values of the coefficients on each cylinder of case $F$ have been summarized in the Table 6.6 and case F corresponds to the orientation angle of $30^{\circ}$. It clearly indicates that the value of the maximum force coefficient has undergone a slight change, but it has been moved to occur on cylinder 4 rather than cylinder 3, as in the previous case E. It is important to note that the cylinders which lie above the initial line of fire of the tornado, cylinders 3,4 , and 5, experience the higher force coefficients since they pass through the core of the tornado. Regarding the greatest moment coefficient, a particular trend is not observed while decreasing the orientation angle every $30^{\circ}$. From case $\mathrm{E}$ to $\mathrm{F}$, the greatest moment coefficient has moved from cylinder 7 to cylinder 5 without any appreciable change. Despite the decrease in the orientation angle of $30^{\circ}$, neither the maximum force coefficient nor the greatest moment coefficient shows any substantial change and this can be explained with the help of the Figure 6.8 and Figure 6.9.

Table 6.6 Summary of Wind - Loading Coefficients for case F

\begin{tabular}{|c|c|c|c|}
\hline Cylinder & $\boldsymbol{C}_{\boldsymbol{F}}$ Maximum & $\boldsymbol{C}_{\boldsymbol{m}}$ Maximum & $\boldsymbol{C}_{\boldsymbol{m}}$ Minimum \\
\hline Cylinder 1 & 6.4497 & 0.6877 & -2.2259 \\
\hline Cylinder 2 & 17.8142 & 1.6493 & -3.4297 \\
\hline Cylinder 3 & 49.8475 & 2.8567 & -6.8275 \\
\hline Cylinder 4 & 60.3844 & 3.7586 & -5.8721 \\
\hline Cylinder 5 & 37.9057 & 2.5845 & -8.0780 \\
\hline Cylinder 6 & 31.6175 & 1.9383 & -5.5579 \\
\hline Cylinder 7 & 16.3676 & 3.8797 & -2.6011 \\
\hline
\end{tabular}


Figure 6.8 and Figure 6.9 depict velocity magnitude contours at the time instances when the cylinders 3 and 4 achieve $\left(C_{F}\right)_{\max }$ for the cases $\mathrm{E}$ and $\mathrm{F}$ respectively. In both the figures, it is clear that the cylinders which lie above the initial line of fire of the tornado faces the core region of the tornado. It can be easily seen that the velocity magnitudes around the top 3 cylinders are essentially the same in both the depictions. Due to the right-upward path of the tornado, the interference effects of the cylinders below the line of fire, cylinder 1 and cylinders 1 and 2 in the case of $E$ and F respectively, on the top cylinders have produced no significant alterations in the flow field and thereby the Figure 6.8 and Figure 6.9 bear a complete resemblance to each other. The aforementioned factors lead to the same values of the maximum force coefficients for the cases $\mathrm{E}$ and $\mathrm{F}$, despite a considerable change in the orientation angle.

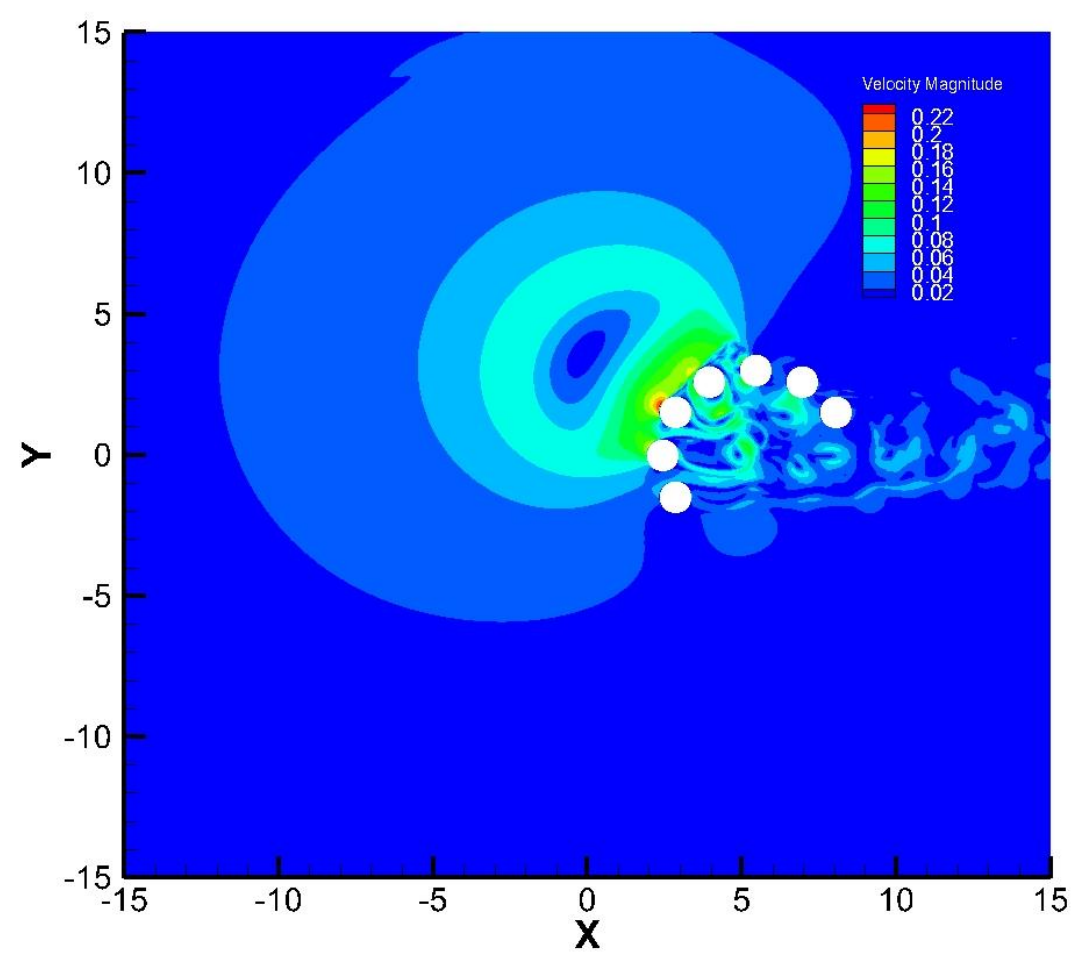

Figure 6.8 Velocity magnitude contour at $\left(C_{F}\right)_{\max }$ on cylinder 3 for case $E$ 


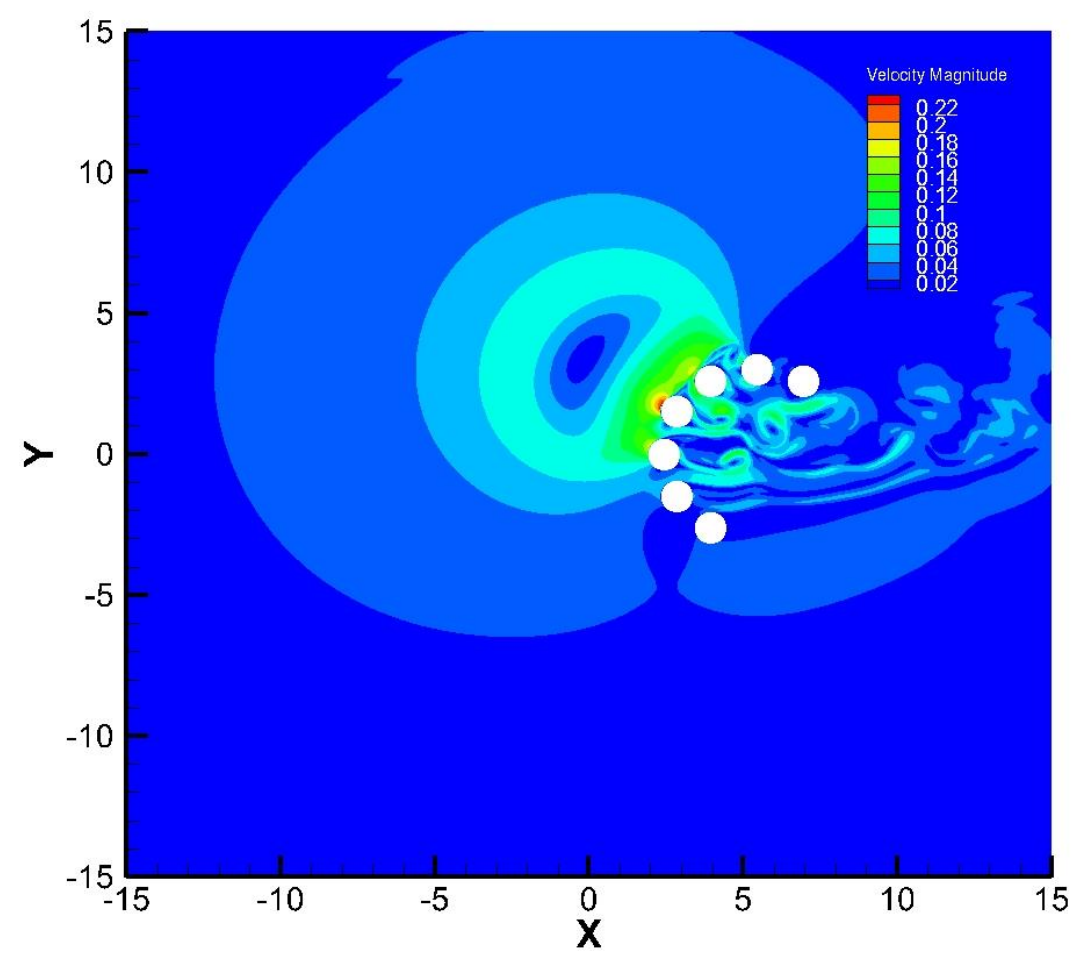

Figure 6.9 Velocity magnitude contour at $\left(C_{F}\right)_{\max }$ on cylinder 4 for case $F$

\subsection{Tabulation of Case G Results}

The numerical values of the wind-loadings on each cylinder of case $\mathrm{G}$ which corresponds to the orientation angle of $0^{\circ}$ has been tabulated and given in Table 6.7 . It is obvious that the maximum force coefficient has been progressed from cylinder 4, as in case $\mathrm{F}$, to cylinder 5 . Concerning the maximum force coefficient, a trend can be observed such that, for the first three test cases $\mathrm{A}$ to $\mathrm{C}$, the cylinder 1 experiences the maximum force coefficient, and for the next four test cases $\mathrm{D}$ to $\mathrm{G}$, the maximum force coefficient progresses from one cylinder to another in the clockwise direction, i.e., from cylinder 2 to cylinder 5 for the four subsequent cases respectively. The highest magnitude of moment coefficient has jumped from cylinder 5, as in case F, to cylinder 6 with no noticeable change in its value. 
Table 6.7 Summary of Wind - Loading Coefficients for case G

\begin{tabular}{|c|c|c|c|}
\hline Cylinder & $\boldsymbol{C}_{\boldsymbol{F}}$ Maximum & $\boldsymbol{C}_{\boldsymbol{m}}$ Maximum & $\boldsymbol{C}_{\boldsymbol{m}}$ Minimum \\
\hline Cylinder 1 & 6.4011 & 0.6423 & -2.4604 \\
\hline Cylinder 2 & 7.0299 & 0.6242 & -2.2440 \\
\hline Cylinder 3 & 18.8910 & 1.5865 & -3.5952 \\
\hline Cylinder 4 & 50.5740 & 2.7057 & -6.8752 \\
\hline Cylinder 5 & 61.0870 & 3.3294 & -5.8842 \\
\hline Cylinder 6 & 38.1875 & 1.7919 & -8.2213 \\
\hline Cylinder 7 & 24.3360 & 5.0154 & -7.5701 \\
\hline
\end{tabular}

\subsection{The Unluckiest or Most Affected Building}

In this section, the building which is at the highest risk in the tornado is going to be found. Table 6.8 has been created from the data depicted from Table 6.1 to Table 6.7 and it shows the most affected cylinders of the test cases A to G. The rule of thumb used here to find the unluckiest building is that, the more the frequency of the cylinder number in Table 6.8, the higher the risk the cylinder faces. By that rule, the cylinders 1 and 7 appear seven and six times respectively in the table and it tells that the buildings at the extreme ends of the arrangement encounter greater damages in the tornado flow. Strictly speaking, the cylinder 1 is at the greatest risk in the arrangement as per the rule of thumb. The physical significance of this result is that the cylinder 1 is exposed to higher wind-loadings for just less than fifty percent of the total range of the orientation angles. 
Table 6.8 The Most affected Cylinders of the test cases A to G

\begin{tabular}{|c|c|c|c|}
\hline Case & $\boldsymbol{C}_{\boldsymbol{F}}$ Maximum & $\boldsymbol{C}_{\boldsymbol{m}}$ Maximum & $\boldsymbol{C}_{\boldsymbol{m}}$ Minimum \\
\hline $\mathrm{A}$ & 1 & 2 & 1 \\
\hline $\mathrm{B}$ & 1 & 1 & 1 \\
\hline $\mathrm{C}$ & 1 & 6 & 2 \\
\hline $\mathrm{D}$ & 2 & 7 & 7 \\
\hline $\mathrm{E}$ & 3 & 7 & 5 \\
\hline $\mathrm{F}$ & 4 & 7 & 6 \\
\hline $\mathrm{G}$ & 5 & 7 & \\
\hline
\end{tabular}




\section{Chapter 7 - Optimization Procedure}

\subsection{An Overview of Optimization Procedure Using Polynomial Fitting}

This section basically deals with two aspects that are mentioned as follows: a) polynomials of order 6,7 , and 8 have been fitted to each of the coefficients, $\left(C_{F}\right)_{\max },\left(C_{m}\right)_{\max }$, and $\left(C_{m}\right)_{\min }$ as a function of orientation angle for the unluckiest building, cylinder $1, \mathrm{~b}$ ) polynomials of order 6 , 7, and 8 have been fitted to each of the coefficients, $\left(C_{F}\right)_{\max },\left(C_{m}\right)_{\max }$, and $\left(C_{m}\right)_{\min }$ as a function of orientation angle in the overall sense, i.e. the maximum and minimum coefficients are chosen irrespective of the cylinders on which those values occur. The values of the coefficients have been taken from the tables provided in Chapter 8 and the polynomials have been fitted using "Lagrangian Interpolation Formula".

\subsection{Optimization of Cylinder 1}

\subsubsection{Polynomial for $\left(C_{F}\right)_{\max }$ on Cylinder 1}

Table 7.1 represents the variation of $\left(C_{F}\right)_{\max }$ of cylinder 1 with the orientation angle. In Table 7.1 , the first seven values are obtained from the seven test cases A to $G$ and the procurement and usage of the next two values will be explained later in this section.

Table 7.1 Variation of $\left(C_{F}\right)_{\max }$ with $\theta$ for cylinder 1

\begin{tabular}{|c|c|c|c|c|c|c|c|c|c|}
\hline $\begin{array}{c}\text { Orientation } \\
\text { angle } \boldsymbol{\theta} \\
(\mathbf{r a d})\end{array}$ & 0 & 0.5236 & 1.0472 & 1.5708 & 2.0944 & 2.6180 & 3.1416 & 0.633 & 0.155 \\
\hline$\left(\boldsymbol{C}_{\boldsymbol{F}}\right)_{\max }$ & 6.4011 & 6.4497 & 15.9242 & 48.8437 & 81.7222 & 102.6730 & 88.8559 & 7.3245 & 4.8077 \\
\hline
\end{tabular}

Using the first seven values in Table 7.1 , the polynomial of sixth degree for $\left(C_{F}\right)_{\max }$ of cylinder 1 has been found and given by: 


$$
\begin{aligned}
\left(C_{F}\right)_{\max } & =-4.8329 \theta^{6}+48.3594 \theta^{5}-187.8819 \theta^{4}+337.5149 \theta^{3} \\
& -245.2826 \theta^{2}+59.5166 \theta+6.4011
\end{aligned}
$$

In Eq. (7.1) as well as the all polynomial equations, the orientation angle $\theta$ is expressed in terms of radians. The polynomial curve given in Eq. (7.1) has been represented graphically in Figure 7.1.

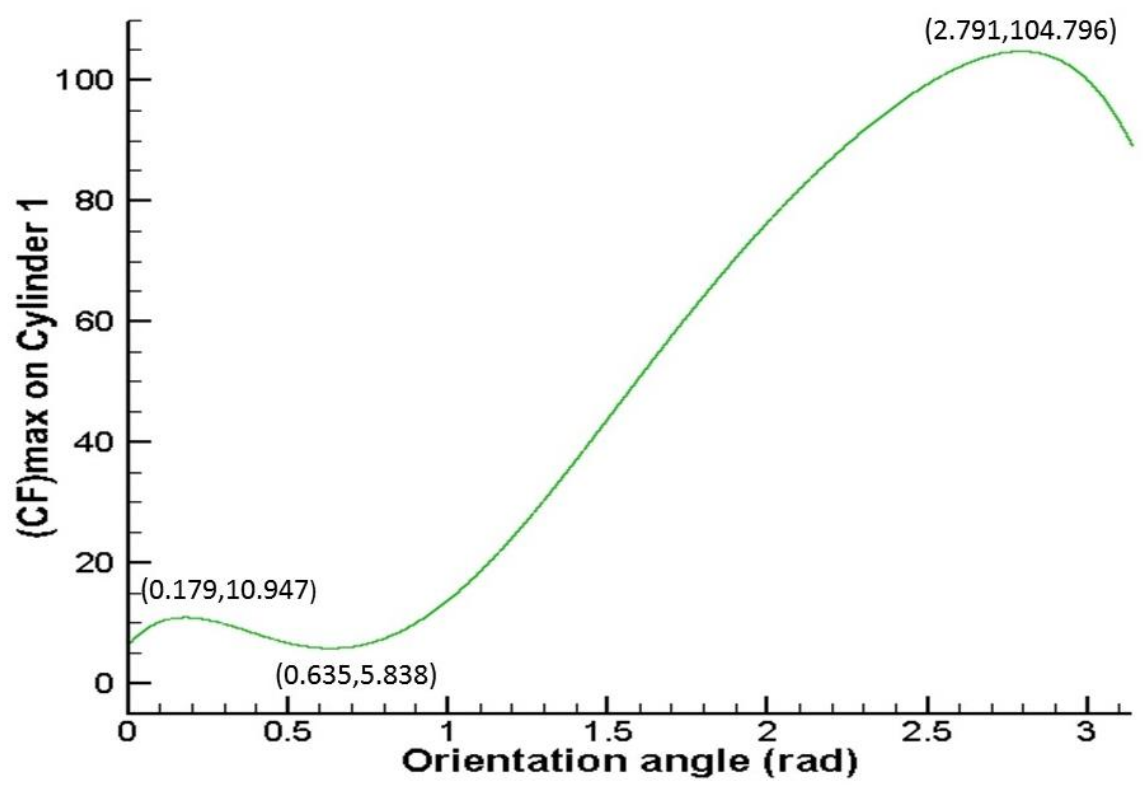

Figure 7.1 Variation of $6^{\text {th }}$ order $\left(C_{F}\right)_{\max }$ polynomial of cylinder 1 with $\theta$

The turning points marked in Figure 7.1 have been found by differentiating Eq. (7.1) with respect to $\theta$ and equating the first derivative to zero which is given below:

$$
\frac{d\left(C_{F}\right)_{\max }}{d \theta}=0
$$

Eq. (7.2) gives three stationary orientation angles. But the interest is on the value of the stationary orientation angle that has the minimum value of $\left(C_{F}\right)_{\max }$. The stationary orientation angle that has the minimum coefficient is $0.633 \mathrm{rad}$. In order to find the polynomials of seventh order, another 
data set is required and is found out by re-running the test case at $\theta=0.633 \mathrm{rad}$ and the obtained data set is included in Table 7.1 as $8^{\text {th }}$ data set. The mathematical polynomial of seventh order corresponding to $\left(C_{F}\right)_{\max }$ on cylinder 1 is given by Eq. (7.3) as below and its graphical form is represented in Figure 7.2.

$$
\begin{aligned}
\left(C_{F}\right)_{\max } & =-7.5911 \theta^{7}+78.6384 \theta^{6}-315.8687 \theta^{5}+613.1344 \theta^{4} \\
& -589.2269 \theta^{3}+281.8122 \theta^{2}-53.1352 \theta+6.4012
\end{aligned}
$$

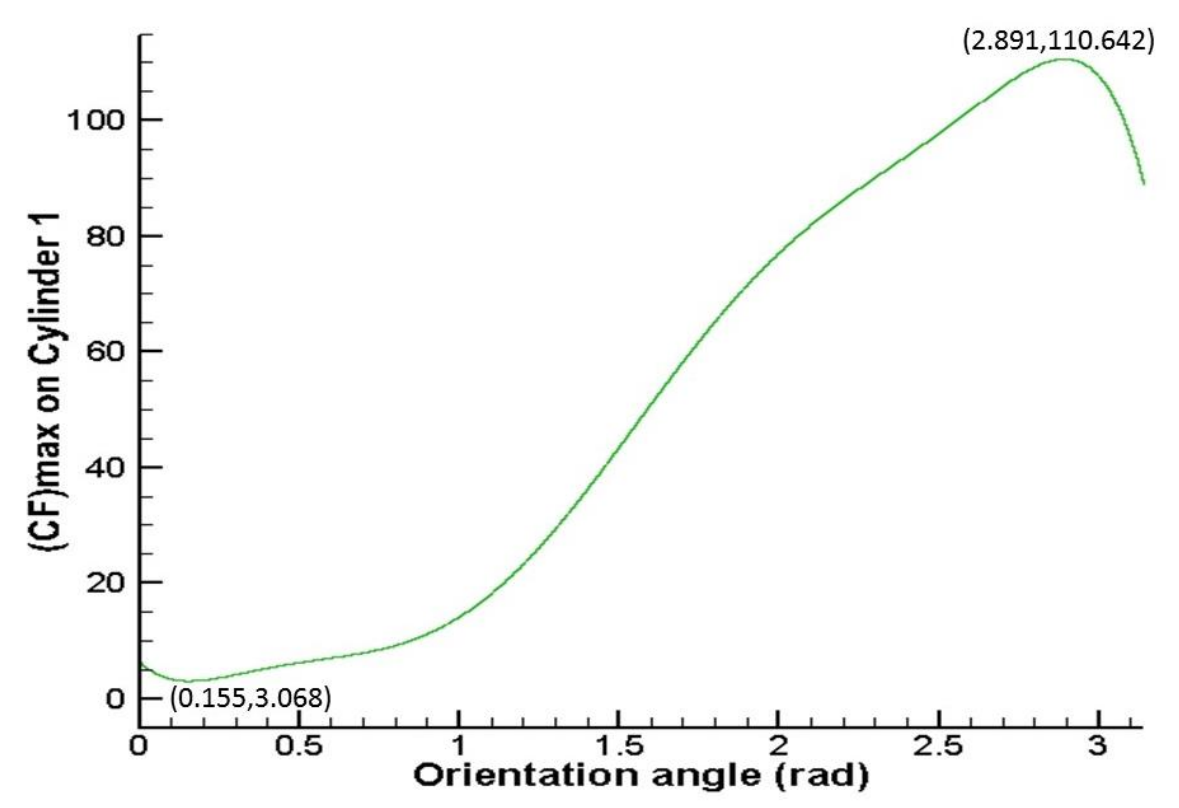

Figure 7.2 Variation of $7^{\text {th }}$ order $\left(C_{F}\right)_{\max }$ polynomial of cylinder 1 with $\theta$

The Figure 7.2 has again the turning points marked on it and the stationary orientation angle corresponding to minimum $\left(C_{F}\right)_{\max }$ is $0.155 \mathrm{rad}$. For the eight order polynomial to be found, the test has been re-run with the orientation angle to be $\theta=0.155 \mathrm{rad}$ and the data set has been added as $9^{\text {th }}$ data set. The polynomial of eighth order has been represented mathematically in Eq. (7.4) and graphically in Figure 7.3. 


$$
\begin{aligned}
\left(C_{F}\right)_{\max } & =-3.5343 \theta^{8}+33.5083 \theta^{7}-115.5301 \theta^{6}+164.3720 \theta^{5} \\
& -54.3309 \theta^{4}-70.7746 \theta^{3}+74.0617 \theta^{2}-19.9511 \theta+6.4029
\end{aligned}
$$

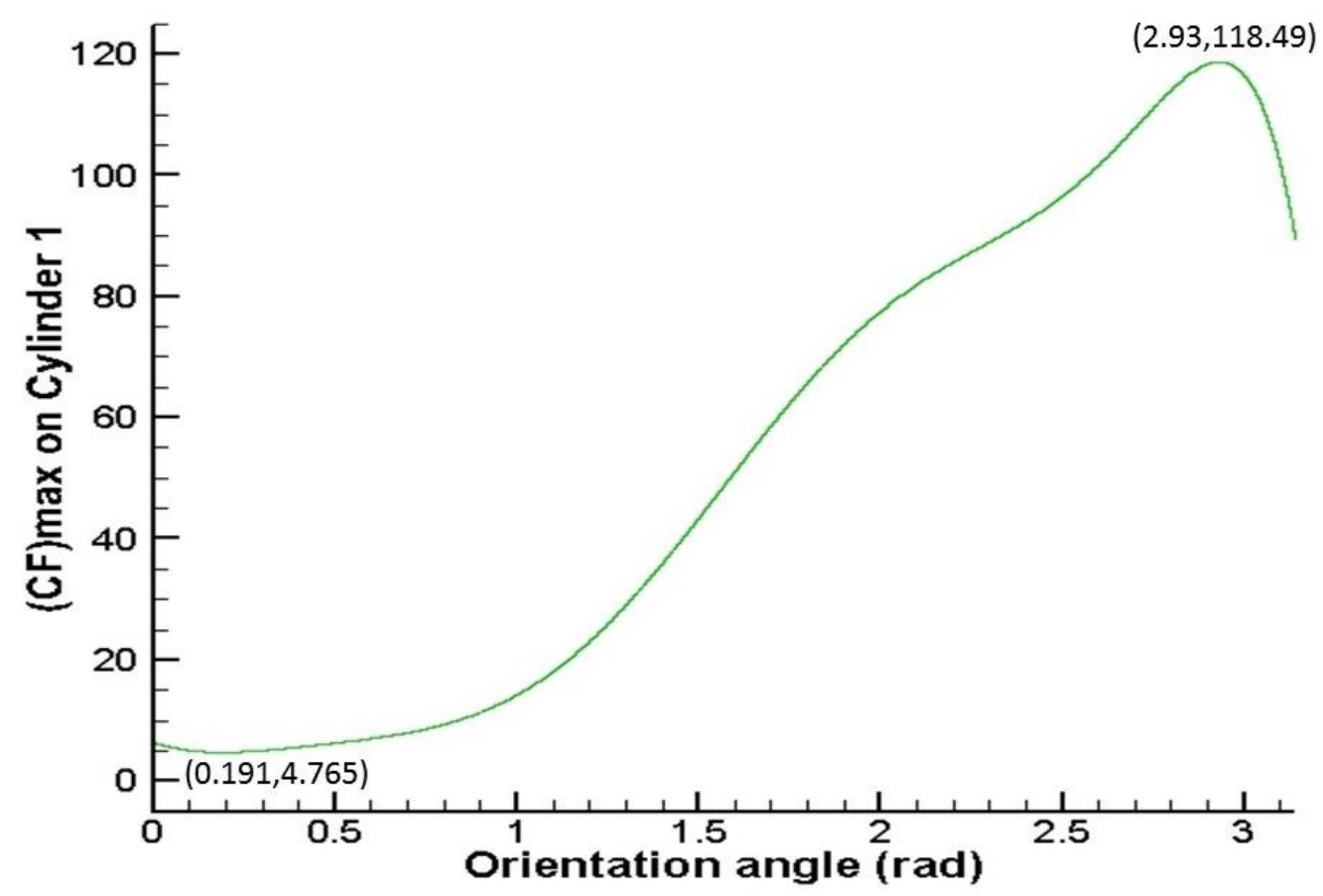

Figure 7.3 Variation of $8^{\text {th }}$ order $\left(C_{F}\right)_{\max }$ polynomial of cylinder 1 with $\theta$

An observation of the Figure 7.1, Figure 7.2, and Figure 7.3 reveal that the $\left(C_{F}\right)_{\max }$ curves follow a similar pattern of having oscillations initially and then increases monotonically to achieve the maximum and then decreases. It can be easily seen that the maximum value increases as the order of the polynomial increases. From the figures, a safe zone can be ascertained in which the value of $\left(C_{F}\right)_{\max }$ is acceptable and not very high. Looking at the figures, the safe zone can be defined from $\theta=0 \mathrm{rad}$ to $\theta=1.2 \mathrm{rad}$ where the value of the coefficient is below 20 that is relatively low compared to the rest of the region. 


\subsubsection{Polynomial for $\left(C_{m}\right)_{\max }$ on Cylinder 1}

Table 7.2 shows the values of $\left(C_{m}\right)_{\max }$ obtained for cylinder 1 at different values of orientation angles. As already mentioned in the previous section, the first seven data sets are used to find the polynomial of sixth order and the next two data sets are found in order to find the polynomials of seventh and eighth orders respectively.

Table 7.2 Variation of $\left(C_{m}\right)_{\max }$ with $\theta$ for cylinder 1

\begin{tabular}{|c|c|c|c|c|c|c|c|c|c|}
\hline $\begin{array}{c}\text { Orientation } \\
\text { angle } \boldsymbol{\theta} \\
(\mathbf{r a d})\end{array}$ & 0 & 0.5236 & 1.0472 & 1.5708 & 2.0944 & 2.6180 & 3.1416 & 0.222 & 0.285 \\
\hline$\left(\boldsymbol{C}_{\boldsymbol{m}}\right)_{\max }$ & 0.6423 & 0.6877 & 2.0148 & 3.4320 & 4.3647 & 4.3316 & 3.9407 & 0.5175 & 0.9315 \\
\hline
\end{tabular}

The polynomials of orders six, seven, and eight for $\left(C_{m}\right)_{\max }$ on cylinder 1 has been found and given by the Eq. (7.5), (7.6), and (7.7) respectively and the graphical representations of those equations are depicted in the Figure 7.4,Figure 7.5, and Figure 7.6 respectively.

$$
\begin{aligned}
\left(C_{m}\right)_{\max }= & 0.1022 \theta^{6}-0.9136 \theta^{5}+3.3033 \theta^{4}-6.8203 \theta^{3}+8.4404 \theta^{2} \\
& -2.8723 \theta+0.6423 \\
\left(C_{m}\right)_{\max }= & 0.1681 \theta^{7}-1.7462 \theta^{6}+7.1509 \theta^{5}-14.4291 \theta^{4}+13.6916 \theta^{3} \\
& -3.2240 \theta^{2}-0.3798 \theta+0.6423 \\
\left(C_{m}\right)_{\max } & =8.3942 \theta^{8}-93.9947 \theta^{7}+421.4767 \theta^{6}-967.9125 \theta^{5} \\
& +1206.8086 \theta^{4}-796.5153 \theta^{3}+250.6846 \theta^{2}-28.0284 \theta \\
& +0.6424
\end{aligned}
$$




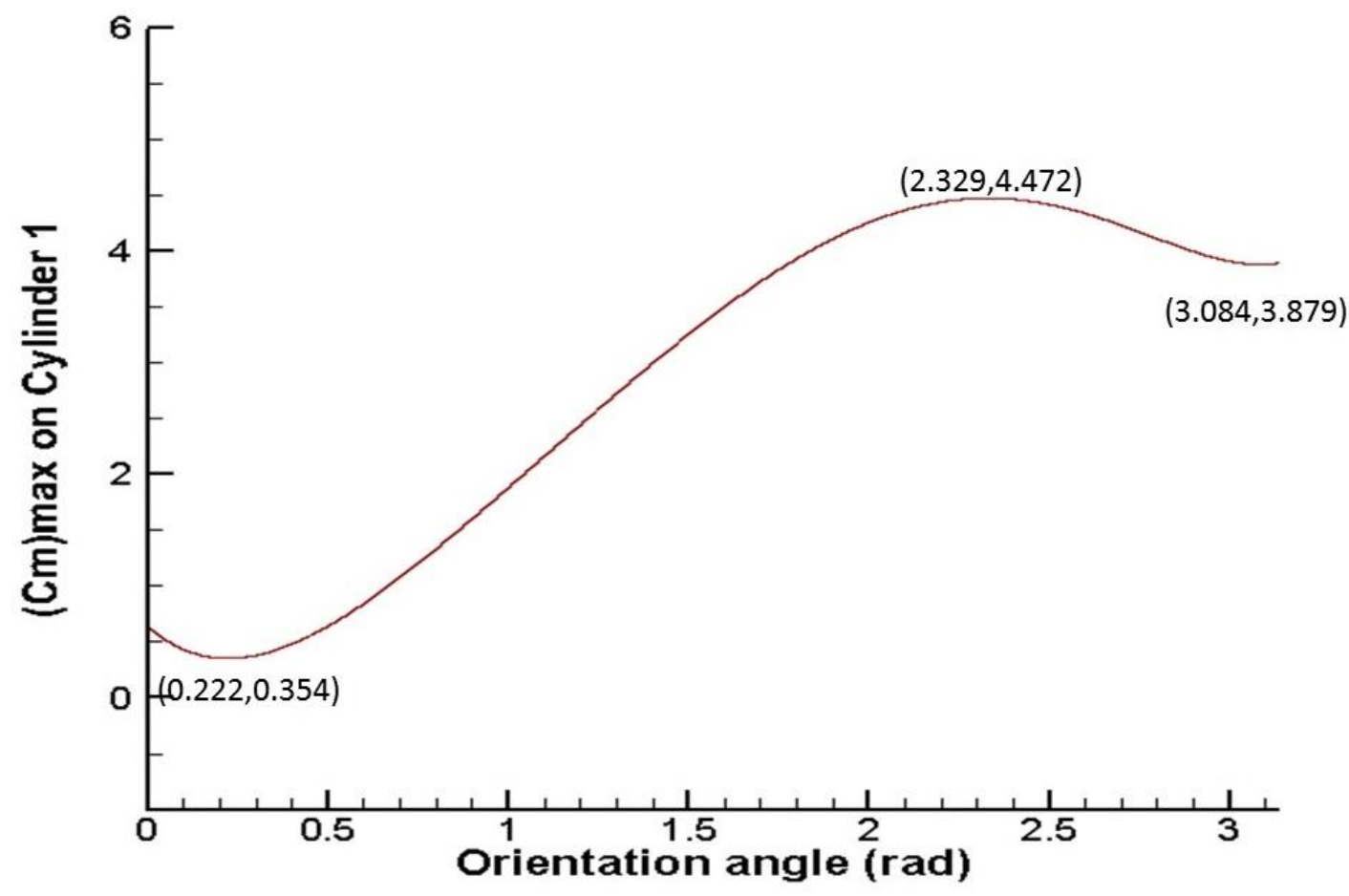

Figure 7.4 Variation of $6^{\text {th }}$ order $\left(C_{m}\right)_{\max }$ polynomial of cylinder 1 with $\theta$

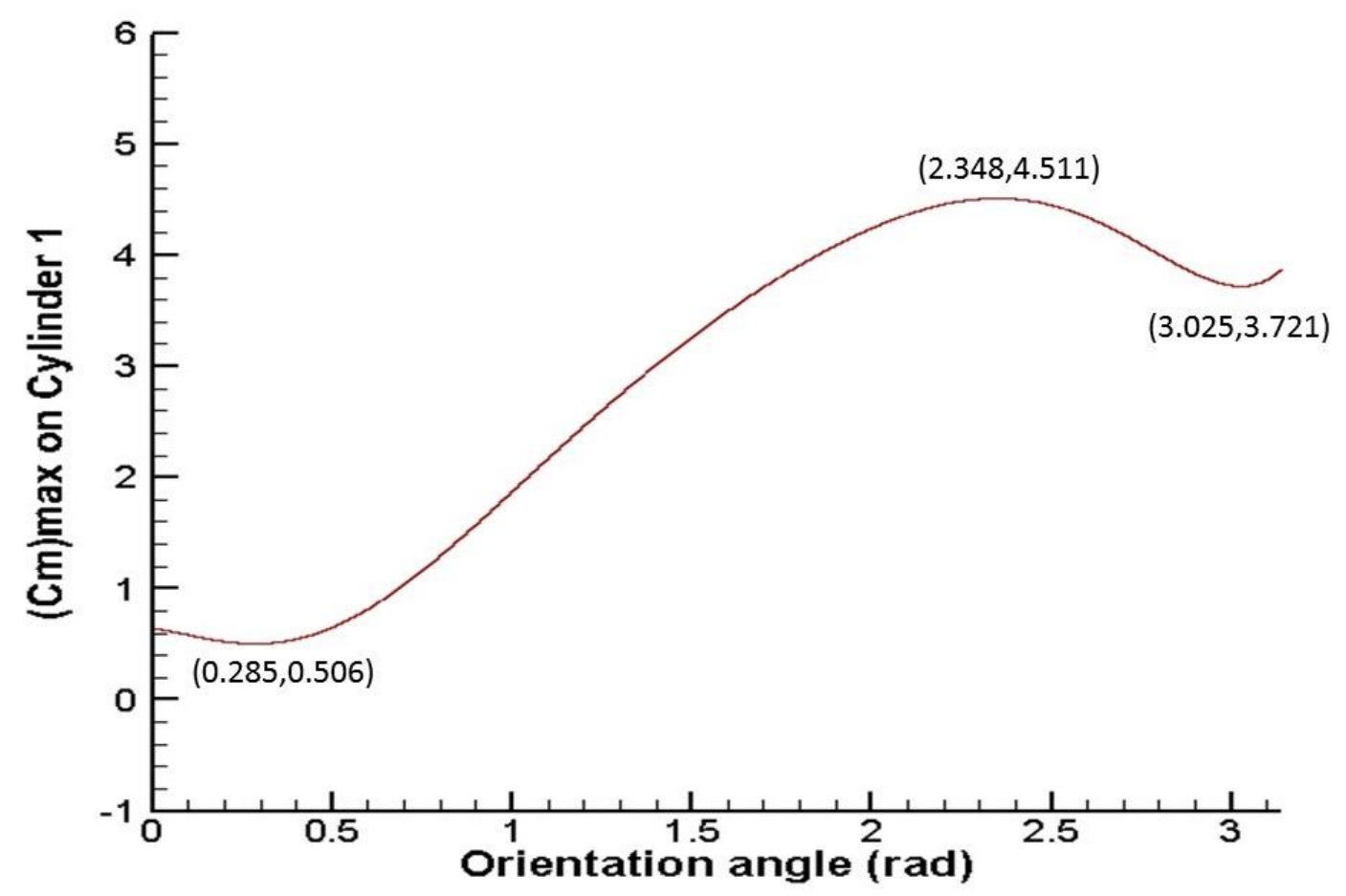

Figure 7.5 Variation of $7^{\text {th }}$ order $\left(C_{m}\right)_{\max }$ polynomial of cylinder 1 with $\theta$ 


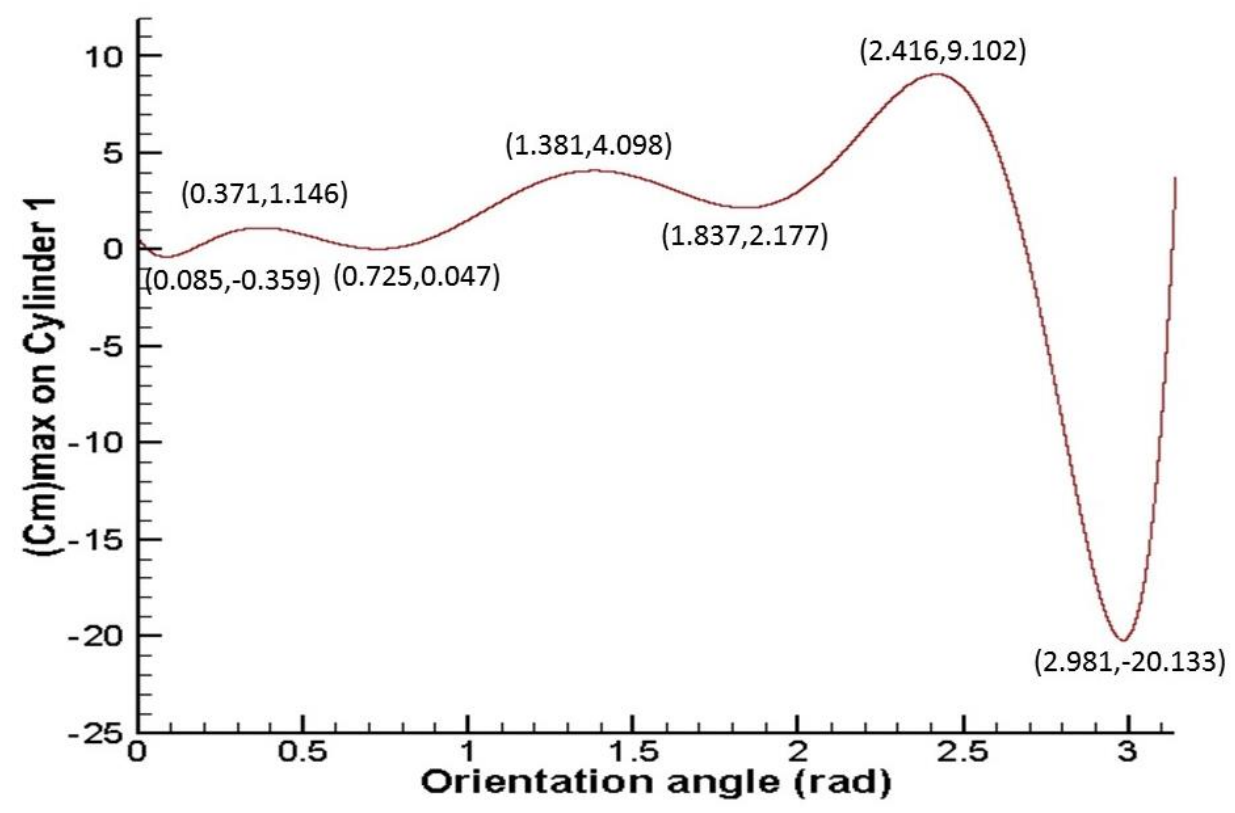

Figure 7.6 Variation of $8^{\text {th }}$ order $\left(C_{m}\right)_{\max }$ polynomial of cylinder 1 with $\theta$

Figure 7.4 and Figure 7.5 follow a similar trend of exhibiting a small dip in the region before $\theta=0.5 \mathrm{rad}$ and then increases monotonically to achieve maximum around $\theta=2.4 \mathrm{rad}$ and then falls to a minimum. But the Figure 7.6 shows a completely different pattern that has lot of ups and downs before reaching a relatively higher maximum and then falls to a very lower value being on the negative side, which is not the reality. But a careful examination of all the three curves shows a safe zone roughly in the range $\theta=0$ to $2.2 \mathrm{rad}$, where the value of $\left(C_{m}\right)_{\max }$ on cylinder 1 is below 5.0.

\subsubsection{Polynomial for $\left(C_{m}\right)_{\min }$ on Cylinder 1}

The data sets obtained for $\left(C_{m}\right)_{\min }$ on cylinder 1 for different values of orientation angles are given in Table 7.3. Aforementioned procedure to obtain the polynomials of different orders are same, but the last two data sets are found by re-running the test cases at the maximum coefficient value, i.e. lowest magnitude values and not at the minimum coefficient values, which are used for the other two coefficients $\left(C_{F}\right)_{\max }$, and $\left(C_{m}\right)_{\max }$. The last two stationary orientation angles in 
the table $(0.642$ and $0.601 \mathrm{rad})$ are the maximum values of the polynomials of sixth and seventh orders respectively.

Table 7.3 Variation of $\left(C_{m}\right)_{\min }$ with $\theta$ for cylinder 1

\begin{tabular}{|c|c|c|c|c|c|c|c|c|c|}
\hline $\begin{array}{c}\text { Orientatio } \\
\mathbf{n} \text { angle } \boldsymbol{\theta} \\
(\mathbf{r a d})\end{array}$ & 0 & 0.5236 & 1.0472 & 1.5708 & 2.0944 & 2.6180 & 3.1416 & 0.642 & 0.601 \\
\hline$\left(\boldsymbol{C}_{\boldsymbol{m}}\right)_{\min }$ & -2.4604 & -2.2259 & -3.4926 & -7.3155 & -10.1254 & -12.2445 & -11.0728 & -2.2052 & -2.2116 \\
\hline
\end{tabular}

The polynomials of three different orders for $\left(C_{m}\right)_{\min }$ on cylinder 1 are given in the Eq. (7.8), (7.9), and (7.10) below:

$$
\begin{aligned}
\left(C_{m}\right)_{\min }= & 1.0337 \theta^{6}-9.9221 \theta^{5}+36.0694 \theta^{4}-59.9745 \theta^{3}+41.1538 \theta^{2} \\
- & 9.1345 \theta-2.4604 \\
\left(C_{m}\right)_{\min }= & 0.4630 \theta^{7}-4.0582 \theta^{6}+12.2974 \theta^{5}-12.7723 \theta^{4}-3.4317 \theta^{3} \\
& +8.9923 \theta^{2}-2.2604 \theta-2.4604 \\
\left(C_{m}\right)_{\min }=- & 2.3242 \theta^{8}+27.5126 \theta^{7}-131.9905 \theta^{6}+329.1829 \theta^{5} \\
& -454.0806 \theta^{4}+340.2642 \theta^{3}-129.2117 \theta^{2}+20.0422 \theta \\
& -2.5981
\end{aligned}
$$

Figure 7.7, Figure 7.8, and Figure 7.9 represent the polynomial curves of $\left(C_{m}\right)_{\min }$ on cylinder 1 of orders six, seven, and eight respectively. All the three figures follow a very much similar trend, except for a steep rise to a local maximum in the Figure 7.9 in its final stages. As opposed to the curves of $\left(C_{F}\right)_{\max }$ and $\left(C_{m}\right)_{\max }$ that increase in the most part of the curves with the orientation angle, the $\left(C_{m}\right)_{\min }$ curve, after initial ups and downs, decrease with the orientation angle for the major portion of the curve. Again a safe zone can be sensed roughly in the region between $\theta=0$ and $1.4 \mathrm{rad}$ where the magnitude of the minimum moment coefficient is below 6.0. 


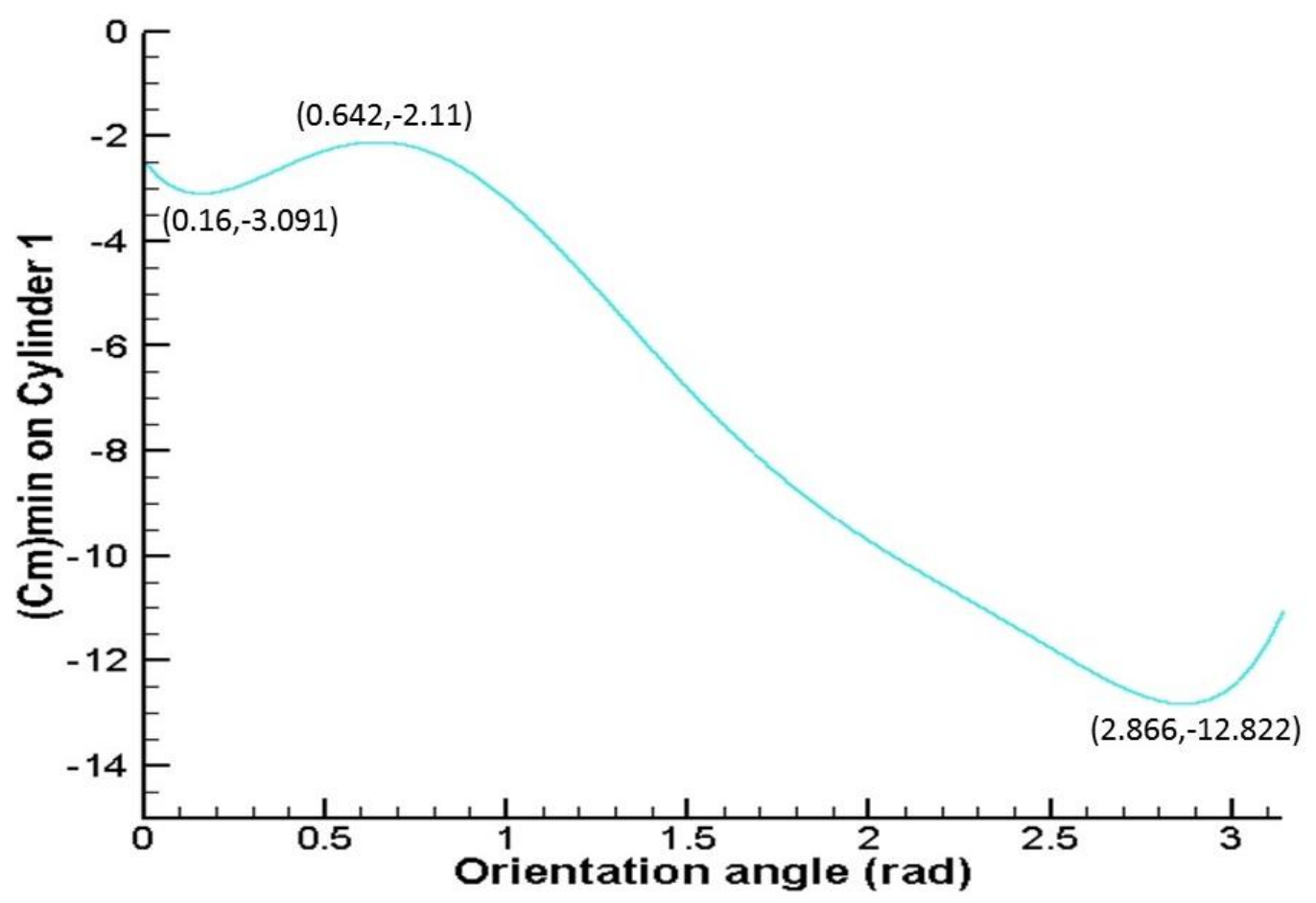

Figure 7.7 Variation of $6^{\text {th }}$ order $\left(C_{m}\right)_{\text {min }}$ polynomial of cylinder 1 with $\theta$

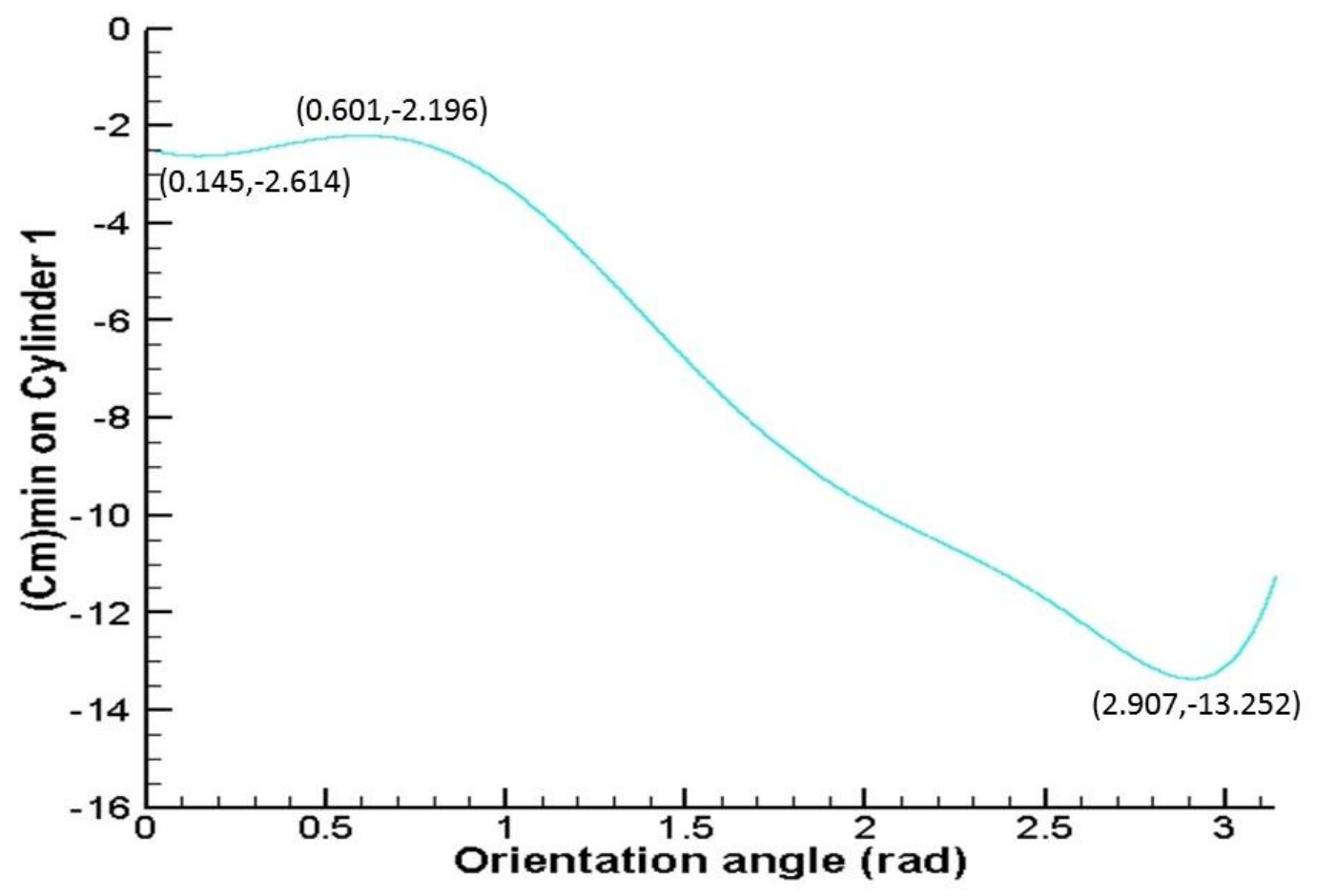

Figure 7.8 Variation of $7^{\text {th }}$ order $\left(C_{m}\right)_{\text {min }}$ polynomial of cylinder 1 with $\theta$ 


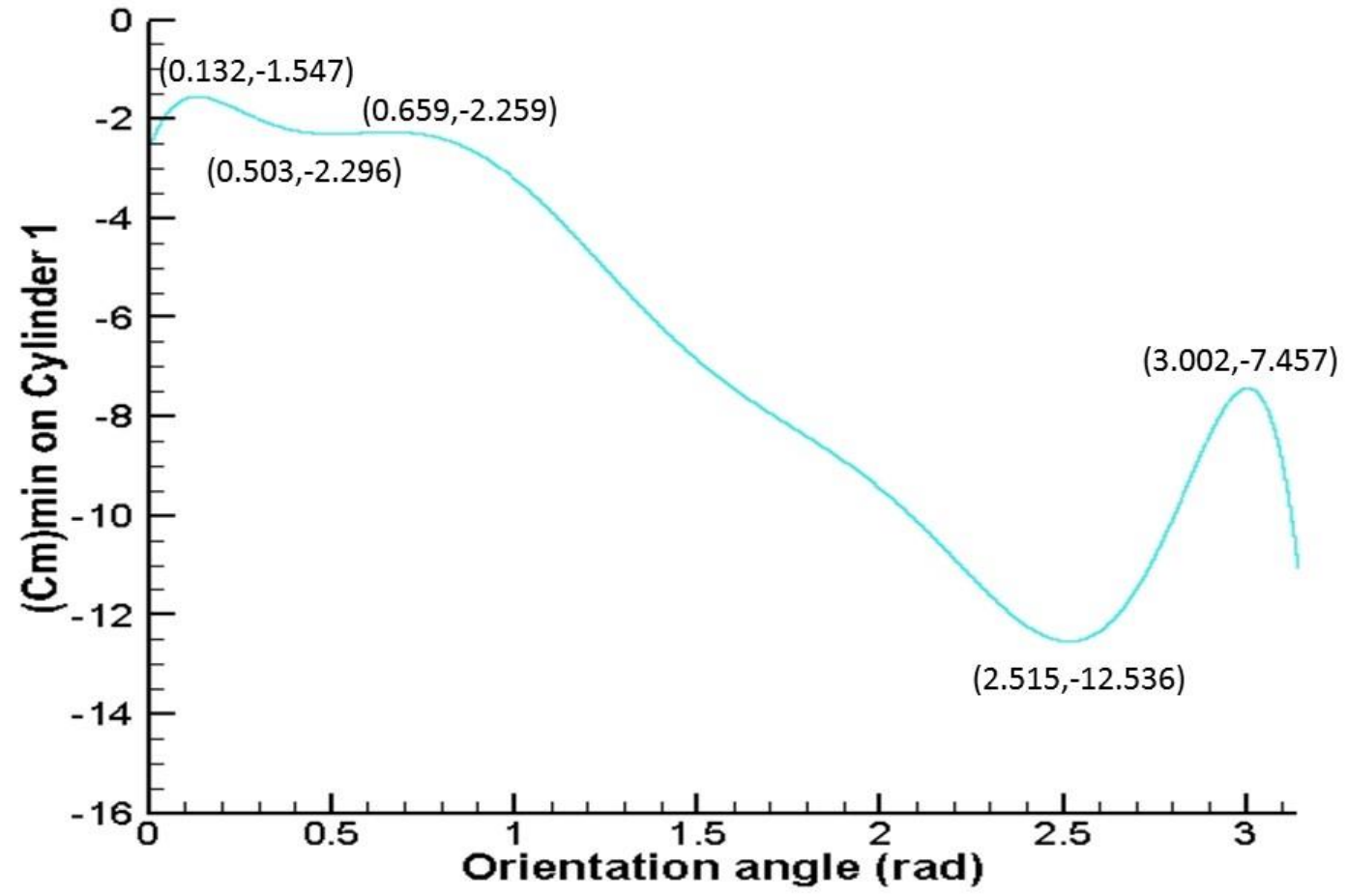

Figure 7.9 Variation of $8^{\text {th }}$ order $\left(C_{m}\right)_{\min }$ polynomial of cylinder 1 with $\theta$

\subsubsection{Safe Zone for Cylinder 1}

With the individual safe zone detected for the three parameters, the complete safe zone for cylinder 1 can be identified. The safe zones of the three coefficients and the overall safe zone for cylinder 1 are mentioned in the Table 7.4 .

Table 7.4 Safe zone for cylinder 1

\begin{tabular}{|c|c|c|}
\hline Coefficient & $\begin{array}{c}\text { Individual Safe Zone } \\
\text { (rad) }\end{array}$ & Safe Zone for Cylinder 1 \\
\hline$\left(C_{F}\right)_{\max }$ & 0 to 1.2 & 0 to $1.2 \mathrm{rad}$ \\
\hline$\left(C_{m}\right)_{\max }$ & 0 to 2.2 & (or) \\
\hline$\left(C_{m}\right)_{\min }$ & 0 to 1.4 & 0 to 69 degrees \\
\hline
\end{tabular}

The overall safe zone for cylinder 1 can be deduced from the three individual safe zones by taking the common range of orientation angles among them and it has been identified to be between 
$\theta=0$ and $1.2 \mathrm{rad}$, which is approximately equal to $0^{\circ}$ to $69^{\circ}$. In other words, for a given 7-cylinder model, when a tornado approaches the model with an orientation angle between 0 and 69 degrees, the wind-loadings on cylinder 1 would be considerably lower compared to the rest of the orientation angles and Figure 7.10 shows the schematic of the safe zone of cylinder 1.

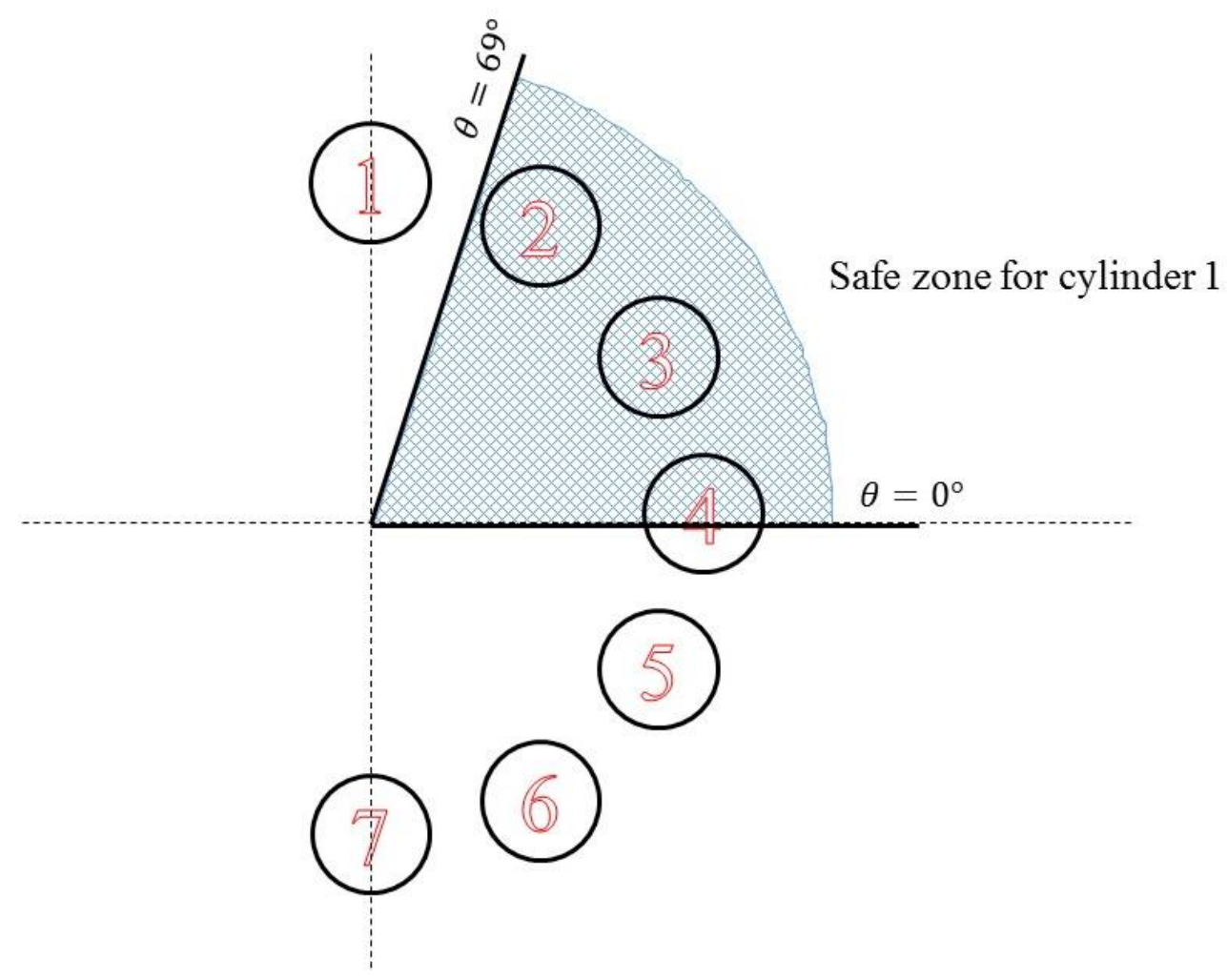

Figure 7.10 Safe zone for cylinder 1

\subsection{Optimization of the Overall 7 - Cylinder Model}

\subsubsection{Polynomial for Overall $\left(C_{F}\right)_{\max }$}

Table 7.4 The variation of the overall values of $\left(C_{F}\right)_{\max }$ with the orientation angles are given in Table 7.5. It is noted that these maximum coefficient values listed in the table are indeed the overall maximum coefficients of the whole seven cylinder arrangement irrespective of the cylinder 
on which the maximum coefficient occurs. In other words, for a specific orientation angle, this is the maximum coefficient experienced by the complete 7-cylinder model.

Table 7.5 Variation of overall $\left(C_{F}\right)_{\max }$ with $\theta$

\begin{tabular}{|c|c|c|c|c|c|c|c|c|c|}
\hline $\begin{array}{c}\text { Orientation } \\
\text { angle } \boldsymbol{\theta} \\
(\text { rad) }\end{array}$ & 0 & 0.5236 & 1.0472 & 1.5708 & 2.0944 & 2.6180 & 3.1416 & 0.1942 & 0.661 \\
\hline$\left(\boldsymbol{C}_{\boldsymbol{F}}\right)_{\max }$ & 61.0870 & 60.3844 & 61.0558 & 61.5281 & 81.7222 & 102.6730 & 88.8559 & 65.8338 & 64.2261 \\
\hline
\end{tabular}

The polynomials for overall $\left(C_{F}\right)_{\max }$ of sixth, seventh, and eighth orders are given below in the Eq. (7.11), (7.12), and (7.13)respectively:

$$
\begin{aligned}
\left(C_{F}\right)_{\max } & =5.5745 \theta^{6}-56.5678 \theta^{5}+208.7651 \theta^{4}-341.9908 \theta^{3} \\
& +247.8789 \theta^{2}-63.3076 \theta+61.0870 \\
\left(C_{F}\right)_{\max } & =9.7246 \theta^{7}-101.3528 \theta^{6}+409.9919 \theta^{5}-817.2557 \theta^{4} \\
& +845.0195 \theta^{3}-427.2189 \theta^{2}+80.9704 \theta+61.0869 \\
\left(C_{F}\right)_{\max }= & -42.7305 \theta^{8}+487.8702 \theta^{7}-2242.6986 \theta^{6}+5316.5429 \theta^{5} \\
& -6908.6189 \theta^{4}+4824.3782 \theta^{3}-1637.2785 \theta^{2}+204.0945 \theta \\
& +61.0852
\end{aligned}
$$

Figure 7.11, Figure 7.12, and Figure 7.13 represent the graphical form of the above three equations. Again all the three figures follow an identical pattern, except for an enormous higher maximum value seen in Figure 7.13 in the closing stage of the curve. Furthermore, it shows a steep decrease achieving a minimum value in its last phase, which is not visible in the other two figures. It is noted that the difference in characteristics can be seen only in the concluding stages of the curves and thereby a safe zone exists approximately in the region between $\theta=0$ and 1.7 rad where the coefficients shuttle between the values 50.0 and 65.0. 


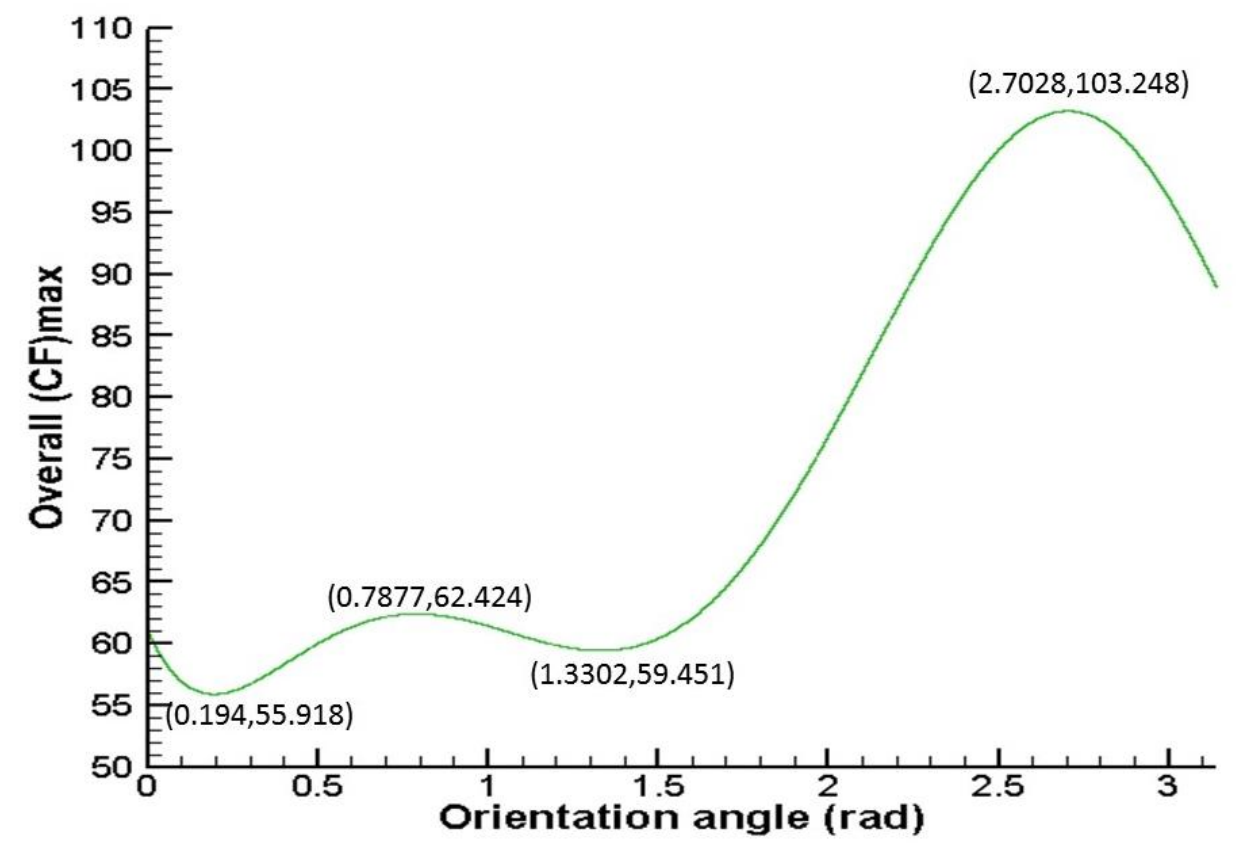

Figure 7.11 Variation of $6^{\text {th }}$ order overall $\left(C_{F}\right)_{\max }$ polynomial with $\theta$

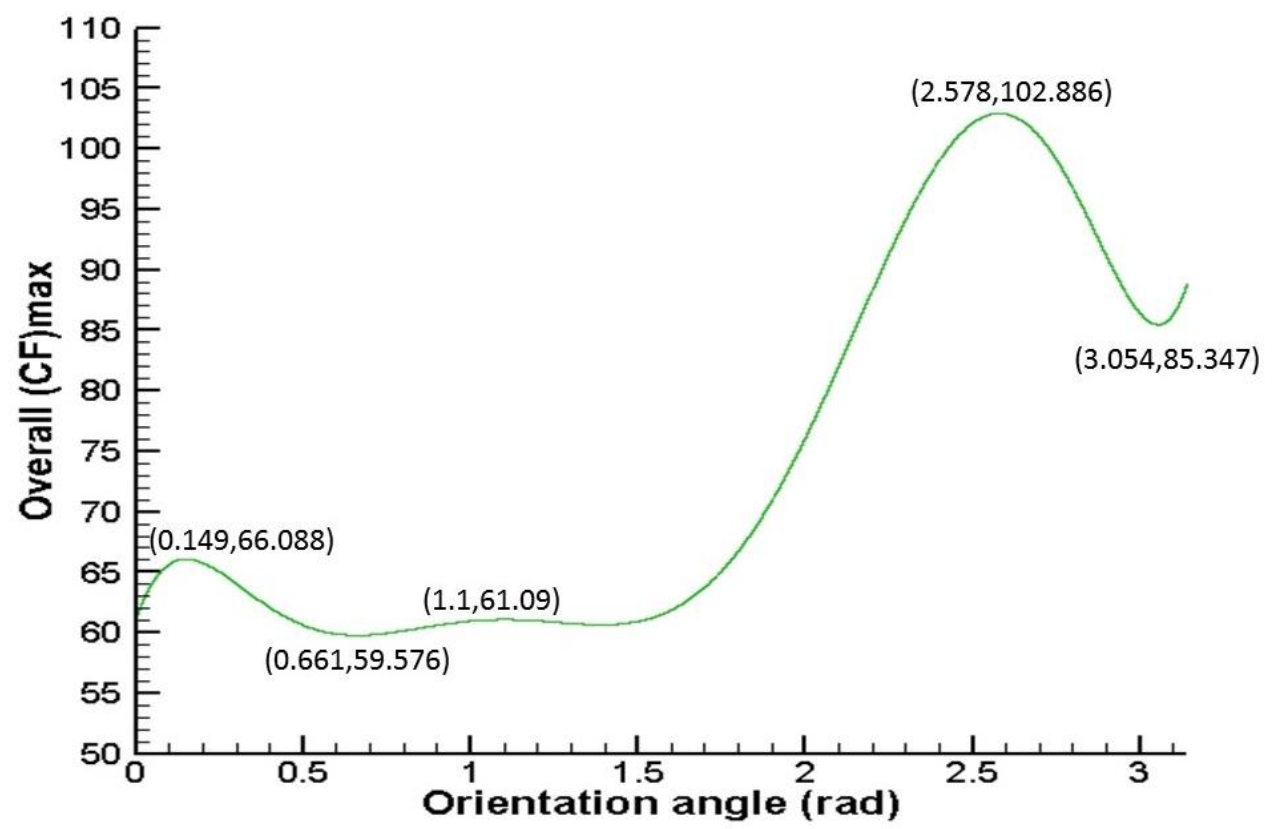

Figure 7.12 Variation of $7^{\text {th }}$ order overall $\left(C_{F}\right)_{\max }$ polynomial with $\theta$ 


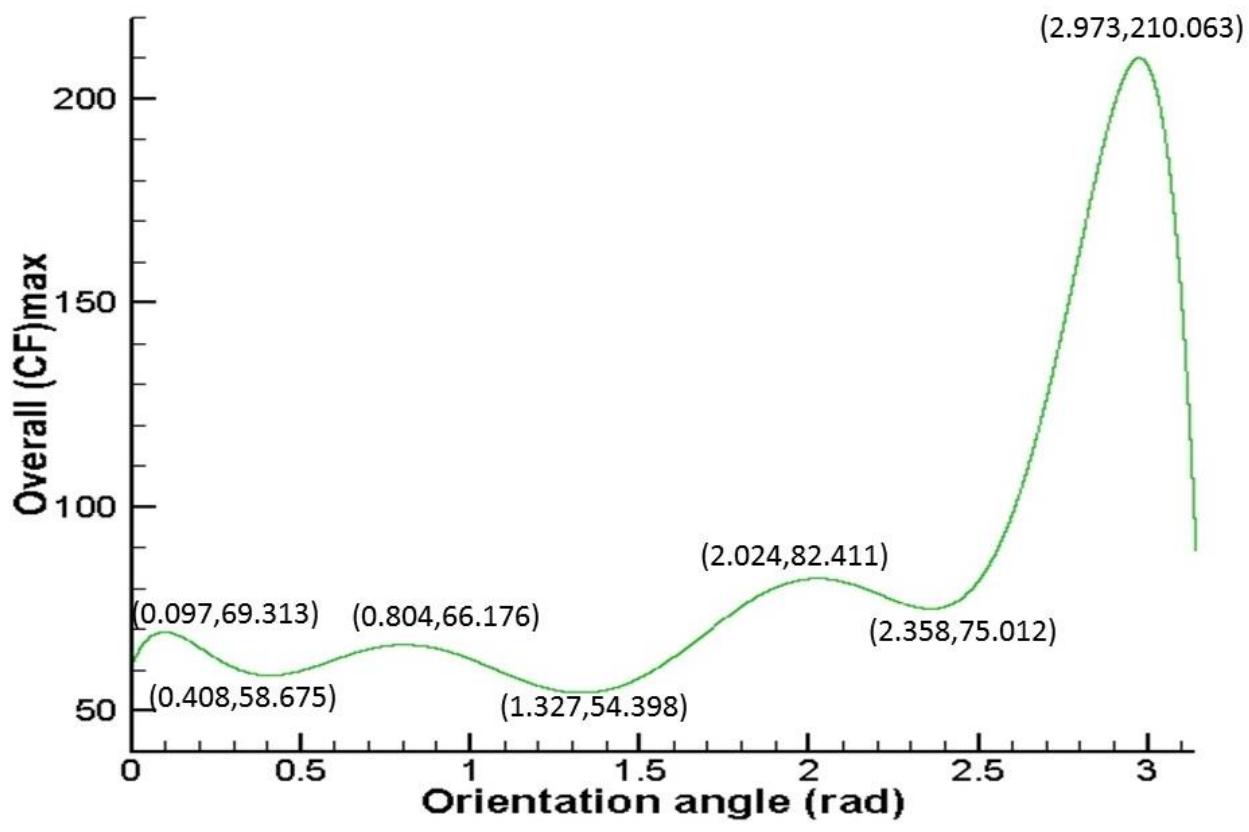

Figure 7.13 Variation of $8^{\text {th }}$ order overall $\left(C_{F}\right)_{\max }$ polynomial with $\theta$

\subsubsection{Polynomial for Overall $\left(C_{m}\right)_{\max }$}

The variation of overall $\left(C_{m}\right)_{\max }$ values with the orientation angles are given in Table 7.6 and the Eq. (7.14), (7.15), and (7.16) represent the polynomials of orders 6,7 , and 8 respectively. It is important to remember that the values listed in it are maximum values at their respective orientation angles regardless of the cylinders on which those occurred.

Table 7.6 Variation of overall $\left(C_{m}\right)_{\max }$ with $\theta$

\begin{tabular}{|c|c|c|c|c|c|c|c|c|c|}
\hline $\begin{array}{c}\text { Orientation } \\
\text { angle } \boldsymbol{\theta} \\
(\mathbf{r a d})\end{array}$ & 0 & 0.5236 & 1.0472 & 1.5708 & 2.0944 & 2.6180 & 3.1416 & 2.940 & 0.211 \\
\hline$\left(\boldsymbol{C}_{\boldsymbol{m}}\right)_{\max }$ & 5.0154 & 3.8797 & 5.8935 & 8.0970 & 7.7926 & 4.3316 & 3.9407 & 4.4999 & 4.5635 \\
\hline
\end{tabular}

$$
\begin{aligned}
\left(C_{m}\right)_{\max }= & 0.2049 \theta^{6}-1.2309 \theta^{5}+2.9389 \theta^{4}-6.8803 \theta^{3}+13.0844 \theta^{2} \\
& -7.4711 \theta+5.0154
\end{aligned}
$$




$$
\begin{aligned}
\left(C_{m}\right)_{\max } & =-1.6537 \theta^{7}+18.3882 \theta^{6}-80.5705 \theta^{5}+177.4164 \theta^{4} \\
- & 208.7345 \theta^{3}+127.8865 \theta^{2}-32.0060 \theta+5.0154 \\
\left(C_{m}\right)_{\max } & =-0.8271 \theta^{8}+9.8724 \theta^{7}-48.0315 \theta^{6}+123.3602 \theta^{5} \\
- & 180.1024 \theta^{4}+145.5009 \theta^{3}-53.1957 \theta^{2}+4.0713 \theta \\
+ & 5.0154
\end{aligned}
$$

Figure 7.14, Figure 7.15, and Figure 7.16 represent the graphical curves of the equations (7.14), (7.15), and (7.16) respectively. A careful examination of all the three figures reveal two safe zones: a) $\theta=0.2$ to $1.2 \mathrm{rad}, \mathrm{b}) \theta=2.4$ to $3.14 \mathrm{rad}$. In both the safe zones, the values of the overall $\left(C_{m}\right)_{\max }$ range between 2.0 and 6.0. In the non-safe zone, the coefficient increases with orientation angle and achieves the maximum which is around 8.0 in all the three figures.

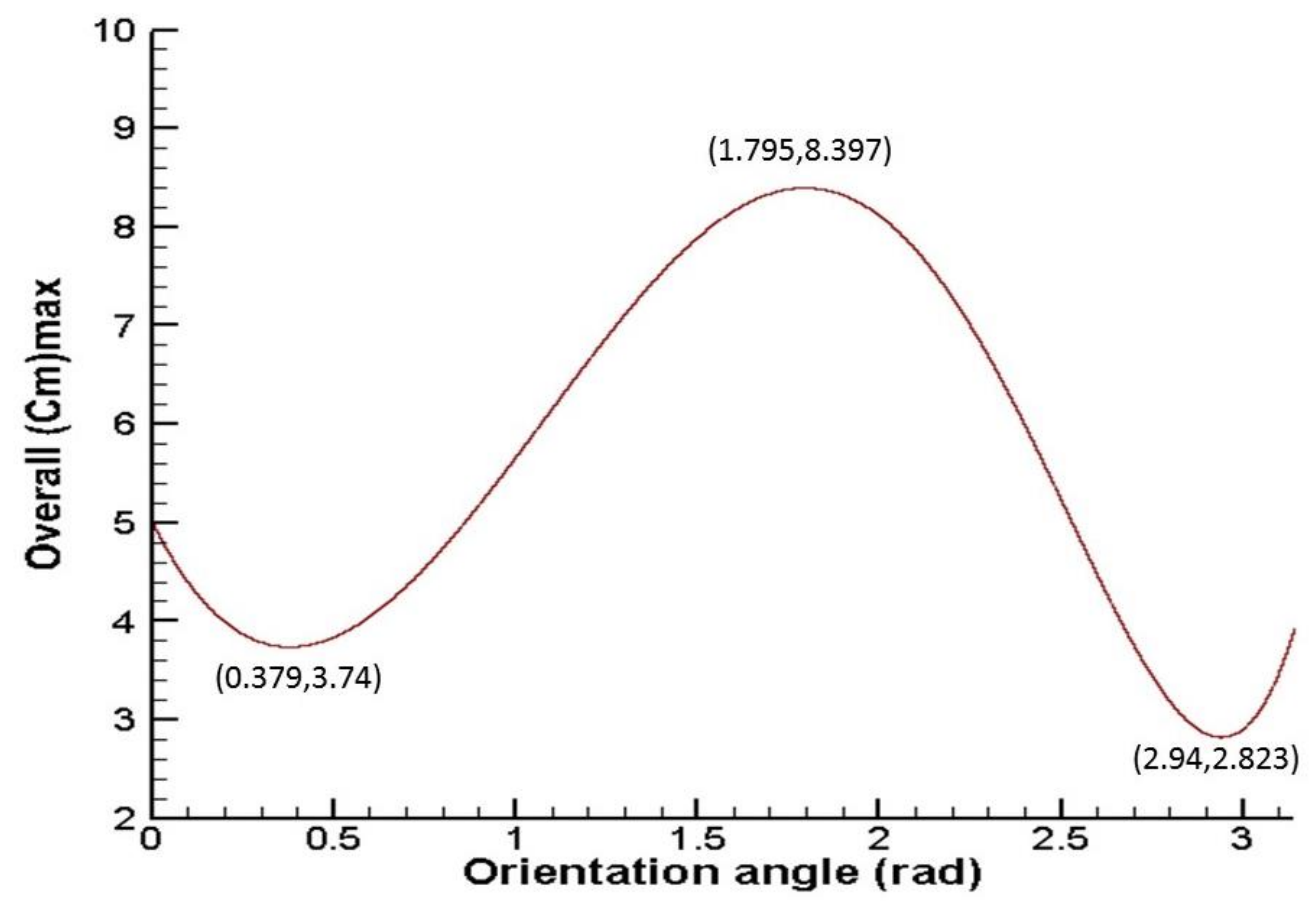

Figure 7.14 Variation of $6^{\text {th }}$ order overall $\left(C_{m}\right)_{\max }$ polynomial with $\theta$ 


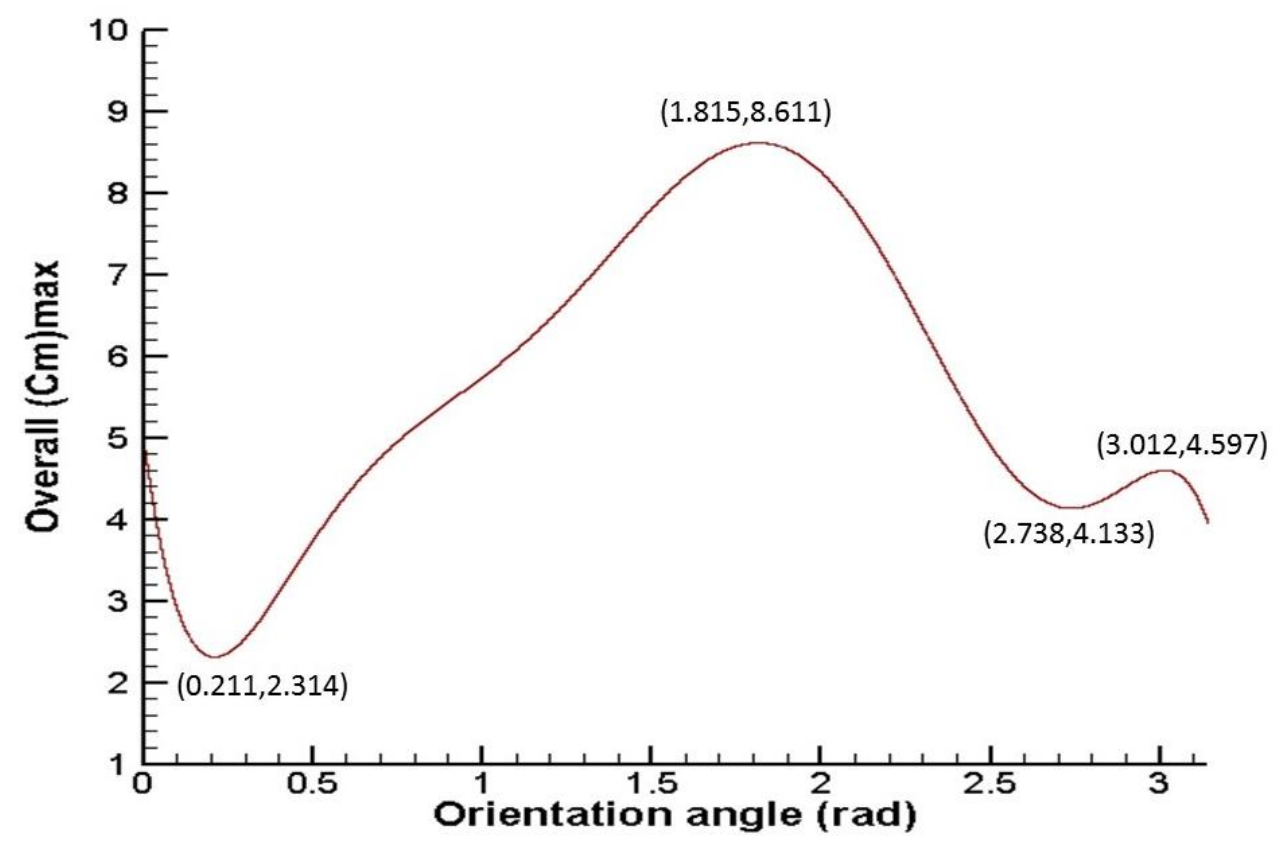

Figure 7.15 Variation of $7^{\text {th }}$ order overall $\left(C_{m}\right)_{\max }$ polynomial with $\theta$

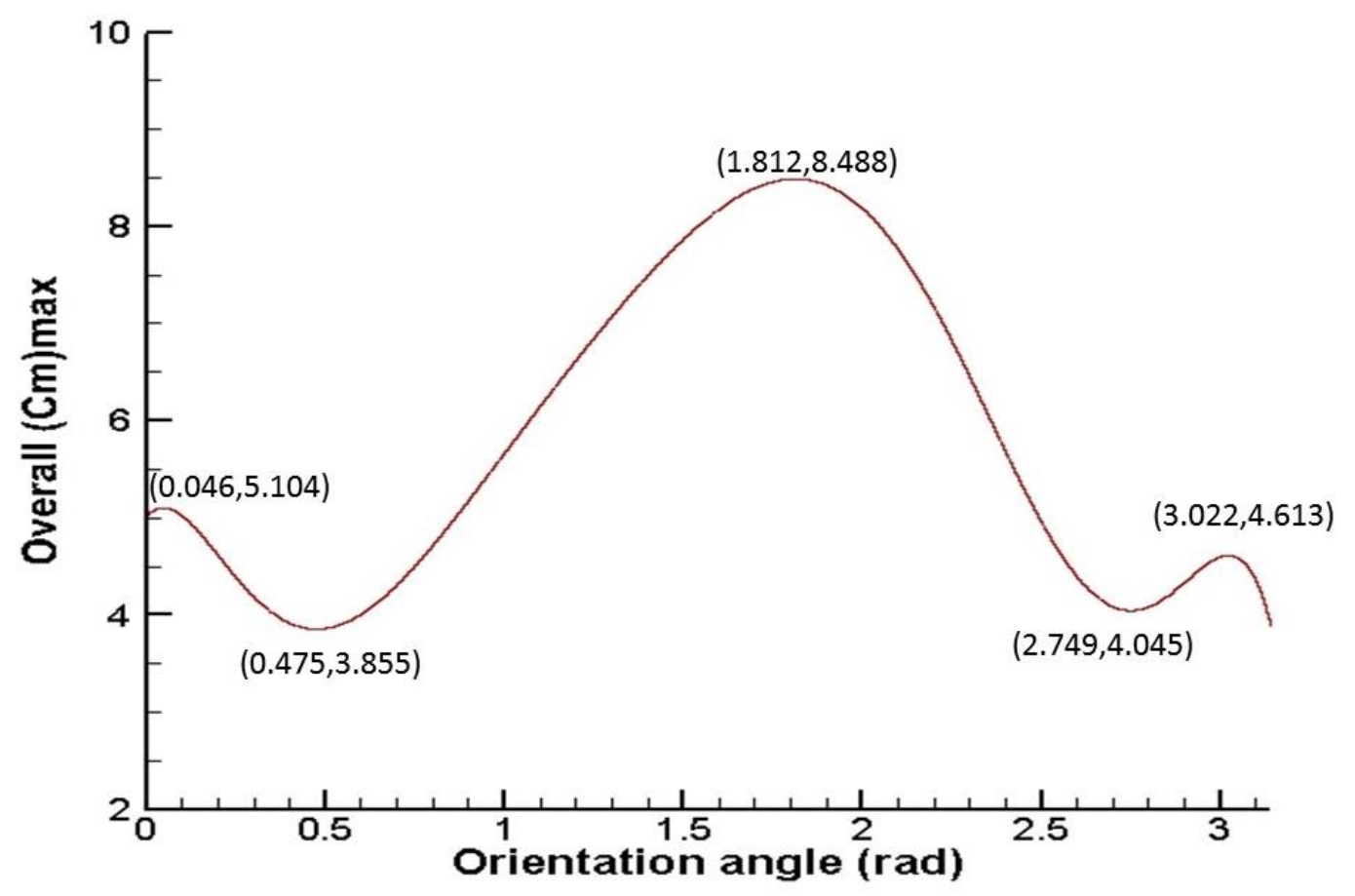

Figure 7.16 Variation of $8^{\text {th }}$ order overall $\left(C_{m}\right)_{\max }$ polynomial with $\theta$ 


\subsubsection{Polynomial for overall $\left(C_{m}\right)_{\min }$}

Table 7.7 shows the variation of overall $\left(C_{m}\right)_{\min }$ values with the orientation angle and the corresponding polynomials of orders 6, 7, and 8 are expressed in the Eq. (7.17), (7.18), and (7.19) respectively.

Table 7.7 Variation of overall $\left(C_{m}\right)_{\min }$ with $\theta$

\begin{tabular}{|c|c|c|c|c|c|c|c|c|c|}
\hline $\begin{array}{c}\text { Orientatio } \\
\text { n angle } \boldsymbol{\theta} \\
(\mathbf{r a d})\end{array}$ & 0 & 0.5236 & 1.0472 & 1.5708 & 2.0944 & 2.6180 & 3.1416 & 0.764 & 0.166 \\
\hline$\left(\boldsymbol{C}_{\boldsymbol{m}}\right)_{\min }$ & -8.2213 & -8.0780 & -8.3162 & -11.9023 & -13.5647 & -12.2445 & -11.0728 & -9.3637 & -11.2004 \\
\hline
\end{tabular}

$$
\begin{aligned}
\left(C_{m}\right)_{\min }= & 0.8407 \theta^{6}-9.2395 \theta^{5}+37.9628 \theta^{4}-70.2431 \theta^{3}+54.7234 \theta^{2} \\
- & 13.9100 \theta-8.2213 \\
\left(C_{m}\right)_{\min }= & 7.8563 \theta^{7}-84.5315 \theta^{6}+359.7358 \theta^{5}-767.3547 \theta^{4} \\
+ & 856.0318 \theta^{3}+469.8326 \theta^{2}+97.8700 \theta-8.2213 \\
\left(C_{m}\right)_{\min }= & 15.4723 \theta^{8}-174.5184 \theta^{7}+791.4442 \theta^{6}-1852.3608 \theta^{5} \\
+ & 2389.8387 \theta^{4}-1680.3276 \theta^{3}+589.1016 \theta^{2}-79.0549 \theta \\
- & 8.2214
\end{aligned}
$$

The graphical representation of the three polynomials are depicted in the Figure 7.17, Figure 7.18, and Figure 7.19 respectively. As far as the overall $\left(C_{m}\right)_{\min }$ is concerned, the safe zone is one in which the values of the coefficients are higher, i.e. the coefficients have lower magnitudes. All the three figures reveal a safe zone between $\theta=0.5$ and $1.2 \mathrm{rad}$, where the value of the coefficient ranges below -12.0 . Once the safe is crossed, the values tend to rise very steeply in the negative region that is evident in Figure 7.19. 


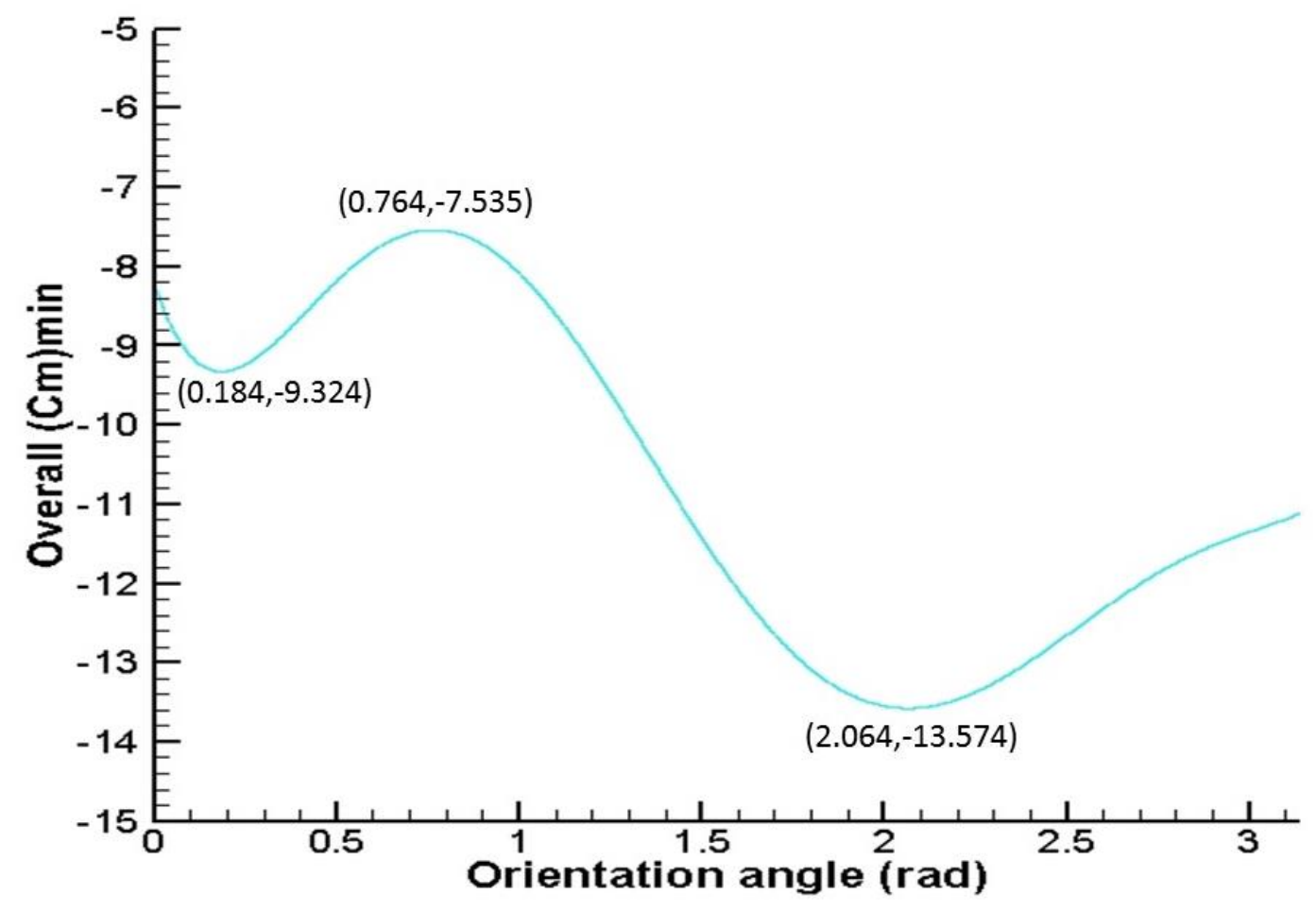

Figure 7.17 Variation of $6^{\text {th }}$ order overall $\left(C_{m}\right)_{\min }$ polynomial with $\theta$

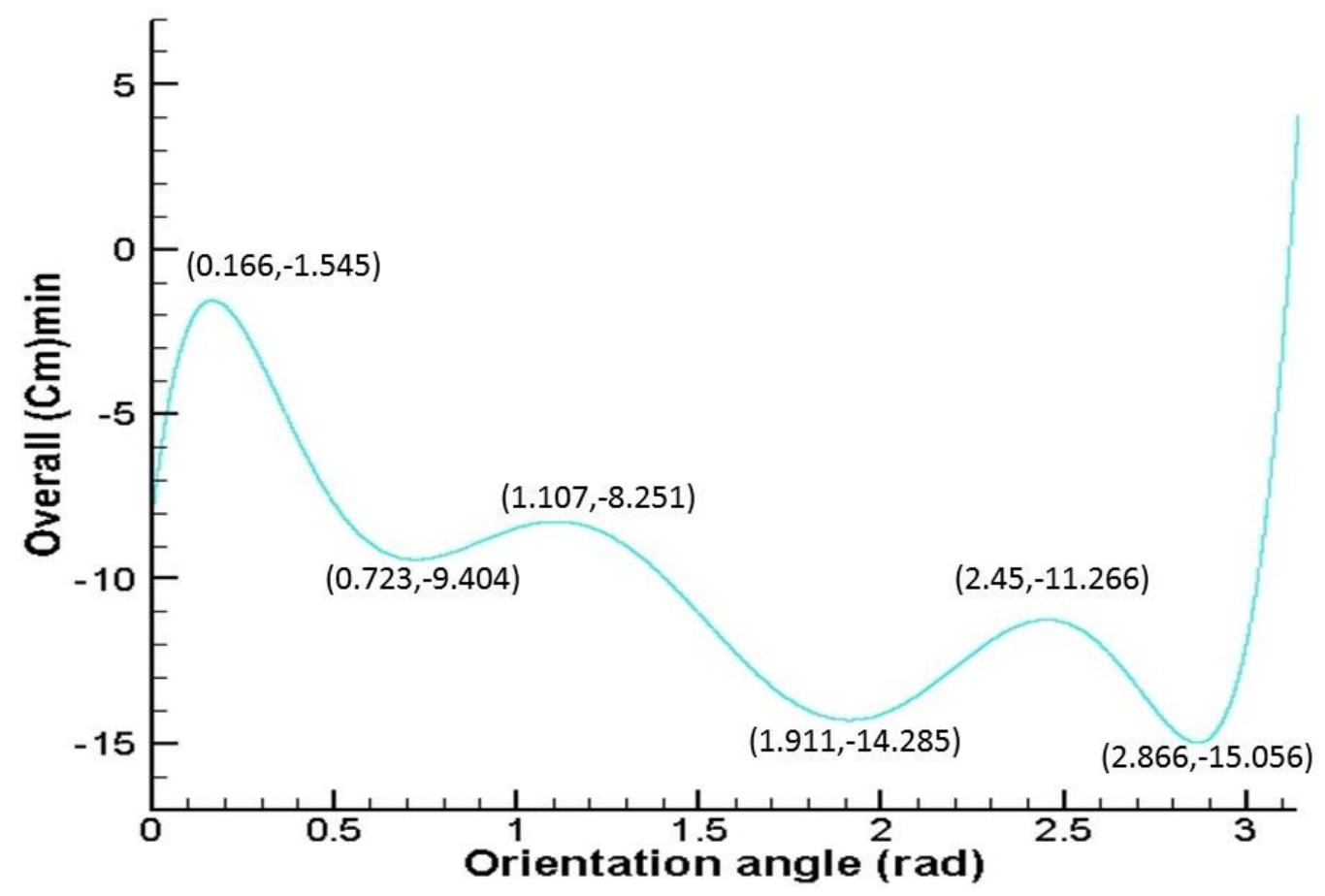

Figure 7.18 Variation of $7^{\text {th }}$ order overall $\left(C_{m}\right)_{\min }$ polynomial with $\theta$ 


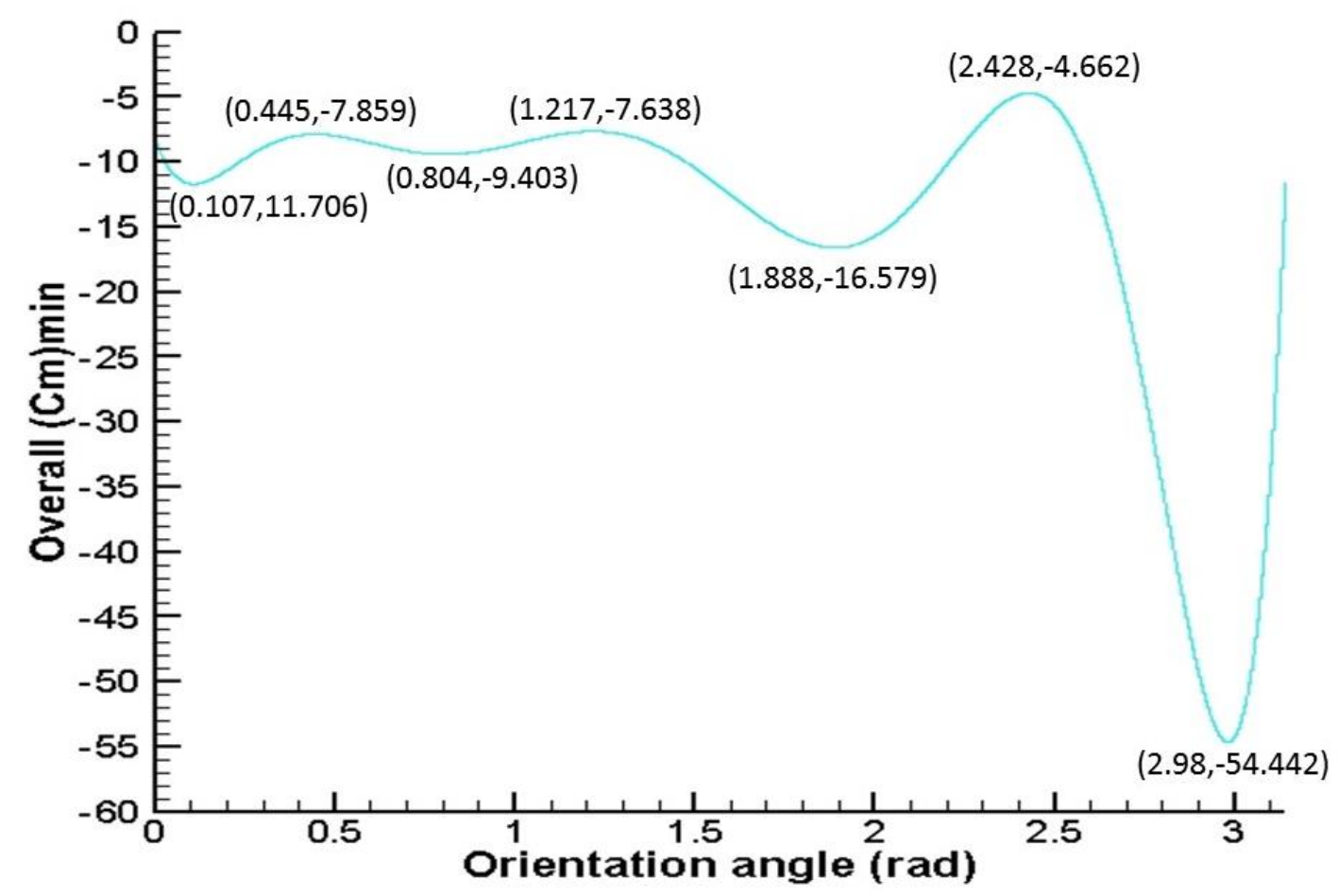

Figure 7.19 Variation of $8^{\text {th }}$ order overall $\left(C_{m}\right)_{\min }$ polynomial with $\theta$

\subsubsection{Overall Safe Zone for the 7 - Cylinder Model}

Using the polynomials of orders 6,7 , and 8 , safe zones are identified with respect to each defined wind-loading coefficients. In order to find a compromising safe zone with respect to the whole seven building model, the individual safe zones have been looked at carefully that are presented in Table 7.8. After a serious examination of the individual safe zones, it is found that the safe zone is in the orientation angle range, $\theta=0.5$ to $1.2 \mathrm{rad}$ or 29 to 69 degrees approximately. In the safe zone, all the three coefficients exhibit immensely lower magnitudes relative to the magnitudes experienced in the non-safe zone range. It leads to a great result that, in order to have lesser wind-loading coefficients, for a given tornado direction, the orientation angle should be maintained between 29 and 69 degrees, which is the overall safe zone for all the seven buildings in the model and the schematic of the overall safe zone is represented in Figure 7.20. 
Table 7.8 Overall safe zone for the complete 7-cylinder model

\begin{tabular}{|c|c|c|}
\hline Coefficient & $\begin{array}{c}\text { Individual Safe Zone } \\
\text { (rad) }\end{array}$ & $\begin{array}{c}\text { Safe Zone for Complete } \\
\text { Model }\end{array}$ \\
\hline$\left(C_{F}\right)_{\max }$ & 0 to 1.7 & 0.5 to $1.2 \mathrm{rad}$ \\
\hline$\left(C_{m}\right)_{\max }$ & $\begin{array}{c}0.2 \text { to } 1.2 \\
\&\end{array}$ & (or) \\
& 2.4 to 3.14 & 29 to 69 degrees \\
\hline$\left(C_{m}\right)_{\min }$ & 0.5 to 1.2 & \\
\hline
\end{tabular}

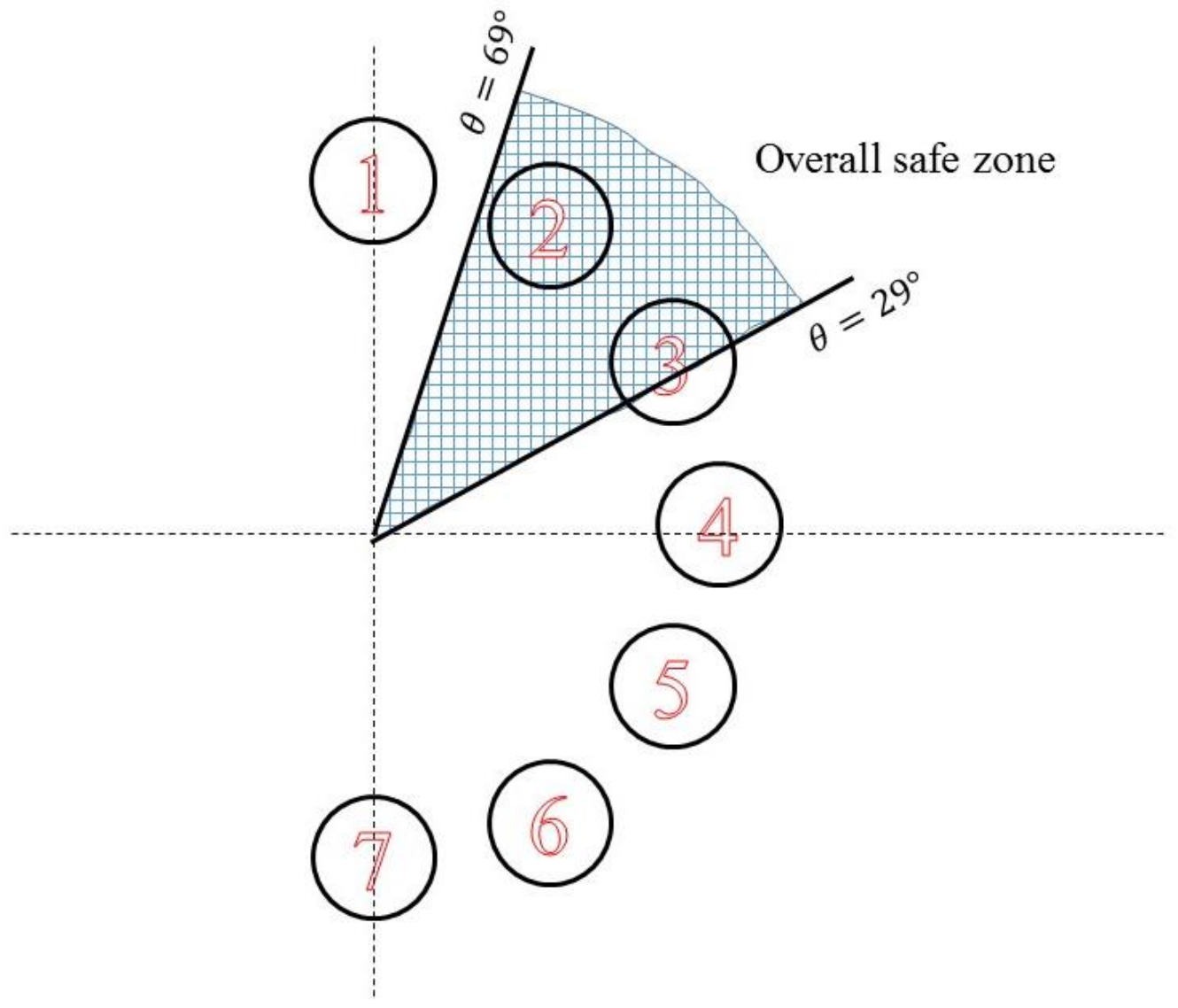

Figure 7.20 Overall safe zone for the complete 7-cylinder model 


\subsection{Comparison of the Overall and Cylinder 1 Safe Zones}

The safe zone for cylinder 1 and overall safe zone for the complete 7-cylinder model are found to be $0^{\circ}$ to $69^{\circ}$ and $29^{\circ}$ to $69^{\circ}$ respectively. As already mentioned, the cylinder 1 experiences the maximum force and moment coefficients for the orientation angle between $90^{\circ}$ and $180^{\circ}$. Therefore, it can be easily understood that the safe zone for cylinder 1 exists to be between $0^{\circ}$ and $69^{\circ}$. The safe range for the overall model is lesser than the safe range for the cylinder 1 , for the other cylinders in the model experience the higher wind-loadings in the missing range $0^{\circ}$ to $29^{\circ}$. Despite the fact that the cylinder 1 has found be the most affected cylinder in the model, cylinder 1 alone should not be considered to minimize the wind-loadings in the entire model. 


\section{Chapter 8 - Conclusions}

\subsection{Concluding Remarks}

In this study, the conventional Rankine Combined Vortex Model (RCVM) has been modified in order to ease the implementation of boundary conditions required to set up the tornado-like flow in the computational domain. The modification has been performed using the concept of "relative motion", by which the translational component of the tornado, the root cause of the modification, has been detached from the tornado and attached to the seven cylinder building model and the modification has rendered the boundary conditions time-independent. The relative motion concept has been implemented via Immersed Boundary method (IBM) that modelled the buildings and its translation towards the tornado. Finally, the Lattice Boltzmann Method (LBM) has been integrated with the Immersed Boundary technique to form the complete numerical procedure, IB-LBM, to perform the whole simulation of investigating the tornado dynamics using the seven cylinder building model.

The focus of this study was mainly placed on the investigation of the effects of tornado dynamics on multi-bodies arranged in a semi-circular pattern at a specific angle of the tornado, and at a specific Reynolds number. The impact of tornado on the model has been analyzed using four wind-loading coefficients $C_{x}, C_{y}, C_{m}$, and $C_{F}$. The coefficients are determined and salient points on the curves are discussed to understand the behavior of tornadic wind. Due to the concurrent translation and rotation, the tornado, which was in line of fire against cylinder 4 initially, has moved more towards the cylinder 1 and has produced the greatest impact with very high wind-loading coefficients that was very evident from the Table 6.8. It also suggests that the buildings at the extreme ends are subjected to major destructions and needs to be have extra- 
ordinary wind resistance capability. Further by using an unconventional rule of thumb, the most affected or the unluckiest building has been identified to be the cylinder 1 .

The numerical results of this study gives a clear picture of the impact of the tornado on a group of buildings and the impact of buildings among themselves, called interference effects. Particularly, the effects of vortices produced by a cylinder on the wind-loadings of the other cylinders has been understood with the help of flow visualization pictures. The coefficient curves of the cylinders 4 to 6 , which have lesser wind-loadings, reveals the already mentioned dual nature, brutality and gentleness, of the tornado on buildings, which is one of the major subjects of this study.

A new parameter called "orientation angle" has been defined and the seven-cylinder building model has been tested at various orientation angles and the results of the test cases $A$ to $\mathrm{G}$ have been tabulated and examined. Further, the results in those tables have given a clear picture of the variation of the wind-loading coefficients on each cylinder in the model with the orientation angles.

A unique optimization procedure has been adopted to optimize the wind-loading coefficients experienced by the buildings in the model. The optimization method has greatly utilized "fitting a polynomial to a set of data" mathematical technique to find polynomials of three consecutive orders six, seven, and eight for the wind-loading coefficients. The same optimization procedure has been applied to both overall model and cylinder 1 wind-loading coefficients and identified safe zones for all the six wind-loading coefficients, three each for cylinder 1 and overall model.

On the basis of individual safe zones, an overall safe zone has been identified where the orientation angle ranges from 29 to 69 degrees. It is suggested that, in order for the buildings to 
experience lower wind-loadings, the orientation angle should be maintained between 29 and 69 degrees.

\subsection{Contributions}

- The Immersed Boundary-Lattice Boltzmann Method has been successfully applied to investigate the interaction between a tornado and a multi-structure arrangement.

- Building Orientation has been proved to be one of the main factors that affect the windloadings on the structures.

- Variation of wind-loadings with the building orientations has been studied.

- The cylinder at one of the extreme end, cylinder 1 , has been found to be the most affected building.

- An optimization procedure has been developed and employed to detect the safe orientation zones.

- The safe orientation zone for the most affected building has been detected to between $0^{\circ}$ and $69^{\circ}$.

- An overall safe orientation zone for the entire 7-buliding model has been identified to exist between $29^{\circ}$ and $69^{\circ}$.

\subsection{Future Prospects}

The subsequent work aims to employ this current numerical procedure to optimize the present seven cylinder building model in a tornadic wind at different Reynolds numbers and to study the effects of Reynolds number on the wind-loadings. The future works intend to define a new and more appropriate Reynolds number that accommodates both the rotational and translational components of the tornadic wind. The succeeding works can apply the current 2-D IB-LBM 
framework to analyze more complex geometries and more complicated flow situations. The ensuing works intend to employ different ideas to improve the accuracy of the present IB-LBM framework. In future, the current 2-D IB-LBM framework can be extended to 3-D and with the recent success in 3-D IB-LBM simulations ${ }^{[38]-[39]}$, the extension looks promising to getting developed into a 3-D numerical scheme to represent a more realistic vortex-structure interaction. 


\section{References}

[1] https://www.ncdc.noaa.gov/climate-information/extreme-events/us-tornado-climatology [Accessed on May 18, 2017].

[2] N.B. Ward, "The Exploration of Certain Features of Tornado Dynamics Using a Laboratory”. Journal of Atmospheric Science, 29, 1194-1204, 1972.

[3] C.A. Wan, and C.C. Chang, "Measurement of the Velocity Field in a Simulated Tornadolike Vortex Using a Three-Dimensional Probe”. Journal of Atmospheric Science, 29, 116127, 1972.

[4] C.R. Church, J.T. Snow, G.L. Baker, and E.M. Agee, "Characteristic of Tornado-like vortices as a Function of Swirl Ratio: A Laboratory Investigation". Journal of Atmospheric Science, 36, 1755-1776, 1979.

[5] R.P. Davies-Jones, "The Dependence of Core Radius on Swirl Ratio in a Tornado Simulator". Journal of Atmospheric Science, 30, 1427-1430, 1973.

[6] C.J. Diamond, and E.M. Wilkins, "Translation Effects on Simulated Tornadoes". Journal of Atmospheric Science, 41, 2574-2580, 1984.

[7] Y. Mitsuta, and N. Monji, "Development of a Laboratory Simulator for Small Scale Atmospheric Vortices”. Natural Disaster Science, 6, 43-54, 1984.

[8] M. Matsui, and Y. Tamura, "Influence of Swirl Ratio and Incident Flow Conditions on Generation of Tornado-like Vortex". European and African Conference on Wind Engineering, 5, 2009.

[9] W. Zhang, and P.P. Sarkar, "Near-ground Tornado-like Vortex Structure Resolved by Particle Image Velocimetry (PIV)”. Experiments in Fluids, 52, 479-493, 2012. 
[10] P.H. Tari, R. Gurka, and H. Hangan, "Experimental Investigation of Tornado-like Vortex Dynamics with Swirl Ratio: The Mean and Turbulent Flow Fields”. Journal of Wind Engineering and Industrial Aerodynamics, 98, 936-944, 2010.

[11] F.H. Harlow, and L.R. Stein, "Structural Analysis of Tornado-like Vortices". Journal of Atmospheric Science, 31, 2081-2098, 1974.

[12] R. Rotunno, "Numerical Simulation of a Tornado Vortex". Journal of Atmospheric Science, 34, 1942-1956, 1977.

[13] R. Rotunno, “A Study in Tornado-like Vortex Dynamics”. Journal of Atmospheric Science, 36, 140-156, 1979.

[14] D.S. Nolan, and B.F. Ferrell, “The Structure and Dynamics of Tornado-like Vortices". Journal of Atmospheric Science, 56, 2908-2936, 1999.

[15] D.C. Lewellen, and W.S. Lewellen, "Large Eddy Simulation of Tornado's Interaction with the Surface". Journal of Atmospheric Science, 54(5), 581-605, 1997.

[16] D.C. Lewellen, and W.S. Lewellen, "Near-surface Intensification of Tornado Vortices". Journal of Atmospheric Science, 64, 2176-2194, 2007.

[17] H. Hangan, and J.D. Kim, "Swirl Ratio Effects on Tornado Vortices in relation to the Fujita Scale”. Wind and Structures, 11(4), 291-302, 2008.

[18] T. Ishihara, S. Oh, Y. Tokuyama, "Numerical Study on Flow Fields of Tornado-like Vortices Using the LES Turbulence Model". Journal of Wind Engineering Applications and Industrial Aerodynamics, 99(4), 239-248, 2011.

[19] D. Natarajan, and H. Hangan, "Large Eddy Simulation of Translation and Surface Roughness Effects on Tornado-like Vortices". Journal of Wind Engineering Applications and Industrial Aerodynamics, 104-106, 577-584, 2012. 
[20] S. Chen, G.D. Doolen, "Lattice Boltzmann Method for Fluid Flows". Annual Revision Fluid Mechanics, 30 (1), 329-364, 1998.

[21] S. Succi, R. Benzi, F. Higuera, "The Lattice Boltzmann Equation: A New Tool for Computational Fluid Dynamics”. Physica D Nonlinear Phenomena, 47, 219-230, 1991.

[22] C. S. Peskin, "Numerical Analysis of Blood Flow in the Heart". Journal of Computational Physics, 25, 220-252, 1977.

[23] Z. G. Feng, E. E. Michaelides, "The Immersed Boundary-Lattice Boltzmann Method for Solving Fluid-Particles Interaction Problems”. Journal of Computational Physics, 195, 602-628, 2004.

[24] Z. G. Feng, E. E. Michaelides, "Proteus: A Direct Forcing Method in the Simulations of Particulate Flows”. Journal of Computational Physics, 202, 20-51, 2005.

[25] E. A. Fadlun, R. Verzicco, P. Orlandi, J. Mohd. Yusof, "Combined Immersed Boundary Finite- Difference Methods for 3-D Complex Flow Simulations". Journal of Computational Physics, 161, 35-60, 2001.

[26] X. D. Niu, C. Shu, Y. T. Chew, Y. Peng, "A Momentum Exchanged-Based Immersed Boundary-Lattice Boltzmann Method for Simulating Incompressible Viscous Flows”. Physics Letters A, 354, 173-182, 2006.

[27] J. Wu, C. Shu, Y. H. Zhang, "Simulation of Incompressible Viscous Flows Around Moving Objects by a Variant of Immersed Boundary-Lattice Boltzmann Method”. International Journal for Numerical Methods in Fluids, 62, 327-354, 2010.

[28] J. Wu, Y. L. Qiu, C. Shu, N. Zhao, X. Wang, “An Adaptive Immersed Boundary-Lattice Boltzmann Method for Simulating a Flapping Foil in Ground Effect". Computers \& Fluids, 106, 171-184, 2015. 
[29] J. Wu, C. Liu, S. C. Yang, N. Zhao, "Influence of a Flexible Tail on the Performance of a Foil Hovering near the Ground: Numerical investigation". European Journal of Mechanics-B/Fluids, 52, 85-96, 2015.

[30] F. B. Tian, H Luo, L. Zhu, J. C. Liao, X. Y. Lu, “An Efficient Immersed Boundary-Lattice Boltzmann Method for the Hydrodynamic Interaction of Elastic Filaments", Journal of Computational Physics, 230(19), 7266-7283, 2011.

[31] H. Zhang, Y. Tan, S. Shu, et al., "Numerical Investigation on the Role of Discrete Element Method in Combined LBM-IBM-DEM Modeling”. Computers \& Fluids, 94, 37-48, 2014.

[32] R. Huang, H. Wu, “An Immersed Boundary-Thermal Lattice Boltzmann Method for SolidLiquid Phase Change”. Journal of Computational Physics, 277, 305-319, 2014.

[33] R. P. Selvam, P. C. Millett, "Computer Modeling of Tornado Forces on Buildings". Wind and Structures, 6(3), 209-220, 2003.

[34] R. P. Selvam, P. C. Millett, "Large Eddy Simulation of the Tornado-Structure Interaction to Determine Structural Loadings". Wind and Structures, 8(1), 49-60, 2005.

[35] P. Gorecki, R. P. Selvam, "Rankine Combined Vortex Interaction with a Rectangular Prism”. International Journal of Computational Fluid Dynamics, 29(1), 120-132, 2015.

[36] X. Guo, R. Palanisamy, J. Cao, “A Two-Dimensional IB-LBM Framework Combined with Re-Tailored RCVM for Assessing the Rotation Intensity of a Tornadic Wind over a Building Configuration”, Engineering Structures, 131, 57-68, 2017.

[37] C. S. Peskin, “The Immersed Boundary Method". Acta Numerica, 11, 479-517, 2002.

[38] L. Zhu, G. He, S. Wang, et al., "An Immersed Boundary Method based on the Lattice Boltzmann Approach in Three Dimensions with Application”. Computers and Mathematics with Applications, 61(12), 3506-3518, 2011. 
[39] J. Wu, C. Shu, “An Immersed Boundary-Lattice Boltzmann Method for Simulating ThreeDimensional Incompressible Flows". Journal of Computational Physics, 229(13), 5022$5042,2010$.

[40] X. Guo, J. Yao, C. Zhong, J. Cao, “A Hybrid Adaptive-Gridding Immersed-Boundary Lattice Boltzmann Method for Viscous Flow Simulations". Applied Mathematics and Computations, 267, 529-553, 2015.

[41] X. Guo, J. Cao, "An IB-LBM Investigation into the Aerodynamic Coefficients in Relation to the Rotation Intensity of a Tornado-like Wind". Computers and Mathematics with Applications, accepted, in press, https://doi.org/10.1016/j.camwa.2016.07.016.

[42] J. Smagorinsky, "General Circulation Experiments with the Primitive Equations: I. The Basic Experiment”. Monthly Weather Review, 91(3), 99-164, 1963. 